\title{
Logistic Neumann problems with discontinuous coefficients
}

\author{
Kazuaki Taira \\ Dedicated to Professor Hiroki Tanabe on the occasion of his 90th birthday
}

Received: date / Accepted: date

\begin{abstract}
The purpose of this paper is for the first time to provide a careful and accessible exposition of the study of the existence of positive solutions of semilinear Neumann problems for diffusive logistic equations with discontinuous coefficients, which models population dynamics in environments with spatial heterogeneity. A biological interpretation of our main result is that when the environment has an impassable boundary and is on the average unfavorable, then high diffusion rates have the same effect (that is, the ultimate extinction of the population) as they always have when the boundary is deadly; but if the boundary is impassable and the environment is on the average neutral or favorable, then the population can persist, no matter what its rate of diffusion. The approach here is based on explicit representation formulas for the solutions of the Neumann problem and also on the $L^{p}$ boundedness of Calderón-Zygmund singular integral operators appearing in those representation formulas. That is why we consider the case where the space dimension is greater than 2. Moreover, we make use of an $L^{p}$ variant of an estimate for the Green operator of the Neumann problem introduced in the study of Feller semigroups.
\end{abstract}

Keywords Neumann problem · diffusive logistic equation · VMO function · indefinite weight function $\cdot$ singular integral $\cdot$ representation formula $\cdot$ bifurcation theory $\cdot$ the sub-super-solution method $\cdot$ positive solution

Mathematics Subject Classification (2010) 92D25 - 35R05 - 42B20 - 60J70

\section{Contents}

1 Introduction and main results . . . . . . . . . . . . . . . . . . . . . . . . . 2

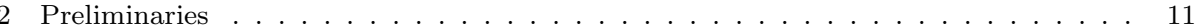

Proof of Theorem 1.1. . . . . . . . . . . . . . . . . . . . . . 16

Proof of parts (i) and (ii) of Theorem 1.2 . . . . . . . . . . . . . . . . . . . . . . 26

5 Proof of part (iii) of Theorem 1.2 . . . . . . . . . . . . . . . . . . . . . . 39

Kazuaki Taira

Institute of Mathematics, University of Tsukuba, Tsukuba 305-8571, Japan

E-mail: taira@math.tsukuba.ac.jp 
6 Existence and uniqueness theorems for semilinear eigenvalue problems . . . . . . 51

7 Proof of Theorem 1.3 . . . . . . . . . . . . . . . . . . . . 56

\section{Introduction and main results}

Let $\Omega$ be a bounded domain of $\mathbf{R}^{N}, N \geq 3$, with boundary $\partial \Omega$ of class $C^{1,1}$. In this paper we consider a second-order, uniformly elliptic differential operator with discontinuous coefficients in non-divergence form

$$
\mathcal{L} u:=-\sum_{i, j=1}^{N} a^{i j}(x) \frac{\partial^{2} u}{\partial x_{i} \partial x_{j}}+\sum_{i=1}^{N} b^{i}(x) \frac{\partial u}{\partial x_{i}} .
$$

Here:

(1) $a^{i j}(x) \in \mathrm{VMO} \cap L^{\infty}\left(\mathbf{R}^{N}\right), a^{i j}(x)=a^{j i}(x)$ for almost all $x \in \Omega$, and there exists a constant $a_{0}>0$ such that

$$
\frac{1}{a_{0}}|\xi|^{2} \leq \sum_{i, j=1}^{N} a^{i j}(x) \xi_{i} \xi_{j} \leq a_{0}|\xi|^{2} \quad \text { for almost all } x \in \Omega \text { and all } \xi \in \mathbf{R}^{N}
$$

(2) $b^{i}(x) \in L^{\infty}(\Omega)$ for $1 \leq i \leq N$.

In the case of continuous coefficients $a^{i j}(x)$, an $L^{p}$ Schauder theory has been elaborated for second-order, uniformly elliptic differential operators of the form (1.1) (see [4], [25]). However, the situation becomes rather difficult if we try to allow discontinuity on the $a^{i j}(x)$. In fact, it is known (see [36], [37], [57]) that arbitrary discontinuity of the $a^{i j}(x)$ breaks down as the $L^{p}$ Schauder theory, except for the two-dimensional case $(N=2)$. In order to handle with the case $N \geq 3$, additional conditions on the coefficients $a^{i j}(x)$ should be required. Here we assume that the coefficients $a^{i j}(x)$ belong to the Sarason class VMO of functions with vanishing mean oscillation. We recall that VMO consists of the John-Nirenberg class BMO of functions with bounded mean oscillation whose integral oscillation over balls shrinking to a point converge uniformly to zero (see Section 2).

This paper is devoted to the study of the existence of positive solutions of the following diffusive logistic Neumann problem with an indefinite weight and a positive parameter:

$$
\begin{cases}\mathcal{L} u=\lambda\left(m(x) u-h(x) u^{2}\right) & \text { in } \Omega \\ \frac{\partial u}{\partial \mathbf{n}}=0 & \text { on } \partial \Omega\end{cases}
$$

Here:

(3) $\lambda$ is a positive parameter.

(4) $m(x) \in C(\bar{\Omega})$ and $m(x)$ may change sign in $\Omega$.

(5) $h(x) \in C(\bar{\Omega})$ and $h(x) \geq 0$ on $\bar{\Omega}$.

(6) $\mathbf{n}$ is the unit outward normal to the boundary $\partial \Omega$. 
We discuss our motivation and some of the modeling process leading to the logistic Neumann problem (1.2) (see Tables 1.1 and 1.2). The basic interpretation of the various terms in the logistic Neumann problem (1.2) is that the solution $u(x)$ represents the population density of a species inhabiting the terrain $\Omega$. The members of the population are assumed to move about $\Omega$ via the type of random walks occurring in Markovian motion that is modeled by the diffusive term $(1 / \lambda) \mathcal{L}$; hence $1 / \lambda$ represents the rate of diffusive dispersal, so large values of $1 / \lambda$ the population spreads more rapidly than for small values of $1 / \lambda$. The local rate of change in the population density is described by the density dependent term $m(x)-$ $h(x) u$. In this term, $m(x)$ describes the rate at which the population would grow or decline at the location $x$ in the absence of crowding or limitations on the availability of resources. The sign of $m(x)$ will be positive on favorable habitats for population growth and negative on unfavorable ones. Specifically, $m(x)$ may be considered as a food source or any resource that will be good in some areas and bad in others. The term $-h(x) u$ describes the effects of crowding on the growth rate of the population at the location $x$; these effects are assumed to be independent of those determining the growth rate. The size of $h(x)$ describes the strength of the effects of crowding within the population.

On the other hand, in terms of biology, the homogeneous Dirichlet condition represents that $\Omega$ is surrounded by a completely hostile exterior such that any member of the population which reaches the boundary dies immediately; in other words, the exterior of the domain is deadly to the population. The logistic Dirichlet problem with VMO coefficients is studied in the previous paper [50] (see also [49] for $\mathcal{L}=-\Delta$ ).

If the boundary acts as a barrier, so that individuals reaching the boundary simply return to the interior, a Neumann boundary condition results (see [47], [52] for $\mathcal{L}=-\Delta$ ). However, the analysis is somewhat different from the Dirichlet case, since the operator $\mathcal{L}$ with homogeneous Neumann condition has zero as an eigenvalue (see [42], [12], [3] for $\mathcal{L}=-\Delta$ ).

The logistic Neumann problem may be treated just as in Senn [45] if we make use of the material of Senn and Hess [46], Maugeri and Palagachev [34] and Lieberman [33]. In this paper we introduce the closed realization $L$ of $\mathcal{L}$ with homogeneous Neumann condition in the Banach space $C(\bar{\Omega})$ as in Senn and Hess [46] and Senn [45]. However, because of discontinuity of the $a^{i j}(x)$ we re-work and expand in a different spirit the spectral analysis of the closed operator $L$ in the framework of the ordered Banach spaces $X=\mathcal{D}(L)$ and $Y=C(\bar{\Omega})$ adapted to the VMO case. In fact, we generalize Senn and Hess [46, Theorems 2 and 3] and Senn [45, Theorem 2.4] (Theorems 1.1, 1.2 and 1.3), by using a generation theorem for Feller semigroups with oblique derivative condition proved in the recent paper [56, Theorem $1.2]$.

The present paper is amply illustrated; 3 tables and 18 figures are provided with appropriate captions in such a fashion that a broad spectrum of readers could understand our problem and main results.

1.1 Neumann eigenvalue problems with indefinite weights

The first purpose of this paper is to generalize Senn-Hess [46, Theorems 2 and 3] and Senn [45, Theorem 2.4] to the VMO case. More precisely, we discuss the 


\begin{tabular}{|c|c|}
\hline Term & Biological interpretation \\
\hline \hline$\Omega$ & Terrain \\
\hline$u$ & Population density of a species inhabiting the terrain \\
\hline $\mathcal{L}$ & $\begin{array}{c}\text { A member of the population moves about the terrain } \\
\text { via the type of random walks occurring in Markovian motion }\end{array}$ \\
\hline $1 / \lambda$ & Rate of diffusive dispersal \\
\hline$m(x)$ & Intrinsic growth rate \\
\hline$h(x)$ & Coefficient of intraspecific competition \\
\hline
\end{tabular}

Table 1.1 A biological meaning of each term in the diffusive logistic Neumann problem (1.2)

\begin{tabular}{|c|c|}
\hline Boundary Condition & Biological interpretation \\
\hline \hline Dirichlet case & Completely hostile (deadly) exterior \\
\hline Neumann case & Barrier \\
\hline
\end{tabular}

Table 1.2 A biological meaning of boundary conditions

changes that occur in the global structure of positive solutions as a parameter $\lambda$ varies from the principal eigenvalue $\lambda_{1}(m)$ of the linearized homogeneous Neumann problem with an indefinite weight function

$$
\begin{cases}\mathcal{L} u=\lambda m(x) u & \text { in } \Omega, \\ \frac{\partial u}{\partial \mathbf{n}}=0 & \text { on } \partial \Omega .\end{cases}
$$

The next theorem plays an essential role in the study of the Neumann eigenvalue problem (1.3) with the indefinite weight function $m(x)$ (see [56, Theorem 1.2 and the Hille-Yosida theorem (Theorem 3.1)]):

Theorem 1.1 Let $N \geq 3$ and $N<p<\infty$. We define a linear operator

$$
L: C(\bar{\Omega}) \longrightarrow C(\bar{\Omega})
$$


as follows:

(a) The domain $\mathcal{D}(L)$ of definition is the set

$$
\mathcal{D}(L)=\left\{u \in W^{2, p}(\Omega): \mathcal{L} u \in C(\bar{\Omega}), \frac{\partial u}{\partial \mathbf{n}}=0 \text { on } \partial \Omega\right\} .
$$

(b) $L u=\mathcal{L} u$ for every $u \in \mathcal{D}(L)$.

Here $\mathcal{L} u$ and $\partial u / \partial \mathbf{n}$ are taken in the sense of distributions.

Then we have the following two assertions:

(i) The operator $L$ is densely defined and closed.

(ii) For any constant $\alpha>0$, the operator $L+\alpha I: \mathcal{D}(L) \rightarrow C(\bar{\Omega})$ is an algebraic and topological isomorphism, where the domain $\mathcal{D}(L)$ is equipped with the graph norm.

Remark 1.1 It is easy to verify that the domain $\mathcal{D}(L)$ is independent of $p$, for all $N<p<\infty$ (see [56, Section 9]).

\subsection{Logistic Neumann problems}

To study the logistic Neumann problem (1.2), we introduce two ordered Banach spaces and their positive cones associated with the operator $L$ in the following way: We work in the real Banach space

$$
Y:=C(\bar{\Omega})
$$

endowed with the maximum norm

$$
\|u\|_{Y}:=\max _{x \in \bar{\Omega}}|u(x)|,
$$

and provided with the natural ordering given by the positive cone

$$
P_{Y}:=\{v \in C(\bar{\Omega}): v \geq 0 \text { in } \Omega\} .
$$

Moreover, we use also the real Banach space

$$
X:=\mathcal{D}(L)=\left\{u \in W^{2, p}(\Omega): \mathcal{L} u \in C(\bar{\Omega}), \frac{\partial u}{\partial \mathbf{n}}=0 \text { on } \partial \Omega\right\} \quad \text { for } N<p<\infty
$$

endowed with the graph norm

$$
\|u\|_{X}=\|u\|_{Y}+\|L u\|_{Y}
$$

and provided with the natural ordering given by the positive cone

$$
\begin{aligned}
P_{X} & :=\{u \in \mathcal{D}(L): u \geq 0 \text { in } \Omega\} \\
& =\left\{u \in W^{2, p}(\Omega): \mathcal{L} u \in C(\bar{\Omega}), \frac{\partial u}{\partial \mathbf{n}}=0 \text { on } \partial \Omega, u \geq 0 \text { in } \Omega\right\} .
\end{aligned}
$$

Here it should be noticed that we have, by Sobolev's imbedding theorem (see [2, Theorem 5.4], [23, Part I, Theorem 10.2], [54, Theorems 4.17 and 4.19]),

$$
X=\mathcal{D}(L) \subset C^{1}(\bar{\Omega}),
$$


since $N<p<\infty$ and so $2-N / p>1$.

Moreover, it follows from an application of the Ascoli-Arzelà theorem (see [25, Lemma 6.36]) that the injection

$$
X \longrightarrow Y
$$

is compact.

The ordered Banach spaces $X$ and $Y$ and the operator $L$ can be visualized as in Figure 1.1.

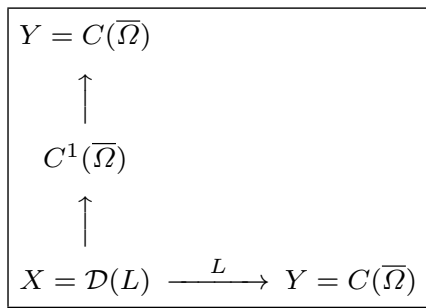

Fig. 1.1 The ordered Banach spaces $X$ and $Y$ and the operator $L$

Let $M: Y \rightarrow Y$ be the multiplication operator by a function $m(x) \in C(\bar{\Omega})$. A function $u \in X \backslash\{0\}$ is called an eigenfunction of the Neumann problem (1.3) if it satisfies the equation

$$
L u=\lambda M u \text { in } Y \text {. }
$$

We are interested in the existence of non-zero eigenvalues having a positive eigenfunction. It should be emphasized that if the weight function $m(x)$ does not change sign in $\Omega$, then there is no non-zero eigenvalue having a positive eigenfunction (see Claim 5.1 in Section 5). In this paper we study the logistic Neumann problem (1.2) under the condition that (see Remark 5.1 in Section 5):

(M) The weight function $m(x) \in C(\bar{\Omega})$ changes sign in $\Omega$, that is, it takes both positive and negative values in $\Omega$.

To formulate our results, we need the following proposition (see Figure 3.4 in Subsection 3.4):

Proposition 1.1 Since $1 \in \mathcal{N}(L)$, it follows from an application of the Krein-Rutman theorem (Theorem 2.1) that the Banach space adjoint operator

$$
L^{*}: Y^{*} \longrightarrow Y^{*}
$$

with domain $\mathcal{D}\left(L^{*}\right)$ has a one-dimensional null space $\mathcal{N}\left(L^{*}\right)$ spanned by an element $\psi \in Y^{*}$ with $\psi>0$. Moreover, we can identify $\psi$ with a positive function in $L^{q}(\Omega)$ for $q=p /(p-1)$, with $N<p<\infty$ :

$$
\begin{cases}\psi \in L^{q}(\Omega) & \text { for } q=p /(p-1), \\ L^{*} \psi=0 & \text { in } \Omega, \\ \psi>0 & \text { in } \Omega .\end{cases}
$$


Our first purpose is to generalize Senn-Hess [46, Theorems 2 and 3] to the VMO case:

Theorem 1.2 Let $N \geq 3$. Assume that condition (M) is satisfied. Then the Neumann eigenvalue problem (1.5) admits a unique non-zero, eigenvalue $\lambda_{1}(m)$ having a positive eigenfunction. More precisely, we have the following three assertions:

(i) If $\int_{\Omega} m(x) \psi(x) d x<0$, then the Neumann eigenvalue problem (1.5) admits a unique, positive eigenvalue $\lambda_{1}(m)$ with a positive eigenfunction in $\operatorname{Int}\left(P_{X}\right)$. Moreover, the eigenvalue $\lambda_{1}(m)$ has the following two properties:

(a) The eigenvalue $\lambda_{1}(m)$ is an $M$-simple eigenvalues of the operator $L$ (see conditions (H.1) and (H.2) below).

(b) If $\widehat{\lambda} \in \mathbf{C}$ is an eigenvalue of the equation

$$
\widehat{L} v=\widehat{\lambda} \widehat{M} v \quad \text { in } \widehat{Y} \text { for } \widehat{\lambda} \in \mathbf{C}
$$

obtained by the complexification of the operator equation (1.5) and if $\operatorname{Re} \widehat{\lambda}>0$, then it follows that

$$
\operatorname{Re} \widehat{\lambda} \geq \lambda_{1}(m) .
$$

The eigenvalue 0 is $M$-simple and has the positive eigenfunction $\phi_{1}(x) \equiv 1$ in $\Omega$.

(ii) If $\int_{\Omega} m(x) \psi(x) d x>0$, then the Neumann eigenvalue problem (1.5) admits a unique, negative eigenvalue $\lambda_{1}(m)$ with a positive eigenfunction in $\operatorname{Int}\left(P_{X}\right)$. Moreover, the eigenvalue $\lambda_{1}(m)$ has the following two properties:

(c) The eigenvalue $\lambda_{1}(m)$ and 0 are $M$-simple eigenvalues of the operator $L$.

(d) If $\widehat{\lambda} \in \mathbf{C}$ is an eigenvalue of the equation (1.7) in the complexification $\widehat{Y}$ of $Y$ and if $\operatorname{Re} \widehat{\lambda}<0$, then it follows that

$$
\operatorname{Re} \widehat{\lambda} \leq \lambda_{1}(m) .
$$

The eigenvalue 0 is $M$-simple and has the positive eigenfunction $\phi_{1}(x) \equiv 1$ in $\Omega$. (iii) If $\int_{\Omega} m(x) \psi(x) d x=0$, then the eigenvalue 0 of the Neumann eigenvalue problem $(1.5)$ is the only eigenvalue having the positive eigenfunction $\phi_{1}(x) \equiv 1$ in $\Omega$.

Here we recall that $\lambda \in \mathbf{R}$ is called an $M$-simple eigenvalue of $L$ if it satisfies the following two conditions:

(H.1) $\operatorname{dim} \mathcal{N}(L-\lambda M)=\operatorname{codim} \mathcal{R}(L-\lambda M)=1$.

(H.2) If $\mathcal{N}(L-\lambda M)=\operatorname{span}\left[u_{0}\right]$, then $M u_{0} \notin \mathcal{R}(L-\lambda M)$.

This notion is an extension of the concept of algebraic simplicity (see [20, Definition $1.2])$.

A pair $(\lambda, u) \in \mathbf{R} \times X$ is called a positive solution of the logistic Neumann problem (1.2) if $\lambda>0$ and $u \in P_{X} \backslash\{0\}$ and if the pair $(\lambda, u)$ satisfies the operator equation

$$
L u=\lambda F(u) \text { in } Y,
$$

where $F(u)$ is the Nemytskii operator associated with the nonlinear term $m(x) u-$ $h(x) u^{2}$ :

$$
F(u)(x)=m(x) u(x)-h(x) u(x)^{2} \quad \text { for } x \in \bar{\Omega} .
$$

Our second purpose is for the first time to prove the existence of positive solutions of the logistic Neumann problem (1.2) with VMO coefficients, generalizing Senn [45, Theorem 2.4]: 
Theorem 1.3 Let $N \geq 3$. Assume that condition ( $M)$ is satisfied and further that

$$
h(x)>0 \quad \text { on } \bar{\Omega} \text {. }
$$

Then we have the following four assertions:

(i) The case $\int_{\Omega} m(x) \psi(x) d x<0$ : There is an unbounded curve $\mathcal{C}$ of positive solutions $(\lambda, u(\lambda))$ of the logistic Neumann problem (1.2) emanating from the point $\left(\lambda_{1}(m), 0\right)$, with $\lambda>\lambda_{1}(m)$. Moreover, the point $\left(\lambda_{1}(m), 0\right)$ is the only bifurcation point for positive solutions from the line $\mathbf{R} \times\{0\}$ of trivial solutions (see Figure 1.2).

(ii) The case $\int_{\Omega} m(x) \psi(x) d x>0$ : There is an unbounded curve $\mathcal{C}$ of positive solutions $(\lambda, u(\lambda))$ of the logistic Neumann problem (1.2) emanating from the point $(0, c)$, with

$$
c=\frac{\int_{\Omega} m(x) \psi(x) d x}{\int_{\Omega} h(x) \psi(x) d x} .
$$

In other words, there is a secondary bifurcation from the line $\{0\} \times \mathbf{R}$ of trivial solutions, and non-constant solutions exist for arbitrarily small $\lambda>0$ (see Figure 1.3).

(iii) The case $\int_{\Omega} m(x) \psi(x) d x=0$ : There are two unbounded curves bifurcating at the point $(0,0)$ from the line $\mathbf{R} \times\{0\}$ of trivial solutions; namely, the line $\{0\} \times \mathbf{R}$ of trivial solutions and the positive solution curve $\mathcal{C}=\{(\lambda, u(\lambda)): \lambda>0\}$. In particular, non-constant solutions exist for arbitrarily small $\lambda>0$ (see Figure 1.4).

(iv) The positive solutions $u(\lambda)$ are uniformly bounded (see Figures 1.5 through 1.7):

$$
\max _{\bar{\Omega}}|u(\lambda)| \leq \frac{\max _{\bar{\Omega}} m}{\min _{\bar{\Omega}} h} .
$$

Remark 1.2 Some important remarks are in order:

$1^{\circ}$ Condition (1.9) is supposed to correspond to the competition for existence among living things in the terrain $\Omega$. See [51, Theorem 1.3] for $\mathcal{L}=-\Delta$.

$2^{\circ}$ By the uniform estimate (1.11), we find that the quantity

$$
\ell:=\frac{\max _{\bar{\Omega}} m}{\min \bar{\Omega} h}
$$

is the carrying capacity of the environment under the competition condition (1.9) (see Figures 1.5, 1.6 and 1.7). This idea is generally credited to the Belgian mathematical biologist P. F. Verhulst (1804-1849) around 1840.

Rephrased, Theorem 1.3 asserts that, in the case where $\int_{\Omega} m(x) \psi(x) d x<0$, the models we consider predict persistence for a population if its diffusion rate $1 / \lambda$ is below the critical value $1 / \lambda_{1}(m)$ depending on the coefficient $m(x)$ which describes the growth rate. On the other hand, if $\int_{\Omega} m(x) \psi(x) d x \geq 0$, then the models predict persistence for a population for any diffusion rate $1 / \lambda$.

A biological interpretation of our main result (Theorem 1.3) is that when the environment has an impassable boundary and is on the average unfavorable, then high diffusion rates have the same effect (that is, the ultimate extinction of the population) as they always have when the boundary is deadly (see [50, Theorem 1.3]); but if the boundary is impassable and the environment is on the average neutral or favorable, then the population can persist, no matter what its rate of diffusion. Our situation may be represented schematically by the six bifurcation diagrams, Figures 1.2 through 1.7. 


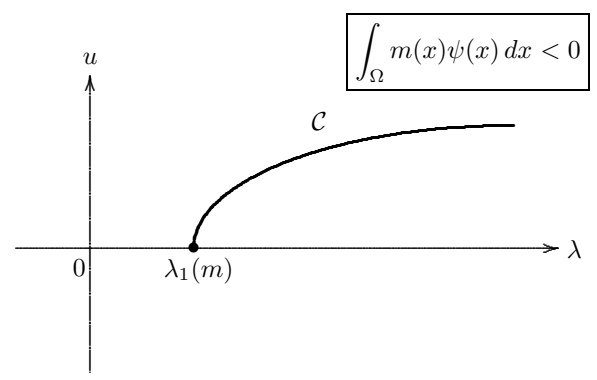

Fig. 1.2 A biological interpretation of part (i) of Theorem 1.3

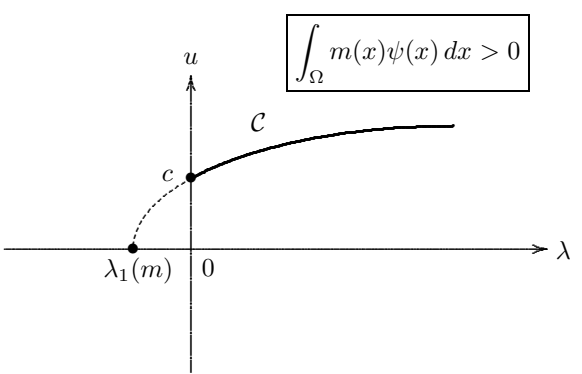

Fig. 1.3 A biological interpretation of part (ii) of Theorem 1.3

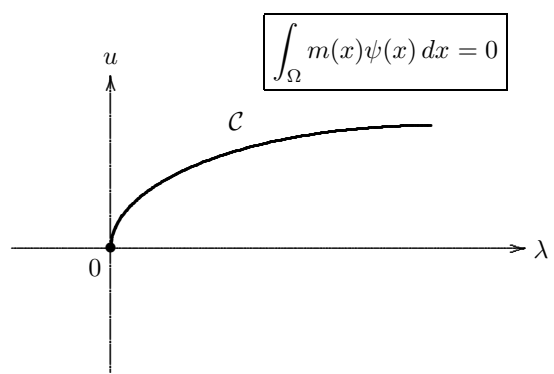

Fig. 1.4 A biological interpretation of part (iii) of Theorem 1.3

\subsection{An outline of the paper}

Our approach here is based on the Calderón-Zygmund theory of singular integral operators with non-smooth (i. e., non-infinitely differentiable) kernels. It should be emphasized that singular integral operators with non-smooth kernels provide a tool to deal with smoothness of solutions of partial differential equations, with minimal assumptions of regularity on the coefficients such as Miranda [37] and Talenti [57]. The theory of singular integrals continues to be one of the most influential works in modern history of analysis ([14], [58, Chapter 2]). Several recent developments in the theory of singular integrals have made possible further progress in the study of elliptic boundary value problems with discontinuous coefficients and hence in 


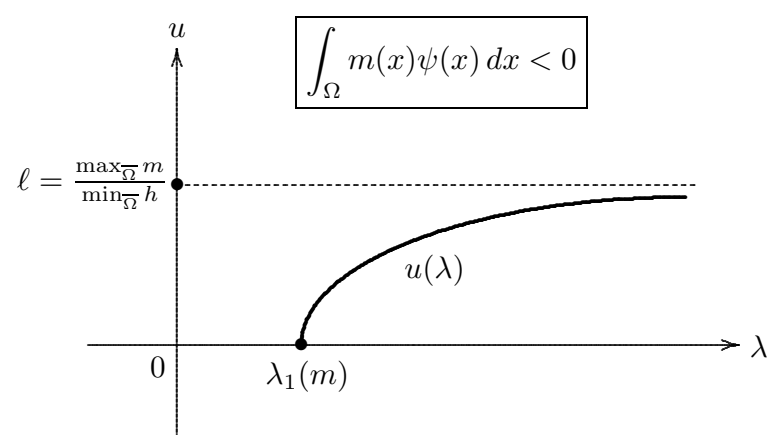

Fig. 1.5 A biological interpretation of part (iv) of Theorem 1.3 under condition (1.9)

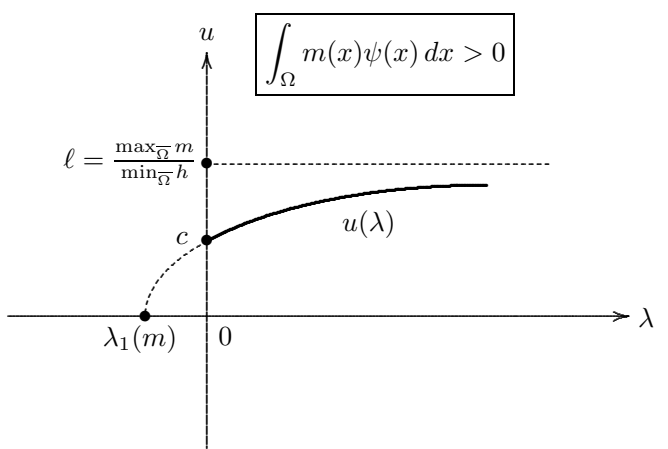

Fig. 1.6 A biological interpretation of part (iv) of Theorem 1.3 under condition (1.9)

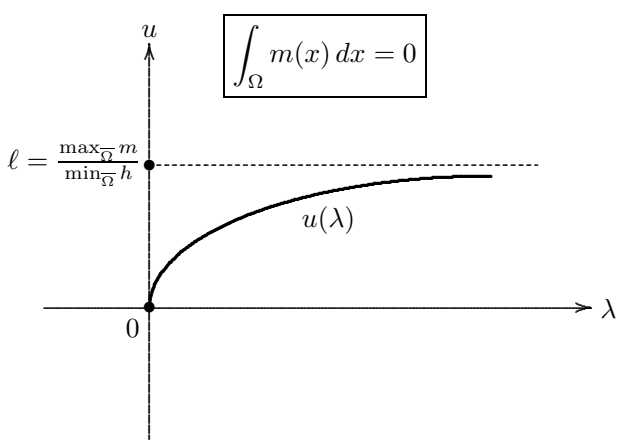

Fig. 1.7 A biological interpretation of part (iv) of Theorem 1.3 under condition (1.9)

the study of Markov processes. The presentation of these new results is the main purpose of this paper.

The rest of this paper is organized as follows.

In Section 2 we summarize some important topics from real analysis and nonlinear analysis such as VMO functions (Proposition 2.1), the KreĬn-Rutman theorem (Theorem 2.1) and local static bifurcation theory from a simple eigenvalue (The- 
orem 2.2). These topics form a necessary background for the proof of Theorems $1.1,1.2$ and 1.3 .

Section 3 is devoted to the proof of Theorem 1.1 and Proposition 1.1. We make essential use of an existence and uniqueness theorem for the Neumann problem with VMO coefficients (Theorem 3.2), which is proved in the pioneering papers Chiarenza-Frasca-Longo [16] and [17] by using the ideas and techniques characteristic of the theory of singular integral operators, and then extensively applied in the papers [11], [39] and [40]. It should be emphasized that the uniqueness result follows from an application of the Bakel'man-Aleksandrov maximum principle (Theorem 3.3). Moreover, the proof of the density of the domain $\mathcal{D}(L)$ is based on an $L^{p}$ variant of an estimate for the Green operator of the Neumann problem proved in the papers [53], [55] and [56] in the study of Feller semigroups (see assertion (3.6) and estimate (3.9)).

In Section 4 the proof of parts (i) and (ii) of Theorem 1.2 may be carried out just as in Senn-Hess [46] by using Theorem 1.1 and the Krein-Rutman theorem (Theorem 2.1). Compared with the Dirichlet case, the difficulty in the proof lies in the fact that the operator $L$ is not invertible. This section is the heart of the subject.

The proof of part (iii) of Theorem 1.2 is given in Section 5 due to its length. The essential step in the proof is Theorem 5.1, similar to Theorem 4.1, just as in Senn-Hess [46, Theorem 3].

In Section 6 we consider a general class of semilinear Neumann eigenvalue problems. By applying the super-subsolution method just as in [48], we prove existence and uniqueness theorems of positive solutions (Theorems 6.1 and 6.2). The proof of the existence result is based on Schauder's fixed point theorem (Theorem 6.3), and the proof of the uniqueness result is based on a uniqueness theorem of fixed points of strongly increasing and strongly sublinear mappings in ordered Banach spaces (Theorem 6.4).

The last Section 7 is devoted to the proof of Theorem 1.3. The proof of Theorem 1.3 may be carried out by constructing explicitly supersolutions and subsolutions to the logistic Neumann problem (1.2) (the sub-super-solution method).

Finally, for existence theorems of positive solutions for diffusive logistic equations with boundary conditions we give an overview of the classical Schauder theory versus the Calderón-Zygmund theory of singular integrals (see Table 1.3).

\section{Preliminaries}

This section is devoted to a review of some important topics from real analysis and nonlinear analysis that form a necessary background for the proofs of Theorems 1.1, 1.2 and 1.3. This makes the present paper fairly self-contained.

\subsection{The Spaces BMO and VMO}

In this subsection we recall some basic definitions and results concerning BMO and VMO functions on $\mathbf{R}^{N}$. For more thorough treatments of this subject, the reader might be referred to Garnett [24] and Torchinsky [59]. 


\begin{tabular}{|c|c|c|}
\hline $\begin{array}{l}\text { Diffusive logistic } \\
\text { equations }\end{array}$ & $\begin{array}{c}\text { Classical } \\
\text { Schauder theory }\end{array}$ & $\begin{array}{c}\text { Calderón-Zygmund } \\
\text { theory }\end{array}$ \\
\hline $\begin{array}{l}\text { Regularity of the } \\
\text { leading coefficients }\end{array}$ & $\begin{array}{l}\text { Hölder continuous } \\
\text { functions }\end{array}$ & VMO functions \\
\hline Dirichlet case & $\begin{array}{c}{[28, \text { Theorem 2] }} \\
{[27, \text { Theorem 27.1] }}\end{array}$ & [50, Theorem 1.3] \\
\hline Neumann case & {$[46$, Theorems 2 and 3$]$} & Theorem 1.2 \\
\hline
\end{tabular}

Table 1.3 An overview of the classical Schauder theory versus the Calderón-Zygmund theory of singular integrals for diffusive logistic equations in non-divergence form

A function $f(x) \in L_{\mathrm{loc}}^{1}\left(\mathbf{R}^{N}\right)$ is said to be of bounded mean oscillation, $f(x) \in$ $\mathrm{BMO}$, if it satisfies the condition (see [29])

$$
\|f\|_{*}:=\sup _{B} \frac{1}{|B|} \int_{B}\left|f(x)-f_{B}\right| d x<\infty
$$

where the supremum is taken over all balls $B$ in $\mathbf{R}^{N}$ and $f_{B}$ is the average of $f$ over $B$

$$
f_{B}:=\frac{1}{|B|} \int_{B} f(x) d x
$$

It should be noticed that the quantity $\|f\|_{*}$ defines a norm on the quotient space $\mathrm{BMO} / \mathbf{R}$.

Next we introduce a subspace of BMO functions whose BMO norm over a ball vanishes as the radius of the ball tends to zero. More precisely, if $f(x) \in \mathrm{BMO}$ and $r>0$, then we let

$$
\eta(r):=\sup _{\rho \leq r} \frac{1}{|B|} \int_{B}\left|f(x)-f_{B}\right| d x
$$

where the supremum is taken over all balls $B$ with radius $\rho \leq r$.

A function $f(x) \in \mathrm{BMO}$ has vanishing mean oscillation, $f(x) \in \mathrm{VMO}$, if it satisfies the condition (see [41])

$$
\lim _{r \downarrow 0} \eta(r)=0 .
$$

The function $\eta(r)$ is called the VMO modulus of $f$.

The assumption $a^{i j}(x) \in \mathrm{VMO}$ means a kind of continuity in the average sense, not in the pointwise sense. This property implies that VMO functions may be approximated by smooth functions.

The relationship between $\mathrm{BMO}$ and its subspace VMO is quite similar to the relationship between $L^{\infty}$ and its subspace BUC of bounded uniformly continuous functions (see Figure 2.1).

The next proposition collects some important results concerning VMO functions (see [24, Chapter VI, Theorem 5.1], [59, Chapter VIII]): 


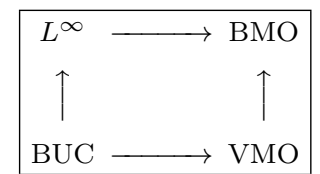

Fig. 2.1 The spaces $L^{\infty}$, BMO, BUC and VMO

Proposition 2.1 (i) If $f(x) \in \mathrm{VMO}$, then, for any $\varepsilon>0$ there exists a uniformly continuous function $g_{\varepsilon}(x)$ on $\mathbf{R}^{N}$ such that $\left\|f-g_{\varepsilon}\right\|_{*}<\varepsilon$.

(ii) Uniformly continuous functions that belong to BMO are VMO functions.

(iii) $\mathrm{VMO}$ is a closed subspace of $\mathrm{BMO}$. 1.3]).

(iv) VMO functions are invariant under $C^{1,1}$-diffeomorphisms ([1, Proposition

(v) For example, $W^{\theta, N / \theta}\left(\mathbf{R}^{N}\right) \subset \mathrm{VMO}$ for all $0<\theta \leq 1$.

\subsection{The KreĬn and Rutman theorem}

In this subsection we recall some basic definitions and results concerning ordered Banach spaces. For more thorough treatments of this subject, the reader might be referred to Amann [5], Brown [13, Chapter 18], Chang [15, Chapter 3] and Drábek and Milota [22, Chapter 6].

Let $X$ be a non-empty set. An ordering $\leq$ in $X$ is a relation in $X$ that is reflexive, transitive and antisymmetric. A non-empty set together with an ordering is called an ordered set.

Let $V$ be a real vector space. An ordering $\leq$ in $V$ is said to be linear if the following two conditions are satisfied:

(i) If $x, y \in V$ and $x \leq y$, then we have $x+z \leq y+z$ for all $z \in V$.

(ii) If $x, y \in V$ and $x \leq y$, then we have $\alpha x \leq \alpha y$ for all $\alpha \geq 0$.

A real vector space together with a linear ordering is called an ordered vector space.

If $x, y \in V$ and $x \leq y$, then the set $[x, y]=\{z \in X: x \leq z \leq y\}$ is called an order interval.

If we let

$$
Q=\{x \in V: x \geq 0\},
$$

then it is easy to verify that the set $Q$ satisfies the following two conditions:

(iii) If $x, y \in Q$, then $\alpha x+\beta y \in Q$ for all $\alpha, \beta \geq 0$.

(iv) If $x \neq 0$, then at least one of $x$ and $-x$ does not belong to $Q$, or equivalently, $Q \cap(-Q)=\{0\}$.

The set $Q$ is called the positive cone of the ordering $\leq$.

Let $E$ be a Banach space $E$ with a linear ordering $\leq$. The Banach space $E$ is called an ordered Banach space if the positive cone $P$ is closed in $E$. It is to be expected that the topology and the ordering of an ordered Banach space are closely related if the norm is monotone: If $0 \leq x \leq y$, then $\|x\| \leq\|y\|$.

For $x, y \in E$, we write

$$
x \geq y \quad \text { if } x-y \in P,
$$




$$
x>y \quad \text { if } x-y \in P \backslash\{0\} .
$$

If the interior $\operatorname{Int}(P)$ is non-empty, then we write

$$
x \gg y \quad \text { if } x-y \in \operatorname{Int}(P) \text {. }
$$

Example 2.1 Let $Y:=C(\bar{\Omega})$. For two functions $u, v \in Y$, we write $u \leq v$ if $u(x) \leq$ $v(x)$ for all $x \in \bar{\Omega}$. Then it is easy to verify that the space $Y$ is an ordered Banach space with the linear ordering $\leq$ and the positive cone

$$
P_{Y}=\{u \in C(\bar{\Omega}): u \geq 0 \text { on } \bar{\Omega}\},
$$

with non-empty interior

$$
\operatorname{Int}\left(P_{Y}\right)=\{u \in C(\bar{\Omega}): u>0 \text { on } \bar{\Omega}\} .
$$

A linear operator $A: E \rightarrow E$ is said to be strongly positive if $A x$ is an interior point of $P$ for every $x \in P \backslash\{0\}$ :

$$
x>0 \Longrightarrow A x \gg 0 .
$$

Then the Kreĭn and Rutman theorem reads as follows (see KreĬn and Rutman [32, Theorem 6.3]):

Theorem 2.1 (Kreŭn-Rutman) Let $(E, P)$ be an ordered Banach space with nonempty interior Int $(P)$. Assume that

$$
K: E \longrightarrow E
$$

is a strongly positive and compact operator. Then we have the following three assertions:

(i) The spectral radius

$$
r=\operatorname{spr}(K):=\lim _{n \rightarrow \infty}\left\|K^{n}\right\|^{1 / n}
$$

is positive, and $r$ is a unique eigenvalue of $K$ having positive eigenfunction $x \in$ Int $(P)$. The eigenvalue $r$ is algebraically simple.

(ii) Moreover, $r$ is also an algebraically simple eigenvalue of the adjoint operator

$$
K^{*}: E^{*} \longrightarrow E^{*}
$$

with positive eigenfunction $x^{*} \in \operatorname{Int}\left(P^{*}\right)$. Here

$$
P^{*}=\left\{x^{*} \in E^{*}:\left\langle x^{*}, x\right\rangle \geq 0 \text { for all } x \in P\right\} .
$$

(iii) Finally, we have $|\lambda|<r$ for all $\lambda \in \sigma(K)$ with $\lambda \neq r$, where $\sigma(K)$ is the spectrum of $K$.

The eigenvalue $r$ is called the principal eigenvalue of $K$. 


\subsection{Local bifurcation theory}

This subsection is devoted to local static bifurcation theory from a simple eigenvalue essentially due to Crandall and Rabinowitz [19]. For detailed studies of bifurcation theory, the reader is referred to Ambrosetti and Malchiodi [7], Ambrosetti and Prodi [8], Brown [13], Chang [15], Chow and Hale [18], Drábek and Milota [22] and Nirenberg [38].

Let $F(t, x)$ be a mapping of a neighborhood of $(0,0)$ in a Banach space $\mathbf{R} \times X$ into a Banach space $Y$. Assume that there is a curve $\Gamma$ in the space $\mathbf{R} \times X$ given by $\Gamma=\{w(t): t \in I\}$, where $I$ is an interval, such that $F(w)=0$ for all $w \in \Gamma$. If there is a number $\tau_{0} \in I$ such that every neighborhood of $w\left(\tau_{0}\right)$ contains zeros of $F$ not lying on $\Gamma$, then the point $w\left(\tau_{0}\right)$ is called a bifurcation point for the equation $F(w)=0$ with respect to the curve $\Gamma$. In many situations the curve $\Gamma$ is of the form $\{(t, 0): t \in \mathbf{R}, 0 \in X\}$. The basic problem of bifurcation theory is that of finding the bifurcation points for the equation $F(t, x)=0$ with respect to $\Gamma$ and studying the structure of $F^{-1}\{0\}$ near such points.

The next theorem, due to Crandall and Rabinowitz [19], gives sufficient conditions in order that the point $(0,0)$ is a bifurcation point for the equation $F(t, x)=0$ (see [19, Theorem 1.7]):

Theorem 2.2 (Crandall-Rabinowitz) Let $X, Y$ be Banach spaces, and let $V$ be a neighborhood of 0 in $X$. Assume that the map

$$
F:(-1,1) \times V \longrightarrow Y
$$

enjoys the following four properties:

(1) $F(t, 0)=0$ for $|t|<1$.

(2) The partial Fréchet derivatives $F_{t}, F_{x}$ and $F_{t x}$ of $F$ exist and are continuous.

(3) $\operatorname{dim} \mathcal{N}\left(F_{x}(0,0)\right)=\operatorname{codim} \mathcal{R}\left(F_{x}(0,0)\right)=1$.

(4) If $\mathcal{N}\left(F_{x}(0,0)\right)=\operatorname{span}\left[x_{0}\right]$, then $F_{t x}(0,0) x_{0} \notin \mathcal{R}\left(F_{x}(0,0)\right)$.

If $Z$ is a complement of $\mathcal{N}\left(F_{x}(0,0)\right)$ in $X$, that is, if it is a closed subspace of $X$ such that

$$
X=\mathcal{N}\left(F_{x}(0,0)\right) \oplus Z,
$$

then there exist a neighborhood $U$ of the point $(0,0)$ in $\mathbf{R} \times X$ and an open interval $(-a, a)$ such that the set of solutions of the nonlinear operator equation $F(t, x)=0$ in $U$ consists precisely of two continuous curves $\Gamma_{1}$ and $\Gamma_{2}$ which may be parametrized by $t$ and $\alpha$ as follows:

$$
\begin{aligned}
& \Gamma_{1}=\{(t, 0):(t, 0) \in U\}, \\
& \Gamma_{2}=\left\{\left(\varphi(\alpha), \alpha x_{0}+\alpha \psi(\alpha)\right):|\alpha|<a\right\} .
\end{aligned}
$$

Here

$$
\begin{aligned}
& \varphi:(-a, a) \longrightarrow \mathbf{R}, \quad \varphi(0)=0, \\
& \psi:(-a, a) \longrightarrow Z, \quad \psi(0)=0 .
\end{aligned}
$$

If, in addition, the partial Fréchet derivative $F_{x x}$ is also continuous, then the functions $\varphi$ and $\psi$ are once continuously differentiable. 


\section{Proof of Theorem 1.1}

This section is devoted to the proof of Theorem 1.1. Our proof is based on an existence and uniqueness theorem for the Neumann problem with VMO coefficients. To prove the density of the domain $\mathcal{D}(L)$, we make use of an $L^{p}$ variant of an estimate for the Green operator of the Neumann problem proved by the recent paper [56, Theorem 1.2] in the study of Feller semigroups (see assertion (3.6) and estimate (3.9)).

\subsection{The Neumann problem}

In this subsection we consider the Neumann problem in the framework of Sobolev spaces of $L^{p}$ style.

If $1<p<\infty$ and $k$ is a positive integer, we define the Sobolev space

$$
\begin{aligned}
W^{k, p}(\Omega)= & \text { the space of (equivalence classes of) functions } \\
& u \in L^{p}(\Omega) \text { whose derivatives } D^{\alpha} u,|\alpha| \leq k, \text { in the } \\
& \text { sense of distributions are in } L^{p}(\Omega) .
\end{aligned}
$$

If $1<p<\infty$, we define the boundary space

$$
\begin{aligned}
B^{1-1 / p, p}(\partial \Omega)= & \text { the space of the boundary values }\left.u\right|_{\partial \Omega} \\
& \text { of functions } u \in W^{1, p}(\Omega),
\end{aligned}
$$

with a norm

$$
|\varphi|_{B^{1-1 / p, p}(\partial \Omega)}=\inf \left\{\|u\|_{W^{1, p}(\Omega)}: u \in W^{1, p}(\Omega),\left.u\right|_{\partial \Omega}=\varphi\right\} .
$$

More precisely, the space $B^{1-1 / p, p}(\partial \Omega)$ is a Besov space (see [2], [9], [60]).

Our starting point is the following existence and uniqueness theorem for the Neumann problem with VMO coefficients (see [34, Theorem 4.1], [35, Theorem 2.3.5], [56, Theorem 5.1]):

Theorem 3.1 Let $N<p<\infty$ and $\alpha>0$. Then the non-homogeneous Neumann problem

$$
\begin{cases}(\mathcal{L}+\alpha) u=f & \text { in } \Omega, \\ B u=\varphi & \text { on } \partial \Omega\end{cases}
$$

has a unique solution $u \in W^{2, p}(\Omega)$ for any $f \in L^{p}(\Omega)$ and any $\varphi \in B^{1-1 / p, p}(\partial \Omega)$. Here

$$
B u:=\frac{\partial u}{\partial \mathbf{n}} .
$$

Moreover, we have the a priori estimate

$$
\|u\|_{W^{2, p}(\Omega)} \leq C\left(\|(\mathcal{L}+\alpha) u\|_{L^{p}(\Omega)}+|B u|_{B^{1-1 / p, p}(\partial \Omega)}\right),
$$

with a constant $C>0$, independent of $u$. 
If we associate with the Neumann problem (3.1) a continuous linear operator

$$
\mathcal{A}(\alpha)=(\mathcal{L}+\alpha, B): W^{2, p}(\Omega) \longrightarrow L^{p}(\Omega) \oplus B^{1-1 / p, p}(\partial \Omega)
$$

then Theorem 3.1 asserts that the mapping $\mathcal{A}(\alpha)$ is an algebraic and topological isomorphism with the a priori estimate (3.2). Indeed, the continuity of the inverse of $\mathcal{A}$ follows immediately from an application of Banach's closed graph theorem (see [43, Theorem 3.10], [62, Chapter II, Section 6, Theorem 1]).

\subsection{Proof of Theorem 3.1}

Step 1: Our proof is based on the following existence and uniqueness theorem for the homogeneous Neumann problem due to Di Fazio and Palagachev [21]:

Theorem 3.2 Let $1<p<\infty$ and

$$
\mathcal{L}_{0} u:=-\sum_{i, j=1}^{N} a^{i j}(x) \frac{\partial^{2} u}{\partial x_{i} \partial x_{j}} .
$$

Then there exists a constant $\alpha_{0}>0$ such that the homogeneous Neumann problem

$$
\begin{cases}\left(\mathcal{L}_{0}+\alpha_{0}\right) u=f & \text { in } \Omega, \\ B u=0 & \text { on } \partial \Omega\end{cases}
$$

has a unique solution $u \in W^{2, p}(\Omega)$ for any $f \in L^{p}(\Omega)$.

The proof of Theorem 3.2 is given in detail in Di Fazio and Palagachev [21, Theorem 1.2 and Remark 1.1] (see also Maugeri-Palagachev-Softova [35, pp. 138139]).

(1) The proof is based on some interior and boundary estimates for the solutions of the Neumann problem 3.3. From these estimates, an a priori estimate follows. Since VMO functions can be approximated by smooth functions, we can prove the existence result of the Neumann problem (3.3) in a standard way if we approximate the operator $\mathcal{L}_{0}$ with similar operators with smooth coefficients. Both the interior and boundary estimates are consequences of explicit representation formulas for the solutions of the Neumann problem (3.3) (see [21, Lemma 3.1]) and also of the $L^{p}$-boundedness of Calderón-Zygmund singular integral operators appearing in those representation formulas (see [21, Lemma 4.2]).

(2) For the uniqueness result of the Neumann problem (3.3), we make essential use of the Bakel'man and Aleksandrov maximum principle that requires the VMO assumption on the coefficients.

Now, for any $\varphi \in B^{1-1 / p, p}(\partial \Omega)$ we can find a function $v \in W^{2, p}(\Omega)$ such that $B v=\varphi$. Hence we have the following existence and uniqueness theorem for the non-homogeneous Neumann problem:

Corollary 3.1 Let $1<p<\infty$. For any $f \in L^{p}(\Omega)$ and any $\varphi \in B^{1-1 / p, p}(\partial \Omega)$, there exists a unique solution $u \in W^{2, p}(\Omega)$ of the non-homogeneous Neumann problem

$$
\begin{cases}\left(\mathcal{L}_{0}+\alpha_{0}\right) u=f & \text { in } \Omega, \\ B u=\varphi & \text { on } \partial \Omega .\end{cases}
$$


If we associate with the Neumann problem (3.4) a continuous linear operator

$$
\mathcal{A}\left(\alpha_{0}\right)=\left(\mathcal{L}_{0}+\alpha_{0}, B\right): W^{2, p}(\Omega) \longrightarrow L^{p}(\Omega) \oplus B^{1-1 / p, p}(\partial \Omega),
$$

then Corollary 3.1 asserts that the mapping $\mathcal{A}\left(\alpha_{0}\right)$ is an algebraic and topological isomorphism. In particular, we have the formula

$$
\text { ind } \mathcal{A}\left(\alpha_{0}\right)=0 \text {. }
$$

Step 2: If we let

$$
\mathcal{B}\left(\alpha-\alpha_{0}\right) u:=\sum_{i=1}^{N} b^{i}(x) \frac{\partial u}{\partial x_{i}}+\left(\alpha-\alpha_{0}\right) u,
$$

then it is clear that the operator

$$
\mathcal{B}\left(\alpha-\alpha_{0}\right): W^{2, p}(\Omega) \longrightarrow W^{1, p}(\Omega)
$$

is continuous. Moreover, it follows from an application of the Rellich-Kondrachov theorem (see [2, Theorem 6.3], Friedman [23, Part I, Theorem 11.2], [25, Section 7.12, Theorem 7.26]) that the injection

$$
W^{1, p}(\Omega) \longrightarrow L^{p}(\Omega) \quad \text { for } 1<p<\infty
$$

is compact. Hence, we find that the mapping

$$
\mathcal{B}\left(\alpha-\alpha_{0}\right): W^{2, p}(\Omega) \longrightarrow L^{p}(\Omega)
$$

is compact for $1<p<\infty$. It should be noticed that

$$
\mathcal{A}(\alpha)=(\mathcal{L}+\alpha, B)=\left(\mathcal{L}_{0}+\alpha_{0}, B\right)+\left(\mathcal{B}\left(\alpha-\alpha_{0}\right), 0\right)=\mathcal{A}\left(\alpha_{0}\right)+\left(\mathcal{B}\left(\alpha-\alpha_{0}\right), 0\right) .
$$

However, we know (see [26, Theorem 2.6], [43, Theorem 5.10]) that the index is stable under compact perturbations.

Therefore, we obtain that the mapping

$$
\mathcal{A}(\alpha)=\mathcal{A}\left(\alpha_{0}\right)+\left(\mathcal{B}\left(\alpha-\alpha_{0}\right), 0\right): W^{2, p}(\Omega) \longrightarrow L^{p}(\Omega) \oplus B^{1-1 / p, p}(\partial \Omega)
$$

is a Fredholm operator with index zero, since we have, by formula (3.5),

$$
\text { ind } \mathcal{A}(\alpha)=\text { ind } \mathcal{A}\left(\alpha_{0}\right)=0 \text { for } 1<p<\infty \text {. }
$$

Step 3: On the other hand, the uniqueness result in Theorem 3.1 follows from an application of the Bakel'man and Aleksandrov maximum principle (see [10, Théorème 2]; [25, Theorem 9.1]; [33, Corollary 2.5]; [61, p. 187, Section 3.7]):

Theorem 3.3 (the strong maximum principle) Let $\alpha>0$ and assume that

$$
\left\{\begin{array}{l}
u \in C^{1}(\bar{\Omega}) \cap W_{\text {loc }}^{2, N}(\Omega), \\
(\mathcal{L}+\alpha) u \geq 0 \quad \text { almost everywhere in } \Omega, \\
B u \geq 0 \quad \text { on } \partial \Omega, \\
\min _{\bar{\Omega}} u \leq 0 .
\end{array}\right.
$$

Then it follows that $u(x)=0$ in $\Omega$. 
However, we have, by Sobolev's imbedding theorem (see [2, Theorem 5.4], [23, Part I, Theorem 10.2], [54, Theorems 4.17 and 4.19]),

$$
W^{2, p}(\Omega) \subset C^{1}(\bar{\Omega}) \cap W^{2, N}(\Omega) \text { for } N<p<\infty,
$$

since $2-N / p>1$.

Therefore, by applying Theorem 3.3 to the functions $\pm u(x)$ we find that

$$
\left\{\begin{array}{ll}
(\mathcal{L}+\alpha) u=0 & \text { almost everywhere in } \Omega, \Longrightarrow u=0 \\
B u=0 & \text { on } \partial \Omega
\end{array} \text { in } \Omega .\right.
$$

This proves that the mapping

$$
\mathcal{A}(\alpha)=(\mathcal{L}+\alpha, B): W^{2, p}(\Omega) \longrightarrow L^{p}(\Omega) \oplus B^{1-1 / p, p}(\partial \Omega)
$$

is injective for $N<p<\infty$. Hence it is also surjective for $N<p<\infty$, since we have the assertion

$$
\text { ind } \mathcal{A}(\alpha)=0 \text {. }
$$

Step 4: Summing up, we have proved that the mapping

$$
\mathcal{A}(\alpha)=(\mathcal{L}+\alpha, B): W^{2, p}(\Omega) \longrightarrow L^{p}(\Omega) \oplus B^{1-1 / p, p}(\partial \Omega)
$$

is an algebraic and topological isomorphism for $N<p<\infty$.

The proof of Theorem 3.1 is complete.

\subsection{Proof of Theorem 1.1}

The proof is divided into five steps.

Step 1: First, we prove that, for each $\alpha>0$, the equation $(\alpha I+L) u=f$ has a unique solution $u \in \mathcal{D}(L)$ for any $f \in C(\bar{\Omega})$.

By applying Theorem 3.1, we obtain that the Neumann problem

$$
\begin{cases}(\alpha+\mathcal{L}) u=f & \text { almost everywhere in } \Omega \\ \frac{\partial u}{\partial \mathbf{n}}=0 & \text { on } \partial \Omega\end{cases}
$$

has a unique solution $u \in W^{2, p}(\Omega)$ for any $f \in L^{p}(\Omega)$ with $N<p<\infty$. In particular, for any $f \in C(\bar{\Omega})$ there exists a function $u \in W^{2, p}(\Omega)$ such that

$$
(\alpha+\mathcal{L}) u=f \quad \text { in } \Omega .
$$

Hence, we have the assertion

$$
\mathcal{L} u=f-\alpha u \in C(\bar{\Omega}) .
$$

This proves that

$$
\left\{\begin{array}{l}
u \in \mathcal{D}(L) \\
(\alpha I+L) u=f .
\end{array}\right.
$$

Step 2: Secondly, we prove that, for each $\alpha>0$, the Green operator

$$
G_{\alpha}^{N}=(\alpha I+L)^{-1}: C(\bar{\Omega}) \longrightarrow C(\bar{\Omega})
$$


is non-negative (see [56, Theorem 1.2]):

$$
f \in C(\bar{\Omega}), f(x) \geq 0 \quad \text { in } \Omega \Longrightarrow u(x)=G_{\alpha}^{N} f(x) \geq 0 \quad \text { in } \Omega .
$$

More precisely, we have the following assertion:

$$
f \in C(\bar{\Omega}), f(x) \geq 0, f(x) \not \equiv 0 \quad \text { in } \Omega \Longrightarrow G_{\alpha}^{N} f(x)>0 \text { on } \bar{\Omega} .
$$

In other words, the operator $G_{\alpha}^{N}: C(\bar{\Omega}) \rightarrow C(\bar{\Omega})$ is strongly positive.

If we let

$$
u(x):=G_{\alpha}^{N} f(x)
$$

then we obtain that

$$
\begin{cases}u \in W^{2, p}(\Omega) & \text { for } N<p<\infty \\ (\mathcal{L}+\alpha) u=f \geq 0 & \text { almost everywhere in } \Omega, \\ \frac{\partial u}{\partial \mathbf{n}}=0 & \text { on } \partial \Omega .\end{cases}
$$

It follows from an application of Theorem 3.3 that

$$
\min _{\bar{\Omega}} u \leq 0 \Longrightarrow u(x) \equiv 0 \quad \text { in } \Omega .
$$

This implies a contradiction that

$$
f(x)=(\mathcal{L}+\alpha) u(x) \equiv 0 \quad \text { in } \Omega .
$$

Therefore, we have proved that

$$
\min _{\bar{\Omega}} u>0
$$

that is,

$$
G_{\alpha}^{N} f(x)>0 \text { on } \bar{\Omega} .
$$

Step 3: Thirdly, we prove that, for each $\alpha>0$, the Green operator $G_{\alpha}^{N}=$ $(\alpha I+L)^{-1}$ is bounded on the space $C(\bar{\Omega})$ with norm $1 / \alpha$ :

$$
\left\|G_{\alpha}^{N}\right\| \leq \frac{1}{\alpha}
$$

By assertion (3.6), it suffices to show that

$$
\alpha G_{\alpha}^{N} 1(x) \leq 1 \quad \text { on } \bar{\Omega} .
$$

If we let

$$
v(x):=\alpha G_{\alpha}^{N} 1(x)-1,
$$

then we have the assertion

$$
\max _{\bar{\Omega}} v \leq 0
$$

that is,

$$
\alpha G_{\alpha}^{N} 1(x) \leq 1 \quad \text { on } \bar{\Omega} .
$$


Indeed, since we have the assertions

$$
\begin{cases}v \in W^{2, p}(\Omega) & \text { for } N<p<\infty \\ (\mathcal{L}+\alpha) v=0 & \text { in } \Omega \\ \frac{\partial v}{\partial \mathbf{n}}=0 & \text { on } \partial \Omega\end{cases}
$$

it follows from an application of Theorem 3.3 with $u(x):=-v(x)$ that

$$
\max _{\bar{\Omega}} v=-\min _{\bar{\Omega}} u>0 \Longrightarrow v(x)=-u(x) \equiv 0 \quad \text { in } \Omega .
$$

This contradiction proves that

$$
\max _{\bar{\Omega}} v \leq 0
$$

Step 4: The closedness of $L$ is an immediate consequence of the boundedness of $G_{\alpha}^{N}=(\alpha I+L)^{-1}$. Indeed, it suffices to note the formula

$$
L=\left(G_{\alpha}^{N}\right)^{-1}-\alpha I .
$$

Step 5: Finally, we prove that the domain $\mathcal{D}(L)$ is dense in $C(\bar{\Omega})$. More precisely, we prove that, for each $u \in C(\bar{\Omega})$, we have the assertion

$$
\lim _{\alpha \rightarrow+\infty}\left\|\alpha G_{\alpha}^{N} u-u\right\|_{C(\bar{\Omega})}=0
$$

It suffices to prove assertion (3.7) for all $v \in C^{2}(\bar{\Omega})$ such that

$$
\frac{\partial v}{\partial \mathbf{n}}=0 \quad \text { on } \partial \Omega
$$

In fact, we have the following density lemma in the space $C(\bar{\Omega})$ (see $[6$, Lemma 3.2], [53, Lemma 4.4]):

Lemma 3.1 Let $\varepsilon>0$ be arbitrary. For any given function $u \in C(\bar{\Omega})$, there exists a function $v \in C^{2}(\bar{\Omega})$ such that

$$
\left\{\begin{array}{l}
\|u-v\|_{C(\bar{\Omega})}<\varepsilon \\
\frac{\partial v}{\partial \mathbf{n}}=0 \quad \text { on } \partial \Omega
\end{array}\right.
$$

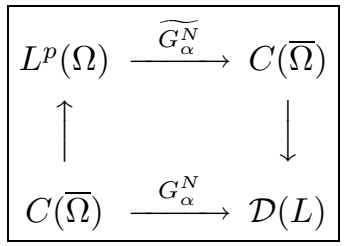

Fig. 3.1 The extension $\widetilde{G_{\alpha}^{N}}$ of the Green operator $G_{\alpha}^{N}$ to $L^{p}(\Omega)$

To prove assertion $(3.7)$ for all $v \in C^{2}(\bar{\Omega})$ such that

$$
\frac{\partial v}{\partial \mathbf{n}}=0 \quad \text { on } \partial \Omega
$$


we introduce an extension $\widetilde{G_{\alpha}^{N}}$ of the Green operator $G_{\alpha}^{N}$ to the space $L^{p}(\Omega)$ for $N<p<\infty$ (see Figure 3.1). By Theorem 3.1, we find that the Neumann problem

$$
\begin{cases}(\alpha+\mathcal{L}) u=f & \text { almost everywhere in } \Omega, \\ \frac{\partial u}{\partial \mathbf{n}}=0 & \text { on } \partial \Omega\end{cases}
$$

has a unique solution $u \in W^{2, p}(\Omega) \cap C^{1}(\bar{\Omega})$ for any $f \in L^{p}(\Omega)$. If we let

$$
u:=\widetilde{G_{\alpha}^{N}} f
$$

then it is easy to verify that the operator $\widetilde{G_{\alpha}^{N}}$ is an extension of $G_{\alpha}^{N}$ to $L^{p}(\Omega)$.

Moreover, just as in Steps 2 and 3, we can prove the following two assertions:

(A) The operator $\widetilde{G_{\alpha}^{N}}: L^{p}(\Omega) \rightarrow C(\bar{\Omega})$ is non-negative.

(B) The operator $\widetilde{G_{\alpha}^{N}}: L^{\infty}(\Omega) \rightarrow C(\bar{\Omega})$ is bounded with norm $1 / \alpha$ :

$$
\left\|\widetilde{G_{\alpha}^{N}}\right\| \leq \frac{1}{\alpha}
$$

First, since $a^{i j}, b^{i} \in L^{\infty}(\Omega)$ and $v \in C^{2}(\bar{\Omega})$, it follows that

$$
\mathcal{L} v=-\sum_{i, j=1}^{N} a^{i j}(x) \frac{\partial^{2} v}{\partial x_{i} \partial x_{j}}+\sum_{i=1}^{N} b^{i}(x) \frac{\partial v}{\partial x_{i}} \in L^{\infty}(\Omega) .
$$

Hence, if we let

$$
w:=\alpha G_{\alpha}^{N} v+\widetilde{G_{\alpha}^{N}}(\mathcal{L} v)
$$

then we have the assertions

$$
\begin{cases}w \in W^{2, p}(\Omega) \cap C^{1}(\bar{\Omega}) & \text { for } N<p<\infty, \\ (\mathcal{L}+\alpha) w=(\mathcal{L}+\alpha) v & \text { almost everywhere in } \Omega, \\ \frac{\partial w}{\partial \mathbf{n}}=0 & \text { on } \partial \Omega,\end{cases}
$$

and so

$$
\begin{cases}w-v \in W^{2, p}(\Omega) \cap C(\bar{\Omega}) & \text { for } N<p<\infty, \\ (\mathcal{L}+\alpha)(w-v)=0 & \text { almost everywhere in } \Omega, \\ \frac{\partial(w-v)}{\partial \mathbf{n}}=0 & \text { on } \partial \Omega .\end{cases}
$$

By applying Theorem 3.1 to the function $w(x)-v(x)$, we obtain that

$$
w-v=0 \quad \text { in } \Omega,
$$

that is,

$$
v=w=\alpha G_{\alpha}^{N} v+\widetilde{G_{\alpha}^{N}}(\mathcal{L} v) .
$$

Therefore, the desired assertion (3.7) follows from an application of assertion (B), since we have the inequality

$$
\left\|v-\alpha G_{\alpha}^{N} v\right\|_{C(\bar{\Omega})}=\left\|\widetilde{G_{\alpha}^{N}}(\mathcal{L} v)\right\|_{C(\bar{\Omega})} \leq \frac{1}{\alpha}\|\mathcal{L} v\|_{L^{\infty}(\Omega)} \quad \text { for all } \alpha>0 .
$$

Now the proof of Theorem 1.1 is complete. 


\subsection{Proof of Proposition 1.1}

The proof is divided into two steps (see [46, p. 461]).

Step 1: First, since $\mathcal{N}(L)=\operatorname{span}[1]$, we have the formula

$$
1=(L+I)^{-1} 1 \text {. }
$$

On the other hand, by Theorem 1.1 and assertion (3.6) with $\alpha:=1$ it follows that the resolvent

$$
R:=(L+I)^{-1}: Y \longrightarrow Y
$$

is compact and strongly positive. Hence, by applying the Kreĭn-Rutman theorem (Theorem 2.1) we obtain the following three assertions:

(1) $1=\operatorname{spr}(R)$.

(2) $\operatorname{dim} \mathcal{N}(L)=1 ;$ so $\operatorname{dim} \mathcal{N}(I-R)=1$.

(3) 1 is a simple eigenvalue of the Banach space adjoint operator

$$
R^{*}: Y^{*} \longrightarrow Y^{*}
$$

having a positive eigenfunction $\psi \in Y^{*}: R^{*} \psi=\psi$.

Indeed, it suffices to note that

$$
\begin{aligned}
& \psi=R^{*} \psi=\left((L+I)^{-1}\right)^{*} \psi=\left(L^{*}+I\right)^{-1} \psi \\
& \Longleftrightarrow\left(L^{*}+I\right) \psi=\psi \\
& \Longleftrightarrow L^{*} \psi=0 .
\end{aligned}
$$

Therefore, we have proved that

$$
\left\{\begin{array}{l}
\operatorname{dim} \mathcal{N}\left(L^{*}\right)=1, \\
\mathcal{N}\left(L^{*}\right)=\operatorname{span}[\psi]
\end{array}\right.
$$

Step 2: Secondly, we prove that $\psi \in Y^{*}$ can be identified with a positive function in $L^{q}(\Omega)$ for $q=p /(p-1)$ with $N<p<\infty$.

To do this, we introduce a densely defined, closed linear operator

$$
L^{\prime}: L^{p}(\Omega) \longrightarrow L^{p}(\Omega) \quad \text { for } N<p<\infty
$$

as follows:

(a) The domain $\mathcal{D}\left(L^{\prime}\right)$ of definition is the set

$$
\mathcal{D}\left(L^{\prime}\right)=\left\{u \in W^{2, p}(\Omega): \frac{\partial u}{\partial \mathbf{n}}=0 \text { on } \partial \Omega\right\}
$$

(b) $L^{\prime} u=\mathcal{L} u$ for every $u \in \mathcal{D}\left(L^{\prime}\right)$. 


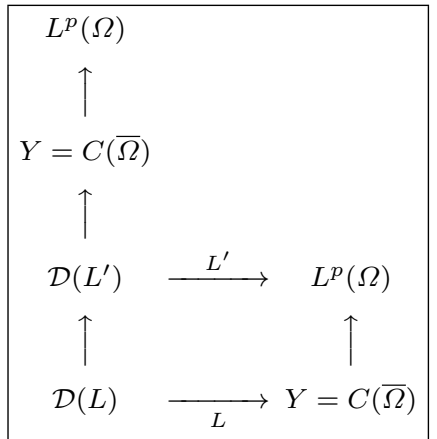

Fig. 3.2 The extension $L^{\prime}$ of $L$ to $L^{p}(\Omega)$

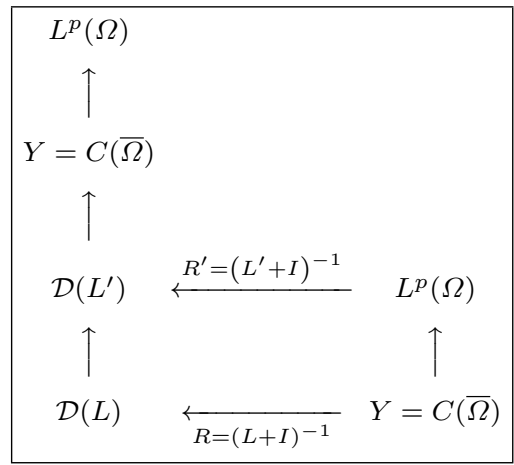

Fig. 3.3 The extension $R^{\prime}$ of $R$ to $L^{p}(\Omega)$

Then it is easy to see that the operator $L^{\prime}$ is an extension of $L$ and that the resolvent $R^{\prime}=\left(L^{\prime}+I\right)^{-1}$ is an extension of $R=(L+I)^{-1}$, respectively (see Figures 3.2 and 3.3).

Moreover, it follows from an application of the strong maximum principle (Theorem 3.3) that

$$
R^{\prime}:=\left(L^{\prime}+I\right)^{-1}: L^{p}(\Omega) \longrightarrow L^{p}(\Omega)
$$

is compact and strongly positive. Hence, by applying the KreĬn-Rutman theorem (Theorem 2.1) we obtain the following two assertions:

(4) $1=\operatorname{spr}\left(R^{\prime}\right)$.

(5) 1 is a simple eigenvalue of the Banach space adjoint operator

$$
\left(R^{\prime}\right)^{*}=\left(\left(L^{\prime}\right)^{*}+I\right)^{-1}: L^{q}(\Omega) \longrightarrow L^{q}(\Omega) \text { for } q=\frac{p}{p-1},
$$

having a positive eigenfunction $\psi^{\prime} \in L^{q}(\Omega)$ :

$$
\left(R^{\prime}\right)^{*} \psi^{\prime}=\psi^{\prime} \text {. }
$$

Then we have the assertion

$$
\psi^{\prime}=\left(R^{\prime}\right)^{*} \psi^{\prime}=\left(\left(L^{\prime}+I\right)^{-1}\right)^{*} \psi^{\prime}=\left(\left(L^{\prime}\right)^{*}+I\right)^{-1} \psi^{\prime}
$$




$$
\begin{aligned}
& \Longleftrightarrow\left(\left(L^{\prime}\right)^{*}+I\right) \psi^{\prime}=\psi^{\prime} \\
& \Longleftrightarrow \\
& \left(L^{\prime}\right)^{*} \psi^{\prime}=0 .
\end{aligned}
$$

On the other hand, since $R \subset R^{\prime}$, it follows that

$$
\left(R^{\prime}\right)^{*} \subset R^{*} \text {. }
$$

Indeed, it suffices to note two Figures 3.4 and 3.5.

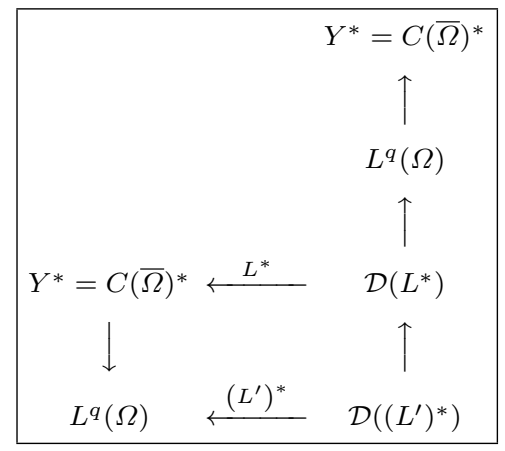

Fig. 3.4 The operators $L^{*}$ and $\left(L^{\prime}\right)^{*}$ with $q=p /(p-1)$

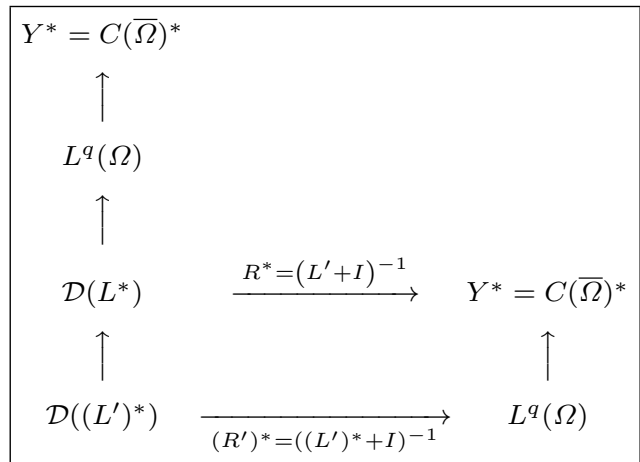

Fig. 3.5 The operators $R^{*}$ and $\left(R^{\prime}\right)^{*}$ with $q=p /(p-1)$

Hence we have the formula

$$
R^{*} \psi^{\prime}=\left(R^{\prime}\right)^{*} \psi^{\prime}=\psi^{\prime} .
$$

By assertion (3.10), this proves that

$$
\psi^{\prime} \in \mathcal{N}\left(L^{*}\right) .
$$


Therefore, by combining assertions (3.11) and (3.12) we can find a constant $c>0$ such that

$$
\psi^{\prime}=c \psi,
$$

since $\psi$ and $\psi^{\prime}$ are both positive. This proves that

$$
\psi=\frac{1}{c} \psi^{\prime} \in L^{q}(\Omega) .
$$

Now the proof of Proposition 1.1 is complete.

\section{Proof of parts (i) and (ii) of Theorem 1.2}

The proof of parts (i) and (ii) of Theorem 1.2 is divided into two subsections, due to its length. This section is the heart of the subject.

4.1 Spectrum of the Neumann eigenvalue problem

We look at the equation

$$
\widehat{L} v=\widehat{\lambda} \widehat{M} v \quad \text { in } \widehat{Y} \text { for } \widehat{\lambda} \in \mathbf{C},
$$

which is obtained by the complexification of the operator equation (1.5). Here and in the following the objects $\widehat{L}, \widehat{M}, \widehat{X}$ and $\widehat{Y}$ are defined as complexifications of $L$, $M, X:=\mathcal{D}(L)$ and $Y:=C(\bar{\Omega})$, respectively.

The purpose of this subsection is to study the spectrum $\sigma(\widehat{L}, \widehat{M})$ of $\widehat{L}$ with respect to $\widehat{M}$ under condition (M):

(M) The weight function $m(x) \in C(\bar{\Omega})$ changes sign in $\Omega$, that is, it takes both positive and negative values in $\Omega$.

We begin with the following:

Lemma 4.1 Under condition $(M)$, for any $\widehat{\lambda}_{0} \in \mathbf{C}$ the bounded operator

$$
\widehat{L}-\widehat{\lambda}_{0} \widehat{M}: \widehat{X} \longrightarrow \widehat{Y}
$$

is a Fredholm operator with index zero.

Proof We can write the operator $\widehat{L}-\widehat{\lambda}_{0} \widehat{M}$ in the form

$$
\widehat{L}-\widehat{\lambda}_{0} \widehat{M}=(\widehat{L}+\widehat{I})-\left(\widehat{\lambda}_{0} \widehat{M}+\widehat{I}\right) .
$$

However, it follows from an application of assertion (1.4) that the operator

$$
\widehat{\lambda}_{0} \widehat{M}+\widehat{I}: \widehat{X} \longrightarrow \widehat{Y}
$$

is compact, since $m \in C(\bar{\Omega})$.

On the other hand, by Theorem 1.1 with $\alpha:=1$ we obtain that the operator

$$
\widehat{L}+\widehat{I}: \widehat{X} \longrightarrow \widehat{Y}
$$

is an isomorphism. Hence we have, by the perturbation theory for Fredholm operators (see [26, Theorem 2.6], [43, Theorem 5.10]),

$$
\operatorname{ind}\left(\widehat{L}-\widehat{\lambda}_{0} \widehat{M}\right)=\operatorname{ind}(\widehat{L}+\widehat{I})=0 \text {. }
$$

The proof of Lemma 4.1 is complete. 
Now we consider the case where

$$
\int_{\Omega} m(x) \psi(x) d x<0 .
$$

Then we have the following lemma:

Lemma 4.2 Under conditions (M) and (4.1), the eigenvalue 0 is not an accumulation point of the spectrum $\sigma(\widehat{L}, \widehat{M})$. More precisely, every neighborhood of zero in $\mathbf{C}$ contains points of the resolvent set $\rho(\widehat{L}, \widehat{M})$ of $\widehat{L}$ with respect to $\widehat{M}$.

Proof The proof is based on a reduction to absurdity. Assume, to the contrary, that there exists a sequence $\left\{\widehat{\lambda}_{j}\right\} \subset \sigma(\widehat{L}, \widehat{M})$ such that

$$
\widehat{\lambda}_{j} \longrightarrow 0 \quad \text { as } j \rightarrow \infty \text {. }
$$

Then we can find a sequence $\left\{u_{j}\right\}$ in $\widehat{X}$ such that

$$
\left\{\begin{array}{l}
\widehat{L} u_{j}=\widehat{\lambda}_{j} \widehat{M} u_{j}, \\
\left\|u_{j}\right\|_{\widehat{Y}}=1 .
\end{array}\right.
$$

Indeed, by using Lemma 4.1 and the closed graph theorem (see [43, Theorem 3.10], [62, Chapter II, Section 6, Theorem 1]) we obtain that if the operator $\widehat{L}-\widehat{\lambda}_{j} \widehat{M}$ is injective, then $\widehat{\lambda}_{j} \in \rho(\widehat{L}, \widehat{M})$. Since we have the assertion

$$
\left\|\widehat{L} u_{j}\right\|_{\widehat{Y}}=\left\|\widehat{\lambda}_{j} \widehat{M} u_{j}\right\|_{\widehat{Y}} \leq\left|\widehat{\lambda}_{j}\right|\|\widehat{M}\|_{\widehat{Y}} \longrightarrow 0 \quad \text { as } j \rightarrow \infty,
$$

it follows that, for some constant $C_{1}>0$ independent of $j$,

$$
\left\|(\widehat{L}+\widehat{I}) u_{j}\right\|_{\widehat{Y}} \leq\left\|\widehat{L} u_{j}\right\|_{\widehat{Y}}+\left\|u_{j}\right\|_{\widehat{Y}} \leq C_{1}+1 .
$$

Moreover, since the operator

$$
\widehat{L}+\widehat{I}: \widehat{X} \longrightarrow \widehat{Y}
$$

is an isomorphism, we have, by inequality (4.2),

$$
\left\|u_{j}\right\|_{\widehat{X}} \leq C_{2},
$$

where $C_{2}>0$ is a constant independent of $j$.

Since the injection

$$
\widehat{I}: \widehat{X} \longrightarrow \widehat{Y}
$$

is compact, we can choose a subsequence $\left\{u_{j^{\prime}}\right\}$ of $\left\{u_{j}\right\}$ that converges to some function $u$ in $\widehat{Y}$. It should be noticed that

$$
\|u\|_{\widehat{Y}}=\lim _{j^{\prime} \rightarrow \infty}\left\|u_{j^{\prime}}\right\|_{\widehat{Y}}=1 .
$$

Summing up, we obtain that, as $j^{\prime} \rightarrow \infty$,

$$
\begin{array}{ll}
u_{j^{\prime}} \longrightarrow u & \text { in } \widehat{Y}, \\
\widehat{L} u_{j^{\prime}} \rightarrow 0 & \text { in } \widehat{Y} .
\end{array}
$$


By the closedness of $\widehat{L}$, this implies that

$$
\left\{\begin{array}{l}
u \in \mathcal{D}(\widehat{L})=\widehat{X}, \\
\widehat{L} u=0,
\end{array}\right.
$$

so that

$$
u \in \mathcal{N}(\widehat{L})=\operatorname{span}[1] .
$$

Therefore, by combining assertions (4.3) and (4.4) we conclude that

$$
u(x)= \pm 1 \quad \text { in } \Omega .
$$

(A) The case $u(x)=1$ in $\Omega$ : In this case, we have the assertion

$$
\left\langle\psi, \widehat{M} u_{j^{\prime}}\right\rangle \longrightarrow\langle\psi, M 1\rangle=\int_{\Omega} m(x) \psi(x) d x<0,
$$

and so

$$
\left\langle\psi, \widehat{M} u_{j^{\prime}}\right\rangle \neq 0 \quad \text { for } j^{\prime} \text { sufficiently large. }
$$

However, it follows that

$$
0=\left\langle\widehat{L}^{*} \psi, u_{j^{\prime}}\right\rangle=\left\langle\psi, \widehat{L} u_{j^{\prime}}\right\rangle=\widehat{\lambda}_{j^{\prime}}\left\langle\psi, \widehat{M} u_{j^{\prime}}\right\rangle \quad \text { for } \widehat{\lambda}_{j^{\prime}} \neq 0,
$$

so that

$$
\left\langle\psi, \widehat{M} u_{j^{\prime}}\right\rangle=0 \quad \text { for all } j^{\prime}
$$

This contradicts assertion (4.5).

(B) The case $u(x)=-1$ in $\Omega$ : The proof is essentially the same as that of case (A), since we have the formula

$$
\left\langle\psi, \widehat{M} u_{j^{\prime}}\right\rangle \longrightarrow-\langle\psi, M 1\rangle=-\int_{\Omega} m(x) \psi(x) d x>0 \quad \text { as } j^{\prime} \rightarrow \infty .
$$

The proof of Lemma 4.2 is complete.

Now we can prove the main result of this subsection:

Theorem 4.1 Under conditions $(M)$ and (4.1), the spectrum $\sigma(\widehat{L}, \widehat{M})$ is a countable set with no finite accumulation point.

Proof By Lemma 4.2, we can take a point $\widehat{\lambda}_{0}$ of $\rho(\widehat{L}, \widehat{M})$. If we let

$$
\widehat{T}=\left(\widehat{L}-\widehat{\lambda}_{0} \widehat{M}\right)^{-1} \widehat{M}: \widehat{Y} \longrightarrow \widehat{X},
$$

then we obtain that the operator

$$
\widehat{T}: \widehat{Y} \longrightarrow \widehat{Y}
$$

is compact, since the injection $\widehat{X} \rightarrow \widehat{Y}$ is compact. Moreover, it is easy to see that we have, for any $\widehat{\lambda} \neq \widehat{\lambda}_{0}$,

$$
\begin{aligned}
& (\widehat{L}-\widehat{\lambda} \widehat{M}) u=0 \quad \text { for } u \in \widehat{X}, \\
& \Longleftrightarrow \\
& \left(\widehat{I}-\left(\widehat{\lambda}-\widehat{\lambda}_{0} \widehat{T}\right) u=0 \quad \text { for } u \in \widehat{Y},\right.
\end{aligned}
$$




$$
\begin{aligned}
& \Longleftrightarrow \\
& \widehat{T} u=\left(\frac{1}{\widehat{\lambda}-\widehat{\lambda}_{0}}\right) u \quad \text { for } u \in \widehat{Y} .
\end{aligned}
$$

This proves that

$$
\widehat{\lambda} \in \sigma(\widehat{L}, \widehat{M}) \Longleftrightarrow \frac{1}{\widehat{\lambda}-\widehat{\lambda}_{0}} \in \sigma(\widehat{T}) \quad \text { for } \widehat{\lambda}_{0} \in \rho(\widehat{L}, \widehat{M}) .
$$

However, since $\widehat{T}$ is compact, it follows from an application of the Riesz-Schauder theory (see [62, Chapter X, Section 5, Theorem 3]) that the spectrum $\sigma(\widehat{T})$ is a countable set accumulating only at the zero. Therefore, we obtain from assertion (4.6) that the spectrum $\sigma(\widehat{L}, \widehat{M})$ is a countable set with no finite accumulation point.

The proof of Theorem 4.1 is complete.

\subsection{Proof of part (i) of Theorem 1.2}

In this subsection we prove part (i) of Theorem 1.2. The proof may be carried out just as in Senn-Hess [46, Theorem 2], by using Theorem 1.1 and the Kreĭn-Rutman theorem (Theorem 2.1).

By rescaling, we may assume that

$$
|m(x)|<1 \quad \text { on } \bar{\Omega} .
$$

The proof is divided into five steps.

Step 1: First, we prove the following fundamental lemma (cf. [28, Lemma 2]):

Lemma 4.3 If the function $m(x) \in C(\bar{\Omega})$ satisfies condition $(M)$, then there exist a constant $\alpha_{0}>0$ and a function $w_{0} \in P_{Y} \backslash\{0\}$ which satisfy the condition

$$
\alpha_{0} K_{\alpha_{0}} w_{0}-w_{0} \in P_{Y}
$$

where

$$
K_{\alpha_{0}}:=\left(\alpha_{0} I+L\right)^{-1}(M+I) .
$$

The proof of Lemma 4.3 is essentially the same as that of [50, Lemma 4.1] if we make use of Theorem 1.1 and the following Bakel'man and Aleksandrov weak maximum principle (cf. [10, Théorème 2], [25, Theorem 9.1], [50, Theorem 3.4]):

Theorem 4.2 (the weak maximum principle) Assume that

$$
\left\{\begin{array}{l}
u \in C(\bar{\Omega}) \cap W_{\mathrm{loc}}^{2, N}(\Omega), \\
\mathcal{L} u(x) \leq 0 \quad \text { for almost all } x \in \Omega .
\end{array}\right.
$$

Then it follows that

$$
\sup _{\Omega} u \leq \sup _{\partial \Omega} u^{+}
$$

where

$$
u^{+}(x)=\max \{u(x), 0\} \quad \text { for } x \in \bar{\Omega} .
$$


Step 2: The next lemma asserts that condition (4.8) implies the existence of a positive eigenvalue of the operator equation

$$
L u=\lambda M u \text {. }
$$

Lemma 4.4 Assume that the function $m(x) \in C(\bar{\Omega})$ satisfies conditions (M) and (4.1). If a constant $\alpha_{0}>0$ and a function $w_{0} \in P_{X} \backslash\{0\}$ satisfy the condition

$$
\alpha_{0} K_{\alpha_{0}} w_{0}-w_{0} \in P_{Y}
$$

then we can find a constant $\lambda \in\left(0, \alpha_{0}\right]$ and a function $u \in P_{X} \backslash\{0\}$ such that the operator equation (1.5) holds true.

Moreover, if we have the assertion

$$
\alpha_{0} K_{\alpha_{0}} w_{0}-w_{0} \in \operatorname{Int}\left(P_{Y}\right)
$$

then it follows that $0<\lambda<\alpha_{0}$.

The proof of Lemma 4.4 is essentially the same as that of Senn-Hess [46, Lemma 4] if we make use of Theorem 1.1 and the Kreĭn-Rutman theorem (Theorem 2.1).

Step 3: By Lemmas 4.3 and 4.4, we find that the set

$$
\Lambda(m)
$$

$=\{\lambda>0: \lambda$ is an eigenvalue of the equation (1.5) with a positive eigenfunction $\}$

is non-empty. If we let

$$
\lambda_{1}(m):=\inf \Lambda(m),
$$

then, by Theorem 4.1 we have the following lemma:

Lemma 4.5 Assume that conditions ( $M)$ and (4.1) are satisfied. Then the infimum in $\Lambda(m)$ is attained. Namely, $\lambda_{1}(m)$ is a positive eigenvalue of the operator equation (1.5) with a positive eigenfunction.

Proof Indeed, since we have the assertion

$$
\Lambda(m) \subset \sigma(\widehat{L}, \widehat{M})
$$

and since the spectrum $\sigma(\widehat{L}, \widehat{M})$ is a countable set with no finite accumulation point, it follows that

$$
\lambda_{1}(m) \in \Lambda(m) .
$$

The proof of Lemma 4.5 is complete.

Step 4: Now we look at the operator equation

$$
\widehat{L} v=\widehat{\lambda} \widehat{M} v \quad \text { in } \widehat{Y} \text { for } \widehat{\lambda} \in \mathbf{C},
$$

which is obtained by the complexification of the operator equation (1.5).

Substep 4-1: The next lemma plays a fundamental role in the proof of part (i) of Theorem 1.2 (cf. [46, Lemma 7]): 
Lemma 4.6 Assume that condition $(M)$ is satisfied. Let $\hat{\lambda}$ be an eigenvalue of the operator equation (1.7) with $\operatorname{Re} \widehat{\lambda}>0$, and let $v(x)$ be its associated eigenfunction. Then we have the inequality

$$
|v| \leq(\operatorname{Re} \widehat{\lambda}) K_{\operatorname{Re} \widehat{\lambda}}|v|
$$

where

$$
K_{\operatorname{Re} \widehat{\lambda}}=((\operatorname{Re} \widehat{\lambda}) I+L)^{-1}(M+I)
$$

Proof The proof of Lemma 4.6 is divided into three steps.

(1) If $u(x)$ is a complex-valued function in $W^{2, p}(\Omega)$ with $N<p<\infty$ and if $\varepsilon>0$, then we define a function $u_{\varepsilon} \in W^{2, p}(\Omega)$ by the formula

$$
u_{\varepsilon}(x)=\sqrt{|u(x)|^{2}+\varepsilon^{2}} .
$$

First, we prove (see Kato [30, Lemma 3]) that

$$
\mathcal{L} u_{\varepsilon} \leq \operatorname{Re}\left[\frac{\bar{u}}{u_{\varepsilon}} \cdot \mathcal{L} u\right]
$$

It should be noticed that inequality (4.11) asserts the difference of the both sides is a non-negative distribution.

Since the matrix $\left(a^{i j}\right)$ is positive definite and

$$
u_{\varepsilon}^{2}=u \cdot \bar{u}+\varepsilon^{2} \quad \text { for } \varepsilon>0,
$$

it follows that

$$
\begin{aligned}
\sum_{i, j=1}^{N} a^{i j}(x) \frac{\partial u_{\varepsilon}}{\partial x_{i}} \frac{\partial u_{\varepsilon}}{\partial x_{j}} & \leq \frac{|u|^{2}}{u_{\varepsilon}^{2}}\left(\sum_{i, j=1}^{N} a^{i j}(x) \frac{\partial u}{\partial x_{i}} \frac{\partial u}{\partial x_{j}}\right) \\
& \leq \sum_{i, j=1}^{N} a^{i j}(x) \frac{\partial u}{\partial x_{i}} \frac{\partial u}{\partial x_{j}}
\end{aligned}
$$

Moreover, we have the formulas

$$
\begin{aligned}
L\left(u_{\varepsilon}^{2}\right) & =2 u_{\varepsilon} \cdot \mathcal{L} u_{\varepsilon}-2 \sum_{i, j=1}^{N} a^{i j}(x) \frac{\partial u_{\varepsilon}}{\partial x_{i}} \frac{\partial u_{\varepsilon}}{\partial x_{j}} \\
& =\mathcal{L} u \cdot \bar{u}+u \cdot \overline{\mathcal{L} u}-2 \sum_{i, j=1}^{N} a^{i j}(x) \frac{\partial u}{\partial x_{i}} \frac{\partial u}{\partial x_{j}} \\
& =2 \operatorname{Re}[\mathcal{L} u \cdot \bar{u}]-2 \sum_{i, j=1}^{N} a^{i j}(x) \frac{\partial u}{\partial x_{i}} \frac{\partial u}{\partial x_{j}} .
\end{aligned}
$$

By dividing the both sides by $2 u_{\varepsilon}>0$, we obtain that

$$
\operatorname{Re}\left[\frac{\mathcal{L} u \cdot \bar{u}}{u_{\varepsilon}}\right]-\frac{1}{u_{\varepsilon}}\left(\sum_{i, j=1}^{N} a^{i j}(x) \frac{\partial u}{\partial x_{i}} \frac{\partial u}{\partial x_{j}}\right)
$$




$$
=\mathcal{L} u_{\varepsilon}-\frac{1}{u_{\varepsilon}}\left(\sum_{i, j=1}^{N} a^{i j}(x) \frac{\partial u_{\varepsilon}}{\partial x_{i}} \frac{\partial u_{\varepsilon}}{\partial x_{j}}\right) .
$$

Therefore, the desire inequality (4.11) follows by combining formula (4.13) and inequality (4.12). Indeed, we have the inequality

$$
\begin{aligned}
\operatorname{Re}\left[\frac{\mathcal{L} u \cdot \bar{u}}{u_{\varepsilon}}\right]-\mathcal{L} u_{\varepsilon} & =\frac{1}{u_{\varepsilon}}\left(\sum_{i, j=1}^{N} a^{i j}(x) \frac{\partial u}{\partial x_{i}} \frac{\partial u}{\partial x_{j}}-\sum_{i, j=1}^{N} a^{i j}(x) \frac{\partial u_{\varepsilon}}{\partial x_{i}} \frac{\partial u_{\varepsilon}}{\partial x_{j}}\right) \\
& \geq 0 .
\end{aligned}
$$

(2) If $\hat{\lambda}$ is an eigenvalue of the operator equation (1.7) and if $v(x)$ is its associated eigenfunction, then it follows that $v \in W^{2, p}(\Omega)$ with $N<p<\infty$. Hence, by applying inequality (4.11) to the function $v(x)$ we obtain that

$$
\mathcal{L} v_{\varepsilon} \leq \operatorname{Re}\left[\frac{\bar{v}}{v_{\varepsilon}} \cdot \mathcal{L} v\right]=\operatorname{Re}\left[\frac{\bar{v}}{v_{\varepsilon}} \cdot \widehat{\lambda} m(x) v\right]=(\operatorname{Re} \widehat{\lambda}) m(x)\left(\frac{|v|^{2}}{v_{\varepsilon}}\right),
$$

so that

$$
(\mathcal{L}+\operatorname{Re} \widehat{\lambda}) v_{\varepsilon} \leq(\operatorname{Re} \widehat{\lambda})\left(m(x) \cdot \frac{|v|^{2}}{v_{\varepsilon}}+v_{\varepsilon}\right) .
$$

However, we have the assertions

$$
\frac{\partial v_{\varepsilon}}{\partial \mathbf{n}}=\frac{\partial}{\partial \mathbf{n}}\left(\sqrt{|v|^{2}+\varepsilon^{2}}\right)=0
$$

and

$$
m(x) \cdot \frac{|v|^{2}}{v_{\varepsilon}}+v_{\varepsilon} \in C(\bar{\Omega}) .
$$

(3) By assertion (3.6) with $\alpha:=\operatorname{Re} \widehat{\lambda}$ (see Figure 3.1), we find that the Green operator

$$
\widetilde{G_{\operatorname{Re} \widehat{\lambda}}^{N}}=((\operatorname{Re} \widehat{\lambda}) I+\mathcal{L})^{-1}: L^{p}(\Omega) \longrightarrow C(\bar{\Omega})
$$

is non-negative, for $N<p<\infty$. Thus, by applying the Green operator $\widetilde{G_{\operatorname{Re} \widehat{\lambda}}^{N}}$ to the both sides of inequality (4.14) we obtain that

$$
v_{\varepsilon} \leq(\operatorname{Re} \widehat{\lambda})((\operatorname{Re} \widehat{\lambda}) I+L)^{-1}\left(m(x) \cdot \frac{|v|^{2}}{v_{\varepsilon}}+v_{\varepsilon}\right) .
$$

Therefore, the desired inequality (4.10) follows by passing to the limit $\varepsilon \downarrow 0$ in inequality (4.15), since we have the assertions

$$
\begin{aligned}
& v_{\varepsilon} \longrightarrow|v| \quad \text { in } C(\bar{\Omega}), \\
& \frac{|v|^{2}}{v_{\varepsilon}} \longrightarrow|v| \quad \text { in } C(\bar{\Omega}) .
\end{aligned}
$$

The proof of Lemma 4.6 is complete. 
Substep 4-2: By combining Lemmas 4.4 and 4.6, we obtain that if $\hat{\lambda}$ is an eigenvalue of the operator equation (1.7) and if $\operatorname{Re} \widehat{\lambda}>0$, then we have the inequality

$$
\operatorname{Re} \widehat{\lambda} \geq \lambda_{1}(m)
$$

Indeed, if $v(x)$ is an eigenfunction of the operator equation (1.7), then we have, by Lemma 4.6,

$$
|v| \leq(\operatorname{Re} \widehat{\lambda}) K_{\operatorname{Re} \widehat{\lambda}}|v|
$$

Therefore, by applying Lemma 4.4 with

$$
\alpha_{0}:=\operatorname{Re} \widehat{\lambda}, \quad w_{0}:=|v|,
$$

we can find a constant $\lambda \in(0, \operatorname{Re} \widehat{\lambda}]$ and a function $u \in P_{X} \backslash\{0\}$ such that

$$
L u=\lambda M u \quad \text { in } Y .
$$

This implies that $\lambda \in \Lambda(m)$, so that

$$
\lambda_{1}(m) \leq \lambda \leq \operatorname{Re} \widehat{\lambda} .
$$

The proof of property (b) of part (i) of Theorem 1.2 is complete.

Step 5: The difficulty in the proof of the rest of part (i) of Theorem 1.2 lies in the fact that the operator $L$ is not invertible. In the case where

$$
\int_{\Omega} m(x) \psi(x) d x<0
$$

we can reduce the operator equation (1.5) to an equivalent one. Indeed, if $\lambda^{\prime}$ is a number such that $0<\lambda^{\prime}<\lambda_{1}(m)$, we let

$$
L_{\lambda^{\prime}}:=L-\lambda^{\prime} M \text {. }
$$

Then it is easy to see that

$$
L u=\lambda M u \quad \text { in } Y \Longleftrightarrow\left\{\begin{array}{l}
L_{\lambda^{\prime}} u=\mu M u \quad \text { in } Y, \\
\mu=\lambda-\lambda^{\prime} .
\end{array}\right.
$$

The proof of property (a) of part (i) of Theorem 1.2 can be accomplished in a series of lemmas, just as in Senn-Hess [46]. In fact, we can prove the following three Lemmas 4.7, 4.8 and 4.9 (see [46, Lemmas 8, 9 and 10]):

Lemma 4.7 Assume that conditions (M) and (4.1) are satisfied. If $0<\lambda^{\prime}<\lambda_{1}(m)$, then, for any $\varepsilon \geq 0$ there exists an inverse $\left(L_{\lambda^{\prime}}+\varepsilon I\right)^{-1}: Y \rightarrow Y$, and it is strongly positive and compact.

Proof The proof of Lemma 4.7 is divided into three steps.

(1) By rescaling, we may assume that

$$
|m(x)|<1 \quad \text { on } \bar{\Omega} .
$$

Since $\lambda^{\prime}>0$, we can define an operator

$$
H_{\varepsilon}:=\left(L+\left(\varepsilon+\lambda^{\prime}\right) I\right)^{-1}(M+I)=G_{\varepsilon+\lambda^{\prime}}^{N}(M+I): Y \longrightarrow Y .
$$




$$
H_{\varepsilon}: Y \stackrel{M+I}{\longrightarrow} Y \stackrel{G_{\varepsilon+\lambda{ }^{\prime}}^{N}}{\longrightarrow} \underset{\text { compactly }}{\hookrightarrow \hookrightarrow} Y .
$$

Fig. 4.1 The mapping property of the operator $H_{\varepsilon}$

Then it follows that $H_{\varepsilon}$ is compact and strongly positive and further that

$$
\operatorname{spr}\left(H_{\varepsilon}\right):=\lim _{n \rightarrow \infty}\left\|H_{\varepsilon}^{n}\right\|^{1 / n}>0 .
$$

The situation can be visualized as in Figure 4.1.

(2) We let

and show that

$$
\alpha(\varepsilon):=\frac{1}{\operatorname{spr}\left(H_{\varepsilon}\right)}
$$

$$
\lambda^{\prime}<\alpha(\varepsilon) \text { for all } \varepsilon \geq 0 .
$$

The proof is based on a reduction to absurdity. Assume, to the contrary, that

$$
\lambda^{\prime} \geq \alpha\left(\varepsilon_{0}\right) \text { for some } \varepsilon_{0} \geq 0 .
$$

Then it follows from an application of the Kreı̆n-Rutman theorem (Theorem 2.1) that there exists a function $w \in P_{Y} \backslash\{0\}$ such that

$$
H_{\varepsilon_{0}} w=\frac{1}{\alpha\left(\varepsilon_{0}\right)} w \text { in } Y
$$

that is,

$$
\left(L+\left(\varepsilon_{0}+\lambda^{\prime}\right) I\right)^{-1}(M+I) w=\frac{1}{\alpha\left(\varepsilon_{0}\right)} w \quad \text { in } Y .
$$

Hence we have, by inequality (4.18),

$$
\left(L+\alpha\left(\varepsilon_{0}\right) I\right) w \leq\left(L+\left(\varepsilon_{0}+\lambda^{\prime}\right) I\right) w=\alpha\left(\varepsilon_{0}\right)(M+I) w \quad \text { in } Y .
$$

This proves that

$$
w \leq \alpha\left(\varepsilon_{0}\right)\left(L+\alpha\left(\varepsilon_{0}\right) I\right)^{-1}(M+I) w=\alpha\left(\varepsilon_{0}\right) K_{\alpha\left(\varepsilon_{0}\right)} w \text { in } Y .
$$

Therefore, by applying Lemma 4.4 with

$$
\alpha_{0}:=\alpha\left(\varepsilon_{0}\right), \quad w_{0}:=w,
$$

we can find an element $\lambda$ of $\Lambda(m)$ such that

$$
\lambda \in\left(0, \alpha\left(\varepsilon_{0}\right)\right] .
$$

By inequality (4.18), this implies a contradiction that

$$
\lambda_{1}(m) \leq \lambda \leq \alpha\left(\varepsilon_{0}\right) \leq \lambda^{\prime}<\lambda_{1}(m) .
$$

(3) For any $\varepsilon \geq 0$, it is easy to see that the equation

$$
\left(L_{\lambda^{\prime}}+\varepsilon I\right) u=h \quad \text { in } Y
$$


is equivalent to the equation

$$
\left(I-\lambda^{\prime} H_{\varepsilon}\right) u=\left(L+\left(\varepsilon+\lambda^{\prime}\right) I\right)^{-1} h \quad \text { in } Y .
$$

Hence, if there exists an inverse $\left(I-\lambda^{\prime} H_{\varepsilon}\right)^{-1}$, we have the formula

$$
u=\left(L_{\lambda^{\prime}}+\varepsilon I\right)^{-1} h=\left(I-\lambda^{\prime} H_{\varepsilon}\right)^{-1}\left(L+\left(\varepsilon+\lambda^{\prime}\right) I\right)^{-1} h \quad \text { in } Y .
$$

However, by assertion (4.17) it follows that

$$
\operatorname{spr}\left(\lambda^{\prime} H_{\varepsilon}\right)=\lambda^{\prime} \operatorname{spr}\left(H_{\varepsilon}\right)=\frac{\lambda^{\prime}}{\alpha(\varepsilon)}<1 .
$$

Therefore, we obtain that the inverse

$$
\left(I-\lambda^{\prime} H_{\varepsilon}\right)^{-1}
$$

exists and can be represented as a C. Neumann series

$$
\left(I-\lambda^{\prime} H_{\varepsilon}\right)^{-1}=\sum_{n=0}^{\infty}\left(\lambda^{\prime} H_{\varepsilon}\right)^{n} .
$$

This implies that the operator

$$
\left(I-\lambda^{\prime} H_{\varepsilon}\right)^{-1}: Y \rightarrow Y
$$

is bounded and strongly positive, since the operator $H_{\varepsilon}: Y \rightarrow Y$ is compact and strongly positive.

Summing up, we have proved that, for any $\varepsilon \geq 0$, the inverse

$$
\left(L_{\lambda^{\prime}}+\varepsilon I\right)^{-1}=\left(I-\lambda^{\prime} H_{\varepsilon}\right)^{-1} G_{\varepsilon+\lambda^{\prime}}^{N}: Y \longrightarrow Y
$$

exists, and is strongly positive and compact.

The proof of Lemma 4.7 is complete.

Now we consider the operator equation

$$
\left\{\begin{array}{l}
L_{\lambda^{\prime}} u=\mu M u, \\
\mu=\lambda-\lambda^{\prime}, \\
0<\lambda^{\prime}<\lambda_{1}(m),
\end{array}\right.
$$

which is equivalent to the operator equation (1.5). Then, just as in [46, Lemma 9] we can prove the following lemma:

Lemma 4.8 Under conditions ( $M$ ) and (4.1), the operator equation (4.19) has precisely two eigenvalues

$$
\mu_{1}(m)=\lambda_{1}(m)-\lambda^{\prime}>0,
$$

and

$$
\mu_{-1}(m)=-\lambda^{\prime}<0
$$

having positive eigenfunctions in $\operatorname{Int}\left(P_{X}\right)$. Moreover, the eigenvalues $1 / \mu_{1}(m)$ and $1 / \mu_{-1}(m)$ are eigenvalues of the operator

$$
L_{\lambda^{\prime}}^{-1} M: Y \longrightarrow Y
$$

with algebraic multiplicity one. 
Proof The proof of Lemma 4.8 may be carried out just as in the proof of Hess-Kato [28, Corollary 1 and Lemma 8] if we make use the following two assertions:

(i) For any $\varepsilon \geq 0$, the inverse $\left(L_{\lambda^{\prime}}+\varepsilon I\right)^{-1}$ exists, and is a strongly positive and compact operator in $Y$ (guaranteed by Lemma 4.7).

(ii) If $\widehat{\mu} \in \mathbf{C}$ is an eigenvalue of the equation

$$
\widehat{L}_{\lambda^{\prime}} v=\widehat{\mu} \widehat{M} v \quad \text { in } \widehat{Y} \text { for } \widehat{\mu} \in \mathbf{C}
$$

obtained by the complexification of equation (4.19) in $Y$ and if $\operatorname{Re} \widehat{\mu}>0$, then we have the inequality

$$
|v| \leq(\operatorname{Re} \widehat{\mu})\left(L_{\lambda^{\prime}}+(\operatorname{Re} \widehat{\mu}) I\right)^{-1}(M+I)|v| \quad \text { in } Y .
$$

The proof of inequality (4.21) is essentially the same as that of inequality (4.10) in Lemma 4.6, with $L$ replaced by $L_{\lambda^{\prime}}=L-\lambda^{\prime} M$.

The proof of Lemma 4.8 is complete.

Since we have the formula

$$
\lambda=\mu+\lambda^{\prime}
$$

we find from Lemma 4.8 that

$$
0=\mu_{-1}(m)+\lambda^{\prime}
$$

and

$$
\lambda_{1}(m)=\mu_{1}(m)+\lambda^{\prime}
$$

are the only eigenvalues of the operator equation (1.5) having positive eigenfunctions in Int $\left(P_{X}\right)$. Moreover, we can prove the following lemma (see [46, Lemma 10]):

Lemma 4.9 Assume that the function $m(x) \in C(\bar{\Omega})$ satisfies conditions $(M)$ and (4.1). Then the eigenvalues 0 and $\lambda_{1}(m)$ are $M$-simple eigenvalues of $L$ (see conditions (H.1) and (H.2) in Subsection 1.2):

(i) $\operatorname{dim} \mathcal{N}(L)=\operatorname{codim} \mathcal{R}(L)=1$.

(ii) If $\mathcal{N}(L)=\operatorname{span}\left[u_{1}\right]$, then $M u_{1} \notin \mathcal{R}(L)$.

(iii) $\operatorname{dim} \mathcal{N}\left(L-\lambda_{1}(m) M\right)=\operatorname{codim} \mathcal{R}\left(L-\lambda_{1}(m) M\right)=1$.

(iv) If $\mathcal{N}\left(L-\lambda_{1}(m) M\right)=\operatorname{span}\left[u_{2}\right]$, then $M u_{2} \notin \mathcal{R}\left(L-\lambda_{1}(m) M\right)$.

Proof The proof is divided into four steps.

Step 1 (Proof of assertion (i)): By assertion (ii) of Theorem 1.1, we find (see the proof of Lemma 4.1) that

$$
L: X \longrightarrow Y
$$

is a Fredholm operator with index zero, that is,

$$
\operatorname{dim} \mathcal{N}(L)=\operatorname{codim} \mathcal{R}(L) .
$$

If we let

$$
L_{\lambda^{\prime}}:=L-\lambda^{\prime} M \quad \text { for } 0<\lambda^{\prime}<\lambda_{1}(m),
$$


then we have, for $\mu_{-1}(m)=-\lambda^{\prime}$,

$$
L u=0 \Longleftrightarrow L_{\lambda^{\prime}} u=-\lambda^{\prime} M u \Longleftrightarrow\left(L_{\lambda^{\prime}}^{-1} M\right) u=\frac{1}{\mu_{-1}(m)} u .
$$

By Lemma 4.8, this proves that

$$
\operatorname{dim} \mathcal{N}(L)=1
$$

since $1 / \mu_{-1}(m)$ is a simple eigenvalue of $L_{\lambda^{\prime}}^{-1} M$.

Therefore, by combining assertions (4.22) and (4.24) we obtain that

$$
\operatorname{codim} \mathcal{R}(L)=1 \text {. }
$$

Step 2 (Proof of assertion (ii)): Now we assume that

$$
\mathcal{N}(L)=\operatorname{span}\left[u_{1}\right]
$$

Then we have, by formula (4.23) with $u:=u_{1}$,

$$
\left(L_{\lambda^{\prime}}^{-1} M\right) u_{1}=\frac{1}{\mu_{-1}(m)} u_{1} \text {. }
$$

The simplicity of the eigenvalue $1 / \mu_{-1}(m)$ implies that

$$
u_{1} \notin \mathcal{R}\left(L_{\lambda^{\prime}}^{-1} M-\frac{1}{\mu_{-1}(m)} I\right),
$$

so that

$$
M u_{1}=\frac{1}{\mu_{-1}(m)} L_{\lambda^{\prime}} u_{1} \notin \mathcal{R}\left(L_{\lambda^{\prime}}-\mu_{-1}(m) M\right)=\mathcal{R}(L) .
$$

Step 3 (Proof of assertion (iii)): Similarly, since the operator

$$
\lambda_{1}(m) M: X \longrightarrow Y
$$

is compact, we obtain that $L-\lambda_{1}(m) M$ is a Fredholm operator with index zero, that is,

$$
\operatorname{dim} \mathcal{N}\left(L-\lambda_{1}(m) M\right)=\operatorname{codim} \mathcal{R}\left(L-\lambda_{1}(m) M\right) .
$$

However, we have, by the Krĕn-Rutman theorem (Theorem 2.1),

$$
\operatorname{dim} \mathcal{N}\left(L-\lambda_{1}(m) M\right)=1
$$

Indeed, it suffices to note that

$$
\left(L-\lambda_{1}(m) M\right) u=0 \Longleftrightarrow K_{\lambda_{1}(m)} u=\frac{1}{\lambda_{1}(m)} u,
$$

and that

$$
\frac{1}{\lambda_{1}(m)}=\operatorname{spr}\left(K_{\lambda_{1}(m)}\right)
$$

is a simple eigenvalue of $K_{\lambda_{1}(m)}$, since $\lambda_{1}(m)$ is an eigenvalue of the operator equation (1.5) having a positive eigenfunction in $\operatorname{Int}\left(P_{X}\right)$.

Therefore, by combining assertions (4.25) and (4.26) we obtain that

$$
\operatorname{codim} \mathcal{R}\left(L-\lambda_{1}(m) M\right)=1 .
$$


Step 4 (Proof of assertion (iv)): Now we assume that

$$
\mathcal{N}\left(L-\lambda_{1}(m) M\right)=\operatorname{span}\left[u_{2}\right] .
$$

Then we have, for $\mu_{1}(m)=\lambda_{1}(m)-\lambda^{\prime}$,

$$
\begin{aligned}
\left(L-\lambda_{1}(m) M\right) u_{2}=0 & \Longleftrightarrow L_{\lambda^{\prime}} u_{2}=\mu_{1}(m) M u_{2} \\
& \Longleftrightarrow\left(L_{\lambda^{\prime}}^{-1} M\right) u_{2}=\frac{1}{\mu_{1}(m)} u_{2} .
\end{aligned}
$$

However, by Lemma 4.8 it follows that $1 / \mu_{1}(m)$ is an eigenvalue of $L_{\lambda^{\prime}}^{-1} M$ with algebraic multiplicity one. This implies that

$$
u_{2} \notin \mathcal{R}\left(L_{\lambda^{\prime}}^{-1} M-\frac{1}{\mu_{1}(m)} I\right)
$$

so that

$$
M u_{2}=\frac{1}{\mu_{1}(m)} L_{\lambda^{\prime}} u_{2} \notin \mathcal{R}\left(L_{\lambda^{\prime}}-\mu_{1}(m) M\right)=\mathcal{R}\left(L-\lambda_{1}(m) M\right) .
$$

The proof of Lemma 4.9 is complete.

The last Lemma 4.9 proves the rest of part (i) of Theorem 1.2.

4.3 Proof of part (ii) of Theorem 1.2

Now we consider the case where

$$
\int_{\Omega} m(x) \psi(x) d x>0 .
$$

However, the proof of part (ii) of Theorem 1.2 may be reduced to the case where

$$
\int_{\Omega} m(x) \psi(x) d x<0 .
$$

Indeed, it suffices to note that if $\int_{\Omega} m(x) \psi(x) d x>0$, then we have the assertions

$$
\left\{\begin{array}{l}
L u=\lambda M u=(-\lambda)(-M) u=\mu(-M) u \quad \text { in } Y, \\
\mu=-\lambda
\end{array}\right.
$$

under the condition that

$$
\int_{\Omega}(-m(x)) \psi(x) d x=-\int_{\Omega} m(x) \psi(x) d x<0 .
$$

Hence, it follows that

$$
\left\{\begin{array}{l}
L u=\mu_{1}(-m)(-M) u=-\mu_{1}(-m) M u \text { in } Y, \\
\mu_{1}(-m)>0 .
\end{array}\right.
$$

This proves that

$$
\lambda_{1}(m)=-\mu_{1}(-m)<0 .
$$

The proof of part (ii) of Theorem 1.2 is complete. 


\section{Proof of part (iii) of Theorem 1.2}

In this section we consider the case where

$$
\int_{\Omega} m(x) \psi(x) d x=0
$$

and prove part (iii) of Theorem 1.2 just as in Senn-Hess [46, Theorem 3].

Throughout this section, by rescaling, we may assume that

$$
|m(x)|<1 \quad \text { on } \bar{\Omega} \text {. }
$$

We remark that condition (5.1) implies that the function $m(x) \in C(\bar{\Omega})$ satisfies automatically condition (M), since $\psi(x)>0$ in $\Omega$.

First, it should be noticed that 0 is not an $M$-simple eigenvalue of $L$. Indeed, it suffices to note that condition (5.1) implies that

$$
M 1=m \in \mathcal{R}(L)
$$

since $\mathcal{N}\left(L^{*}\right)=\operatorname{span}[\psi]$.

The proof of part (iii) of Theorem 1.2 is divided into two subsections, due to its length.

5.1 Spectrum of the Neumann eigenvalue problem

The essential step in the proof is the following result, similar to Theorem 4.1:

Theorem 5.1 Under condition (5.1), the spectrum $\sigma(\widehat{L}, \widehat{M})$ is a countable set with no finite accumulation point. In particular, the eigenvalue 0 is an isolated point of $\sigma(\widehat{L}, \widehat{M})$.

Proof The proof is divided into four steps.

Step 1: First, we let

$$
\bar{t}:=\max _{x \in \bar{\Omega}} m^{+}(x)
$$

where

$$
m^{+}(x)=\max \{m(x), 0\},
$$

and consider the eigenvalue problem

$$
L u=\lambda(M-t I) u \quad \text { in } Y \text { for } 0<t<\bar{t} .
$$

Since the function $m(x)-t$ changes sign in $\Omega$ for $0<t<\bar{t}$ and

$$
\int_{\Omega}(m(x)-t) \psi(x) d x=-t \int_{\Omega} \psi(x) d x<0 \quad \text { for } 0<t<\bar{t},
$$

by applying part (i) of Theorem 1.2 with $m(x):=m(x)-t$ we obtain that the eigenvalue problem $(1.5)_{t}$ admits a unique positive eigenvalue

$$
\lambda_{t}:=\lambda_{1}(m-t) \quad \text { for } 0<t<\bar{t},
$$

with a positive eigenfunction $u_{t} \in \operatorname{Int}\left(P_{X}\right)$.

Moreover, it is strictly monotone increasing in $t \in I$. Indeed, it suffices to note the following comparison result for indefinite weight functions (see [46, Proposition 4]): 
Proposition 5.1 Let $m_{1}(x)$ and $m_{2}(x)$ be two weight functions in the ordered Banach space $Y=C(\bar{\Omega})$ such that

$$
m_{2}<m_{1} \text { in } Y \text {. }
$$

Assume that both $m_{1}(x)$ and $m_{2}(x)$ change sign in $\Omega$ and that

$$
\int_{\Omega} m_{1}(x) \psi(x) d x<0 .
$$

Then it follows that

$$
0<\lambda_{1}\left(m_{1}\right)<\lambda_{1}\left(m_{2}\right) .
$$

Proof By rescaling, we may assume that

$$
\left|m_{1}(x)\right|<1,\left|m_{2}(x)\right|<1 \text { on } \bar{\Omega} .
$$

Now let $u_{2}(x)$ be a positive eigenfunction corresponding to the eigenvalue $\lambda_{1}\left(m_{2}\right)$ :

$$
\begin{aligned}
& L u_{2}=\lambda_{1}\left(m_{2}\right) M_{2} u_{2}, \\
& u_{2}>0 .
\end{aligned}
$$

Then we have the formula

$$
\left(L+\lambda_{1}\left(m_{2}\right) I\right) u_{2}=\lambda_{1}\left(m_{2}\right)\left(M_{2}+I\right) u_{2},
$$

and so

$$
u_{2}=\lambda_{1}\left(m_{2}\right)\left(L+\lambda_{1}\left(m_{2}\right) I\right)^{-1}\left(\left(M_{2}+I\right) u_{2}\right),
$$

since $\lambda_{1}\left(m_{2}\right)>0$. However, by assertion (3.6) with $\alpha:=\lambda_{1}\left(m_{2}\right)$ we find that the operator

$$
\left(L+\lambda_{1}\left(m_{2}\right) I\right)^{-1}\left(M_{2}+I\right): Y \longrightarrow Y
$$

is strongly positive. Thus it follows from formula (5.2) that

$$
u_{2} \in \operatorname{Int}\left(P_{Y}\right) \text {, }
$$

so that

$$
\left(m_{2}+1\right) u_{2}<\left(m_{1}+1\right) u_{2} .
$$

Hence we have, by the strong positivity of $\left(L+\lambda_{1}\left(m_{2}\right) I\right)^{-1}$,

$$
\begin{aligned}
\lambda_{1}\left(m_{2}\right) K_{\lambda_{1}\left(m_{2}\right)} u_{2} & :=\lambda_{1}\left(m_{2}\right)\left(L+\lambda_{1}\left(m_{2}\right) I\right)^{-1}\left(\left(M_{1}+I\right) u_{2}\right) \\
& \gg \lambda_{1}\left(m_{2}\right)\left(L+\lambda_{1}\left(m_{2}\right) I\right)^{-1}\left(\left(M_{2}+I\right) u_{2}\right) \\
& =u_{2},
\end{aligned}
$$

and so

$$
\lambda_{1}\left(m_{2}\right) K_{\lambda_{1}\left(m_{2}\right)} u_{2}-u_{2} \in \operatorname{Int}\left(P_{Y}\right) .
$$

Therefore, by applying Lemma 4.4 with

$$
m(x):=m_{1}(x), \quad \alpha_{0}:=\lambda_{1}\left(m_{2}\right), \quad w_{0}:=u_{2},
$$

we can find an eigenvalue $\lambda \in\left(0, \lambda_{1}\left(m_{2}\right)\right)$ and an eigenfunction $u \in P_{X} \backslash\{0\}$ such that

$$
L u=\lambda M_{1} u .
$$

This proves that $\lambda \in \Lambda\left(m_{1}\right)$, so that

$$
\lambda_{1}\left(m_{1}\right) \leq \lambda<\lambda_{1}\left(m_{2}\right) .
$$

The proof of Proposition 5.1 is complete. 
We remark that the function $\lambda_{t}$ is continuous except at at most countably many points. Moreover, by using analytic perturbation theory due to Kato [31] just as in Hess-Kato [28, Lemma 4] (cf. [50, Lemma 5.2]), we can prove that the function $\lambda_{t}=\lambda_{1}(m-t)$ is a continuous function in $t \in I:=(0, \bar{t})$ (see Figure 5.1).

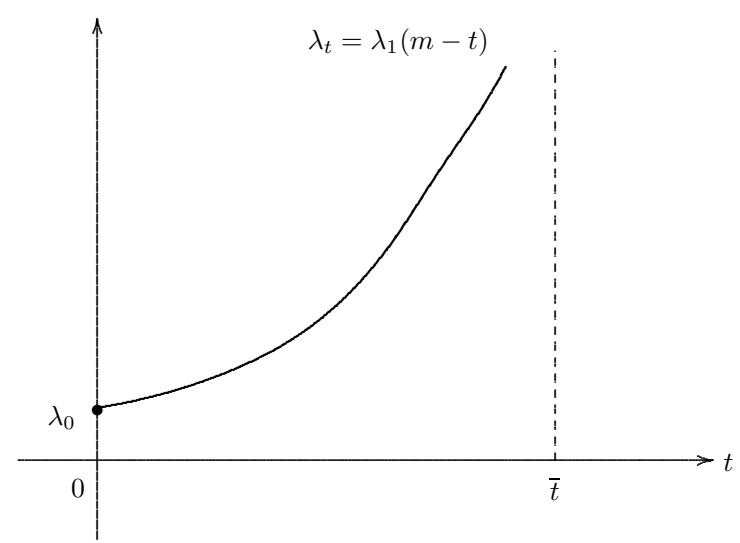

Fig. 5.1 The function $\lambda_{t}=\lambda_{1}(m-t)$ is strictly monotone increasing and continuous

Step 2: Secondly, we prove that

$$
\lim _{t \uparrow \bar{t}} \lambda_{t}=+\infty
$$

(1) In order to prove assertion (5.3), we need the following:

Claim 5.1 There is no eigenvalue $\lambda \neq 0$ of the operator equation $(1.5)_{t}$ having a positive eigenfunction for all $t \geq \bar{t}$.

Proof The proof is based on a reduction to absurdity. Assume, to the contrary, that there exists an eigenfunction $u \in P_{X} \backslash\{0\}$ such that

$$
L u=\lambda(M-t I) u \quad \text { in } Y \text { for some } \lambda \neq 0 .
$$

Then it follows that

$$
\left\{\begin{array}{l}
(L-M+t I) u=(1-\lambda)(t I-M) u \quad \text { in } Y \text { for } 0<t<\bar{t} \\
u \in P_{X} \backslash\{0\}
\end{array}\right.
$$

However, since $t-m(x) \geq 0$ in $\Omega$ for all $t \geq \bar{t}$, where

$$
\bar{t}=\max _{x \in \bar{\Omega}} m^{+}(x),
$$

we obtain that the inverse

$$
(L-M+t I)^{-1}: Y \longrightarrow Y
$$


exists and is strongly positive and compact (see the proof of formula (7.5)). By applying the Kreln-Rutman theorem (Theorem 2.1), we can find an eigenvalue $\gamma_{t}>0$ such that

$$
\left\{\begin{array}{l}
(L-M+t I) v_{t}=\gamma_{t}(t I-M) v_{t} \quad \text { in } Y \text { for } 0<t<\bar{t}, \\
v_{t} \in P_{X} \backslash\{0\} .
\end{array}\right.
$$

On the other hand, it is clear that

$$
\left\{\begin{array}{l}
(L-M+t I) 1=1(t I-M) 1 \quad \text { in } Y \text { for } 0<t<\bar{t}, \\
1 \in P_{X} \backslash\{0\} .
\end{array}\right.
$$

Therefore, by the uniqueness of principal eigenvalues it follows from formulas (5.4), (5.5) and (5.6) that

$$
1-\lambda=\gamma_{t}=1
$$

so that

$$
\lambda=0 .
$$

This contradiction proves Claim 5.1.

Remark 5.1 From the proof of Claim 5.1, we find that if the weight function $m(x) \in$ $C(\bar{\Omega})$ does not change sign in $\Omega$, then there is no eigenvalue $\lambda \neq 0$ of the operator equation

$$
L u=\lambda M u \quad \text { in } Y
$$

having a positive eigenfunction (see [46, p. 460]).

(2) Now the proof of the desired assertion (5.3) is based on a reduction to absurdity. Assume, to the contrary, that (see Figure 5.1)

$$
0<\bar{\lambda}:=\lim _{t \uparrow \bar{t}} \lambda_{t}<+\infty .
$$

By applying part (i) of Theorem 1.2 with $m(x):=m(x)-t$ for $0<t<\bar{t}$ we can find a function $u_{t} \in P_{X} \backslash\{0\}$ such that

$$
\begin{aligned}
& \left\{\begin{array}{l}
L u_{t}=\lambda_{t}(M-t I) u_{t} \quad \text { in } Y \text { for } 0<t<\bar{t}, \\
\left\|u_{t}\right\|_{Y}=1
\end{array}\right. \\
& \Longleftrightarrow \\
& \left\{\begin{array}{l}
\left(L+\lambda_{t} I\right) u_{t}=\lambda_{t}(M+I-t I) u_{t} \quad \text { in } Y \text { for } 0<t<\bar{t}, \\
\left\|u_{t}\right\|_{Y}=1 .
\end{array}\right.
\end{aligned}
$$

Moreover, since $\lambda_{t}>\lambda_{0}>0$, we have the formula

$$
\left\{\begin{array}{l}
u_{t}=\lambda_{t}\left(L+\lambda_{t} I\right)^{-1}(M+I-t I) u_{t} \quad \text { in } Y \text { for } 0<t<\bar{t} \\
\left\|u_{t}\right\|_{Y}=1
\end{array}\right.
$$

However, it is easy to verify the following three assertions:

(1) $\lambda_{t} \longrightarrow \bar{\lambda}$ as $t \uparrow \bar{t}$.

(2) $\left(L+\lambda_{t} I\right)^{-1} \longrightarrow(L+\bar{\lambda} I)^{-1}$ in $B(Y, Y)$ as $t \uparrow \bar{t}$. 
(3) $(L+\bar{\lambda} I)^{-1}(M+I): Y \longrightarrow Y$ is strongly positive and compact.

Here $B(Y, Y)$ is the Banach space of bounded (continuous) linear operators on $Y$.

Hence we can find an increasing sequence $t_{n} \uparrow \bar{t}$ and an element $\bar{v} \in Y$ such that

(a) $\lambda_{t_{n}} \longrightarrow \bar{\lambda}$ as $t_{n} \uparrow \bar{t}$.

(b) $\left(L+\lambda_{t_{n}} I\right)^{-1} \longrightarrow(L+\bar{\lambda} I)^{-1}$ in $B(Y, Y)$ as $t_{n} \uparrow \bar{t}$.

(c) $(L+\bar{\lambda} I)^{-1}(M+I) u_{t_{n}} \longrightarrow \bar{v}$ in $Y$ as $t_{n} \uparrow \bar{t}$.

If we let

$$
u_{\bar{t}}:=\bar{\lambda} \bar{v} \in Y,
$$

then we have, by formula (5.7) with $t:=t_{n}$ and assertions (a), (b) and (c),

$$
\begin{aligned}
u_{t_{n}}-u_{\bar{t}}= & \lambda_{t_{n}}\left(L+\lambda_{t_{n}} I\right)^{-1}\left(M+I-t_{n} I\right) u_{t_{n}}-\bar{\lambda} \bar{v} \\
= & \lambda_{t_{n}}\left(\left(L+\lambda_{t_{n}} I\right)^{-1}-(L+\bar{\lambda} I)^{-1}\right)\left(M+I-t_{n}\right) u_{t_{n}} \\
& +\lambda_{t_{n}}(L+\bar{\lambda} I)^{-1}\left(M+I-t_{n}\right) u_{t_{n}}-\bar{\lambda} \bar{v} \\
& \longrightarrow 0 \quad \text { in } Y \text { as } t_{n} \uparrow \bar{t}
\end{aligned}
$$

Therefore, by passing to the limit $t_{n} \uparrow \bar{t}$ in formula (5.7) we find that

$$
\begin{aligned}
& \left\{\begin{array}{l}
u_{\bar{t}}=\bar{\lambda}(L+\bar{\lambda} I)^{-1}(M+I-\bar{t}) u_{\bar{t}} \quad \text { in } Y, \\
u_{\bar{t}} \in P_{X} \backslash\{0\}, \\
\left\|u_{\bar{t}}\right\|_{Y}=1
\end{array}\right. \\
& \Longleftrightarrow \begin{array}{l}
L u_{\bar{t}}=\bar{\lambda}(M-\bar{t} I) u_{\bar{t}} \quad \text { in } Y, \\
u_{\bar{t}} \in P_{X} \backslash\{0\}, \\
\left\|u_{\bar{t}}\right\|_{Y}=1 .
\end{array}
\end{aligned}
$$

However, we have, by Claim 5.1 with $t:=\bar{t}$ and $\lambda:=\bar{\lambda}$,

$$
\bar{\lambda}=0 .
$$

This contradiction proves the desired assertion (5.3).

(3) Summing up, we have proved (see Figure 5.1) that the function $\lambda_{t}:=$ $\lambda_{1}(m-t)$ is strictly monotone increasing and continuous in $t \in I:=(0, \bar{t})$, and

$$
\begin{aligned}
& \lim _{t \uparrow \bar{t}} \lambda_{t}=+\infty, \\
& \lambda_{0}:=\lim _{t \downarrow 0} \lambda_{t} \geq 0 .
\end{aligned}
$$

Step 3: Moreover, we have the following lemma:

Lemma 5.1 Under condition (5.1), there exists a function $u_{0} \in P_{X} \backslash\{0\}$ such that

$$
L u_{0}=\lambda_{0} M u_{0} \quad \text { in } Y .
$$


Proof (1) If $\lambda_{0}=0$, we can take

$$
u_{0}=1 \text {. }
$$

(2) If $\lambda_{0}>0$, then we can find a function $u_{t} \in P_{X} \backslash\{0\}, 0<t<\bar{t}$, such that

$$
\left\{\begin{array}{l}
L u_{t}=\lambda_{t}(M-t I) u_{t} \quad \text { in } Y, \\
\left\|u_{t}\right\|_{Y}=1
\end{array}\right.
$$

Hence we have the formula

$$
\left(L+\lambda_{t} I\right) u_{t}=\lambda_{t}(M+I-t I) u_{t} \quad \text { in } Y \text { for } 0<t<\bar{t},
$$

and so

$$
\left\{\begin{array}{l}
u_{t}=\lambda_{t}\left(L+\lambda_{t} I\right)^{-1}(M+I-t I) u_{t} \quad \text { in } Y \text { for } 0<t<\bar{t}, \\
\left\|u_{t}\right\|_{Y}=1 .
\end{array}\right.
$$

since $\lambda_{t}>\lambda_{0}>0$ (see Figure 5.1).

However, it is easy to verify the following three assertions:

(1) $\lambda_{t} \longrightarrow \lambda_{0}$ as $t \downarrow 0$.

(2) $\left(L+\lambda_{t} I\right)^{-1} \longrightarrow\left(L+\lambda_{0} I\right)^{-1}$ in $B(Y, Y)$ as $t \downarrow 0$.

(3) $\left(L+\lambda_{0} I\right)^{-1}(M+I): Y \longrightarrow Y$ is strongly positive and compact.

Hence we can find an increasing sequence $t_{n} \downarrow 0$ and an element $v \in Y$ such that

(a) $\lambda_{t_{n}} \longrightarrow \lambda_{0}$ as $t_{n} \downarrow 0$.

(b) $\left(L+\lambda_{t_{n}} I\right)^{-1} \longrightarrow\left(L+\lambda_{0} I\right)^{-1}$ in $B(Y, Y)$ as $t_{n} \downarrow 0$.

(c) $\left(L+\lambda_{0} I\right)^{-1}(M+I) u_{t_{n}} \longrightarrow v$ in $Y$ as $t_{n} \downarrow 0$.

If we let

$$
u_{0}:=\lambda_{0} v \in Y,
$$

then we have, by formula (5.9) with $t:=t_{n}$ and assertions (a), (b) and (c),

$$
u_{t_{n}}-u_{0} \longrightarrow 0 \quad \text { in } Y \text { as } t_{n} \downarrow 0,
$$

just as in Step 2.

Therefore, by passing to the limit $t \downarrow 0$ in formula (5.9) we can find that

$$
\begin{aligned}
&\left\{\begin{array}{l}
u_{0}=\lambda_{0}\left(L+\lambda_{0} I\right)^{-1}(M+I) u_{0} \quad \text { in } Y, \\
u_{0} \in P_{X} \backslash\{0\}, \\
\left\|u_{0}\right\|_{Y}=1
\end{array}\right. \\
& \Longleftrightarrow \\
&\left\{\begin{array}{l}
L u_{0}=\lambda_{0} M u_{0} \text { in } Y, \\
u_{0} \in P_{X} \backslash\{0\}, \\
\left\|u_{0}\right\|_{Y}=1 .
\end{array}\right.
\end{aligned}
$$

This proves the desired equation (5.8).

The proof of Lemma 5.1 is complete.

Step 4: The next lemma proves Theorem 5.1: 
Lemma 5.2 Under condition (5.1), the spectrum $\sigma(\widehat{L}, \widehat{M})$ does not coincide with the whole $\mathbf{C}$.

Proof The proof of Lemma 5.2 is divided into two steps.

(1) First, we show that all real numbers $\lambda$ in a neighborhood of $\lambda_{0}$ admit positive eigenfunctions of the Neumann eigenvalue problem (1.5).

The proof is based on a reduction to absurdity. Assume, to the contrary, that there exist a sequence $\left\{\lambda_{j}\right\}$ in $\mathbf{R}$ and a sequence $\left\{u_{j}\right\}$ in $X$ such that

$$
\lambda_{j} \longrightarrow \lambda_{0} \quad \text { as } j \rightarrow \infty,
$$

and further that

$$
\left\{\begin{array}{l}
L u_{j}=\lambda_{j} M u_{j} \quad \text { in } Y, \\
\left\|u_{j}\right\|_{Y}=1 .
\end{array}\right.
$$

(A) The case where $\lambda_{0}=0$ : In this case, we may assume that

$$
u_{j} \longrightarrow 1 \text { in } Y \text {. }
$$

Indeed, since we have, by condition (4.7),

$$
\left\|L u_{j}\right\|_{Y}=\left\|\lambda_{j} M u_{j}\right\|_{Y} \leq\left|\lambda_{j}\right|\|M\|_{Y} \leq\left|\lambda_{j}\right| \longrightarrow 0 \quad \text { as } j \rightarrow \infty .
$$

it follows that, for some constant $C_{1}>0$ independent of $j$,

$$
\left\|(L+I) u_{j}\right\|_{Y} \leq\left\|L u_{j}\right\|_{Y}+\left\|u_{j}\right\|_{Y} \leq C_{1}+1 .
$$

However, since the operator

$$
L+I: X \longrightarrow Y
$$

is an isomorphism, we have, by inequality (5.11),

$$
\left\|u_{j}\right\|_{X} \leq C_{2},
$$

where $C_{2}>0$ is a constant independent of $j$. Moreover, since the injection $X \rightarrow Y$ is compact, we can choose a subsequence $\left\{u_{j^{\prime}}\right\}$ which converges to some function $u$ in $Y$. We remark that

$$
\|u\|_{Y}=\lim _{j^{\prime} \rightarrow \infty}\left\|u_{j^{\prime}}\right\|_{Y}=1
$$

Summing up, we obtain that

$$
\begin{aligned}
& u_{j^{\prime}} \rightarrow u \quad \text { in } Y, \\
& L u_{j^{\prime}} \rightarrow 0 \quad \text { in } Y .
\end{aligned}
$$

By the closedness of $L$, this implies that

$$
\left\{\begin{array}{l}
u \in \mathcal{D}(L)=X, \\
L u=0,
\end{array}\right.
$$

so that

$$
u \in \mathcal{N}(L)=\operatorname{span}[1]
$$


Therefore, by combining assertions (5.12) and (5.13) we conclude (if necessary by changing sign in $u_{j^{\prime}}$ ) that

$$
u(x)=1 \quad \text { in } \Omega .
$$

This proves the desired assertion (5.10).

By assertion (5.10), we may assume that, for $j^{\prime}$ sufficiently large,

$$
u_{j^{\prime}} \in \operatorname{Int}\left(P_{Y}\right) \text {. }
$$

(B) The case where $\lambda_{0}>0$ : In this case, there exists a function $u_{0} \in \operatorname{Int}\left(P_{Y}\right)$ such that

$$
u_{j} \longrightarrow u_{0} \quad \text { in } Y \text {. }
$$

Indeed, we have the formulas

$$
\left(L+\lambda_{j} I\right) u_{j}=\lambda_{j}(M+I) u_{j} \quad \text { in } Y,
$$

and so

$$
u_{j}=\lambda_{j}\left(L+\lambda_{j} I\right)^{-1}(M+I) u_{j}=\lambda_{j} K_{\lambda_{j}} u_{j} \quad \text { in } Y,
$$

since $\lambda_{j}>0$.

However, it is easy to verify the following two assertions:

(a) $K_{\lambda_{j}}=\left(L+\lambda_{j} I\right)^{-1}(M+I) \longrightarrow K_{\lambda_{0}}=\left(L+\lambda_{0} I\right)^{-1}(M+I)$ in $B(Y, Y)$ as $j \rightarrow \infty$

(b) $K_{\lambda_{0}}=G_{\lambda_{0}}^{N}(M+I): Y \rightarrow Y$ is compact.

Therefore, since $\left\|u_{j}\right\|_{Y}=1$, we can choose a subsequence $\left\{u_{j^{\prime}}\right\}$ that converges to some function $\widetilde{u}_{0}$ in $Y$ :

$$
u_{j^{\prime}} \longrightarrow \widetilde{u}_{0} \quad \text { in } Y \text { as } j^{\prime} \rightarrow \infty .
$$

Since $\lambda_{j} \rightarrow \lambda_{0}$ as $j \rightarrow \infty$, by passing to the limit $j^{\prime} \rightarrow \infty$ in formula (5.15) we obtain that

$$
\widetilde{u}_{0}=\lambda_{0} K_{\lambda_{0}} \widetilde{u}_{0}=\widetilde{u}_{0}\left(L+\lambda_{0} I\right)^{-1}(M+I) \widetilde{u}_{0} \quad \text { in } Y,
$$

so that

$$
\left\{\begin{array}{l}
L \widetilde{u}_{0}=\lambda_{0} M \widetilde{u}_{0} \quad \text { in } Y, \\
\left\|\widetilde{u}_{0}\right\|_{Y}=1 .
\end{array}\right.
$$

On the other hand, by Lemma 5.1 it follows that there exists a function $u_{0} \in$ $P_{X} \backslash\{0\}$ such that

$$
L u_{0}=\lambda_{0} M u_{0} \quad \text { in } Y,
$$

or equivalently,

$$
u_{0}=\lambda_{0}\left(L+\lambda_{0} I\right)^{-1}(M+I) u_{0}=\lambda_{0} K_{\lambda_{0}} u_{0} \quad \text { in } Y .
$$

However, we have, by the Krel̆n-Rutman theorem (Theorem 2.1),

$$
\operatorname{dim} \mathcal{N}\left(L-\lambda_{0} M\right)=1 .
$$

Indeed, it suffices to note that

$$
\left(L-\lambda_{0} M\right) u=0 \Longleftrightarrow K_{\lambda_{0}} u=\frac{1}{\lambda_{0}} u,
$$


and that $1 / \lambda_{0}=\operatorname{spr}\left(K_{\lambda_{0}}\right)$ is a simple eigenvalue of $K_{\lambda_{0}}$.

Therefore, by combining assertions (5.16), and (5.8) and (5.17) we conclude (if necessary by changing sign in $u_{j^{\prime}}$ ) that

$$
\widetilde{u}_{0}=u_{0} \in \operatorname{Int}\left(P_{Y}\right) .
$$

This proves the desired assertion (5.14).

By assertion (5.14), we may assume that, for $j^{\prime}$ sufficiently large,

$$
u_{j^{\prime}} \in \operatorname{Int}\left(P_{Y}\right)
$$

(2) By Step (1), if $\widetilde{\lambda}>\lambda_{0}$ is sufficiently close to $\lambda_{0}$, then there exists a function $\widetilde{u} \in \operatorname{Int}\left(P_{Y}\right)$ such that

$$
L \widetilde{u}=\widetilde{\lambda} M \widetilde{u} \quad \text { in } Y .
$$

It should be noticed (see Figure 5.2) that $\widetilde{\lambda}=\lambda_{1}(m-t)$ for some $t \in(0, \bar{t})$. Hence it follows that there exists a function $u_{t} \in \operatorname{Int}\left(P_{Y}\right)$ such that

$$
L u_{t}=\tilde{\lambda}(M-t I) u_{t} \quad \text { in } Y .
$$

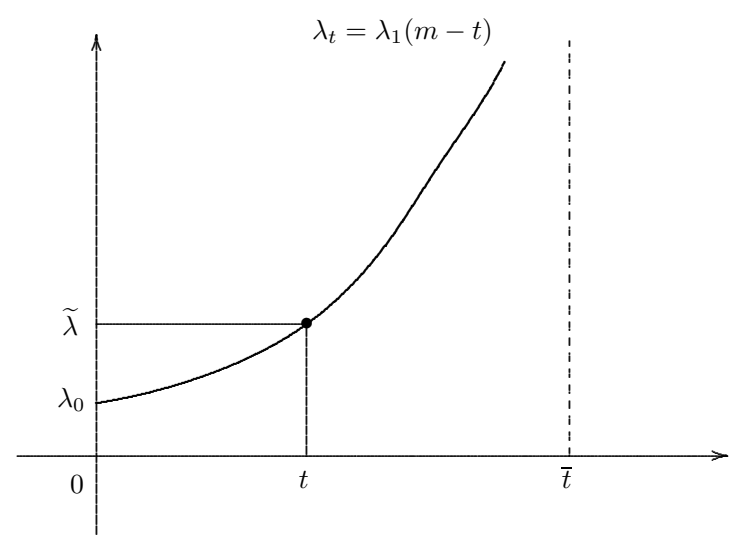

Fig. 5.2 $\tilde{\lambda}=\lambda_{1}(m-t)$ for some $t \in(0, \bar{t})$

Since $\widetilde{u}, u_{t} \in \operatorname{Int}\left(P_{Y}\right)$, we can find a constant $\sigma>0$ such that

$$
\widetilde{u}-\sigma u_{t} \in \partial P_{Y} .
$$

Then we have the inequality

$$
\begin{aligned}
(L+\widetilde{\lambda} I)\left(\widetilde{u}-\sigma u_{t}\right) & =\widetilde{\lambda} M \widetilde{u}-\sigma \widetilde{\lambda}(M-t I) u_{t}+\widetilde{\lambda} \widetilde{u}-\sigma \widetilde{\lambda} u_{t} \\
& =\widetilde{\lambda}(M+I) \widetilde{u}-\sigma \widetilde{\lambda}(M+I) u_{t}+\sigma \widetilde{\lambda} t u_{t} \\
& =\widetilde{\lambda}(M+I)\left(\widetilde{u}-\sigma \widetilde{\lambda} u_{t}\right)+\sigma \widetilde{\lambda} t u_{t} \\
& \geq \sigma \widetilde{\lambda} t u_{t} \quad \text { in } Y .
\end{aligned}
$$


However, by the strong positivity of $G_{\tilde{\lambda}}^{N}=(L+\widetilde{\lambda} I)^{-1}$ it follows that

$$
\widetilde{u}-\sigma u_{t} \in \operatorname{Int}\left(P_{Y}\right) \text {. }
$$

This contradicts condition (5.18).

The proof of Lemma 5.2 and hence that of Theorem 5.1 is complete.

\subsection{End of proof of part (iii) of Theorem 1.2}

The proof of part (iii) of Theorem 1.2 is based on a reduction to absurdity. Assume, to the contrary, that the operator equation (1.5) admits non-zero eigenvalues having positive eigenfunctions. By Theorem 5.1, we can find the smallest one $\lambda^{*}$ among positive eigenvalues. If $\lambda^{\prime}$ is a number such that $0<\lambda^{\prime}<\lambda^{*}$, we let

$$
L_{\lambda^{\prime}}:=L-\lambda^{\prime} M \text {. }
$$

Step 1: First, we have the following lemma (cf. [46, Lemma 13]), similar to Lemma 4.7:

Lemma 5.3 For any $\varepsilon \geq 0$, there exists an inverse

$$
\left(L_{\lambda^{\prime}}+\varepsilon I\right)^{-1}: Y \longrightarrow Y
$$

and it is strongly positive and compact for $0<\lambda^{\prime}<\lambda^{*}$.

Proof By using assertion (3.6), for each $\varepsilon \geq 0$ we can define a compact and strongly positive operator (see Figure 4.1)

$$
H_{\varepsilon}:=\left(L+\left(\varepsilon+\lambda^{\prime}\right) I\right)^{-1}(M+I)=G_{\varepsilon+\lambda^{\prime}}^{N}(M+I): Y \longrightarrow Y .
$$

Then we let

$$
\alpha(\varepsilon):=\frac{1}{\operatorname{spr}\left(H_{\varepsilon}\right)} .
$$

Since the Kreln-Rutman theorem (Theorem 2.1) guarantees uniqueness of the positive eigenvalues having positive eigenfunctions for compact strongly positive operators, we find that $\alpha(\varepsilon)$ is a continuous function of $\varepsilon$.

The proof of Lemma 5.3 is divided into two steps.

(1) First, we show that

$$
\lambda^{\prime}<\alpha(\varepsilon) \text { for all } \varepsilon \geq 0 .
$$

The proof is based on a reduction to absurdity. Assume, to the contrary, that

$$
\lambda^{\prime} \geq \alpha\left(\varepsilon_{0}\right) \text { for some } \varepsilon_{0}>0 .
$$

Then it follows from an application of the Krel̆n-Rutman theorem that there exists a function $w \in P_{Y} \backslash\{0\}$ such that

$$
H_{\varepsilon_{0}} w=\frac{1}{\alpha\left(\varepsilon_{0}\right)} w \quad \text { in } Y
$$

that is,

$$
\left(L+\left(\varepsilon_{0}+\lambda^{\prime}\right) I\right)^{-1}(M+I) w=\frac{1}{\alpha\left(\varepsilon_{0}\right)} w \quad \text { in } Y .
$$


Hence we have, by inequality (5.21),

$$
\left(L+\left(\varepsilon_{0}+\alpha\left(\varepsilon_{0}\right)\right) I\right) w \leq\left(L+\left(\varepsilon_{0}+\lambda^{\prime}\right) I\right) w=\alpha\left(\varepsilon_{0}\right)(M+I) w \quad \text { in } Y .
$$

This proves that

$$
w \leq \alpha\left(\varepsilon_{0}\right)\left(\left(L+\left(\varepsilon_{0}+\alpha\left(\varepsilon_{0}\right)\right) I\right)\right)^{-1}(M+I) w \quad \text { in } Y .
$$

Therefore, by applying Lemma 4.4 with

$$
L:=L+\varepsilon_{0} I, \quad \alpha_{0}:=\alpha\left(\varepsilon_{0}\right), \quad w_{0}:=w,
$$

we can find an eigenvalue $\lambda_{1, \varepsilon_{0}}(m) \in\left(0, \alpha\left(\varepsilon_{0}\right)\right]$ and an eigenfunction $u \in P_{X} \backslash\{0\}$ such that

$$
\left(L+\varepsilon_{0} I\right) u=\lambda_{1, \varepsilon_{0}}(m) M u \text { in } Y .
$$

However, by inequality (5.21) it follows that

$$
\alpha\left(\varepsilon_{0}\right) \leq \lambda^{\prime}<\lambda^{*}
$$

Hence we have the inequality

$$
\lambda_{1, \varepsilon_{0}}(m) \leq \alpha\left(\varepsilon_{0}\right)<\lambda^{*} .
$$

On the other hand, if $v \in P_{X} \backslash\{0\}$ is a positive eigenfunction of the eigenvalue problem

$$
L v=\lambda^{*} M v \text { in } Y,
$$

then it follows that

$$
\left(L+\varepsilon_{0} I\right) v=\lambda^{*}\left(M+\frac{\varepsilon_{0}}{\lambda^{*}} I\right) v \quad \text { in } Y .
$$

Therefore, by applying Proposition 5.1 with

$$
\begin{aligned}
& m_{1}(x):=m(x)+\frac{\varepsilon_{0}}{\lambda^{*}}, \\
& m_{2}(x):=m(x),
\end{aligned}
$$

we obtain from equations (5.22) and (5.24) that

$$
\lambda^{*}=\lambda_{1, \varepsilon_{0}}\left(m+\frac{\varepsilon_{0}}{\lambda^{*}}\right)<\lambda_{1, \varepsilon_{0}}(m) .
$$

This contradicts assertion (5.23).

Summing up, we have proved that

$$
\lambda^{\prime}<\alpha(\varepsilon) \text { for all } \varepsilon>0 .
$$

By passing to the limit $\varepsilon \downarrow 0$ in assertion (5.25), we obtain that

$$
\lambda^{\prime} \leq \alpha(0) .
$$

However, we have the assertion

$$
\lambda^{\prime}<\alpha(0) \text {. }
$$


Indeed, if $\lambda^{\prime}=\alpha(0)$, then it follows from formula (5.19) with $\varepsilon:=0$ that there exists an eigenfunction $w \in P_{X} \backslash\{0\}$ such that

$$
\left(L+\lambda^{\prime} I\right)^{-1}(M+I) w=\frac{1}{\alpha(0)} w \quad \text { in } Y,
$$

so that

$$
\left(L+\lambda^{\prime} I\right) w=\alpha(0)(M+I) w=\lambda^{\prime} M w+\lambda^{\prime} w \quad \text { in } Y .
$$

In particular, we have the assertions

$$
\left\{\begin{array}{l}
L w=\lambda^{\prime} M w \quad \text { in } Y, \\
w \in P_{X} \backslash\{0\} .
\end{array}\right.
$$

This contradicts the choice of $\lambda^{\prime}$, since $0<\lambda^{\prime}<\lambda^{*}$ and $\lambda^{*}$ is the smallest one among positive eigenvalues of the Neumann eigenvalue problem (1.5) having positive eigenfunctions.

Therefore, the desired assertion (5.20) follows by combining assertions (5.25) and (5.26).

(2) Secondly, for any $\varepsilon \geq 0$ it is easy to see that the equation

$$
\left(L_{\lambda^{\prime}}+\varepsilon I\right) u=h \quad \text { in } Y
$$

is equivalent to the equation

$$
\left(I-\lambda^{\prime} H_{\varepsilon}\right) u=\left(L+\left(\varepsilon+\lambda^{\prime}\right) I\right)^{-1} h \quad \text { in } Y .
$$

Hence, if there exists an inverse $\left(I-\lambda^{\prime} H_{\varepsilon}\right)^{-1}$, we have formula

$$
u=\left(L_{\lambda^{\prime}}+\varepsilon I\right)^{-1}=\left(I-\lambda^{\prime} H_{\varepsilon}\right)^{-1}\left(L+\left(\varepsilon+\lambda^{\prime}\right) I\right)^{-1} h .
$$

However, by assertion (5.20) it follows that

$$
\operatorname{spr}\left(\lambda^{\prime} H_{\varepsilon}\right)=\lambda^{\prime} \operatorname{spr}\left(H_{\varepsilon}\right)=\frac{\lambda^{\prime}}{\alpha(\varepsilon)}<1 \quad \text { for } \varepsilon \geq 0 .
$$

Therefore, we obtain that the inverse $\left(I-\lambda^{\prime} H_{\varepsilon}\right)^{-1}$ exists and can be represented as a C. Neumann series

$$
\left(I-\lambda^{\prime} H_{\varepsilon}\right)^{-1}=\sum_{n=0}^{\infty}\left(\lambda^{\prime} H_{\varepsilon}\right)^{n} \quad \text { for } \varepsilon \geq 0 .
$$

This implies that the operator

$$
\left(I-\lambda^{\prime} H_{\varepsilon}\right)^{-1}: Y \longrightarrow Y
$$

is bounded and strongly positive, since $H_{\varepsilon}: Y \rightarrow Y$ is strongly positive.

Summing up, we have proved that, for any $\varepsilon \geq 0$ the inverse

$$
\left(L_{\lambda^{\prime}}+\varepsilon I\right)^{-1}=\left(I-\lambda^{\prime} H_{\varepsilon}\right)^{-1} G_{\varepsilon+\lambda^{\prime}}^{N}: Y \longrightarrow Y
$$

exists, and is strongly positive and compact.

The proof of Lemma 5.3 is now complete. 
Step 2: Therefore, we can prove the following two assertions:

(a) Lemma 4.8 remains valid under condition (5.1) if we use Lemma 4.6 and Lemma 5.3.

(b) Lemma 4.9 remains valid under condition (5.1) if we use Lemmas 4.1 and 4.6 and Lemma 5.3.

In this way, we arrive at a contradiction that 0 is an $M$-simple eigenvalue of $L$. Now the proof of part (iii) of Theorem 1.2 is complete.

\section{Existence and uniqueness theorems for semilinear eigenvalue problems}

In this section we study the following general semilinear Neumann problem: Given function $f(x, \xi)$ defined on $\bar{\Omega} \times[0, \infty)$, find a positive function $u(x)$ in $\Omega$ such that

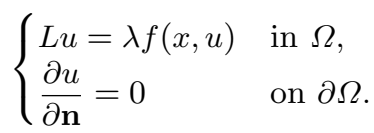

(1) In order to state our existence theorem of positive solutions of the semilinear problem (6.1), we introduce a fundamental condition (slope condition) on the nonlinear term $f(x, \xi)$ :

For a positive number $\sigma$, there exists a constant $\omega=\omega(\sigma)>0$, independent of $x \in \bar{\Omega}$, such that

$$
f(x, \xi)-f(x, \eta)>-\omega \cdot(\xi-\eta) \quad \text { for } x \in \bar{\Omega} \text { and } 0 \leq \eta<\xi \leq \sigma
$$

A non-negative function $\psi(x) \in W^{2, p}(\Omega), N<p<\infty$, is said to be a supersolution of the semilinear problem (6.1) if it satisfies the conditions

$$
\begin{cases}\mathcal{L} \psi-\lambda f(x, \psi) \geq 0 & \text { in } \Omega, \\ \frac{\partial \psi}{\partial \mathbf{n}} \geq 0 & \text { on } \partial \Omega .\end{cases}
$$

Similarly, a non-negative function $\phi(x) \in W^{2, p}(\Omega), N<p<\infty$ is said to be a subsolution of the semilinear problem (6.1) if it satisfies the conditions

$$
\begin{cases}\mathcal{L} \phi-\lambda f(x, \phi) \leq 0 & \text { in } \Omega, \\ \frac{\partial \phi}{\partial \mathbf{n}} \leq 0 & \text { on } \partial \Omega .\end{cases}
$$

Now we can state our existence theorem for the semilinear problem (6.1), which is a generalization of the sub-super-solution method due to [5, Theorem 9.4] to the VMO case:

Theorem 6.1 Assume that the function $f(x, \xi)$ belongs to $C(\bar{\Omega} \times[0, \sigma])$, and satisfies the slope condition $(R)_{\sigma}$ for some $\sigma>0$. If $\psi(x)$ and $\phi(x)$ are respectively super-and subsolutions of the semilinear problem (6.1) satisfying the condition

$$
0 \leq \phi(x) \leq \psi(x) \leq \sigma \quad \text { on } \bar{\Omega},
$$

then there exists a solution $u \in X$ of the semilinear problem (6.1) such that

$$
\phi(x) \leq u(x) \leq \psi(x) \text { on } \bar{\Omega} .
$$


Theorem 6.1 is proved in Subsection 6.1 , by using the Green operator $G_{\alpha}^{N}$ introduced in Subsection 3.3.

(2) In order to state our uniqueness theorem of positive solutions of the semilinear problem (6.1), we introduce another fundamental condition (sublinearity) on the nonlinear term $f(x, \xi)$ :

We have, for all $0<\tau<1$,

$$
f(x, \tau \xi) \geq \tau f(x, \xi), \quad x \in \bar{\Omega}, \xi>0,
$$

and

$$
f(x, 0) \geq 0, \quad x \in \bar{\Omega} .
$$

Our uniqueness theorem for the semilinear problem (6.1) is stated as follows:

Theorem 6.2 Assume that the function $f(x, \xi)$ belongs to $C(\bar{\Omega} \times[0, \sigma])$, and satisfies the slope condition $(R)_{\sigma}$ for every $\sigma>0$, and also satisfies the sublinear conditions (S1) and (S2). Then the semilinear problem (6.1) has at most one positive solution.

Theorem 6.2 is proved in Subsection 6.2, due to its length.

\subsection{Proof of Theorem 6.1}

The proof of Theorem 6.1 is divided into three steps.

Step 1: First, we consider instead of the semilinear problem (6.1) the following problem:

$$
\begin{cases}(L+(\lambda \omega) I) u=\lambda(\omega u+F(u)) & \text { in } \Omega, \\ \frac{\partial u}{\partial \mathbf{n}}=0 & \text { on } \partial \Omega,\end{cases}
$$

where $\omega>0$ is the same constant as in the slope condition $(R)_{\sigma}$, and $F(u)$ is the Nemytskii operator of $f(x, \xi)$ defined by the formula

$$
F u(x)=f(x, u(x)) \quad \text { for } x \in \bar{\Omega} .
$$

It is clear that the original semilinear problem (6.1) is equivalent to the semilinear problem (6.2).

Furthermore, since $f(x, \xi) \in C(\bar{\Omega} \times[0, \sigma])$, it is easy to verify that the semilinear problem (6.2) is equivalent to an operator equation

$$
u=\lambda(L+(\lambda \omega) I)^{-1}(\omega u+F(u)) \text { in } Y .
$$

Here

$$
G_{\lambda \omega}^{N}=(L+(\lambda \omega) I)^{-1}: C(\bar{\Omega}) \longrightarrow C^{1}(\bar{\Omega})
$$

is the Green operator $G_{\alpha}^{N}$ with $\alpha:=\lambda \omega$.

Step 2: We let

$$
H(\lambda, u):=\lambda(L+(\lambda \omega) I)^{-1}(\omega u+F(u)) \text { for } u \in C(\bar{\Omega}) .
$$

Then we have the following lemma: 
Lemma 6.1 The operator $H(\lambda, \cdot):[\phi, \psi] \rightarrow C(\bar{\Omega})$ is increasing. Here $[\phi, \psi]$ is the order interval defined by the formula

$$
[\phi, \psi]=\{u \in C(\bar{\Omega}): \phi(x) \leq u(x) \leq \psi(x) \text { on } \bar{\Omega}\} .
$$

Proof Let $u(x)$ and $v(x)$ be arbitrary functions in $C(\bar{\Omega})$ satisfying the condition

$$
\phi(x) \leq u(x) \leq v(x) \leq \psi(x) \text { on } \bar{\Omega} .
$$

Then we have the assertions

$$
\begin{aligned}
& \omega(v(x)-u(x))+(F v(x)-F u(x)) \\
= & \begin{cases}0 & \text { if } v(x)=u(x), \\
\left(\omega+\frac{F v(x)-F u(x)}{v(x)-u(x)}\right)(v(x)-u(x)) & \text { if } v(x)>u(x),\end{cases}
\end{aligned}
$$

and so, by the slope condition $(R)_{\sigma}$,

$$
\omega(v-u)+(F u-F v) \geq 0 \text { on } \bar{\Omega} .
$$

However, it follows from an application of assertion (3.6) with $\alpha:=\lambda \omega$ that the Green operator

$$
G_{\lambda \omega}^{N}=(L+(\lambda \omega) I)^{-1}: Y \longrightarrow Y
$$

is positive. Thus it follows that

$$
H(\lambda, v)-H(\lambda, u)=\lambda(L+(\lambda \omega) I)^{-1}(\omega(v-u)+(F(v)-F(u))) \geq 0 \quad \text { on } \bar{\Omega},
$$

or equivalently

$$
H(\lambda, u) \leq H(\lambda, v) \quad \text { on } \bar{\Omega} .
$$

This proves that $H(\lambda, \cdot)$ is increasing.

The proof of Lemma 6.1 is complete.

Moreover, we have the following lemma:

Lemma 6.2 The operator $H(\lambda, \cdot)$ maps the order interval $[\phi, \psi]$ into itself.

Proof Let $u(x)$ be an arbitrary function $C(\bar{\Omega})$ satisfying the condition

$$
\phi(x) \leq u(x) \leq \psi(x) \text { on } \bar{\Omega} .
$$

Then it follows from an application of Lemma 6.1 that

$$
H(\lambda, \phi) \leq H(\lambda, u) \leq H(\lambda, \psi) \quad \text { on } \bar{\Omega} .
$$

Hence, in order to prove the lemma it suffices to show that

$$
\phi \leq H(\lambda, \phi), \quad H(\lambda, \psi) \leq \psi \quad \text { on } \bar{\Omega} .
$$

If we let

$$
v:=H(\lambda, \psi)=\lambda(L+(\lambda \omega) I)^{-1}(\omega \psi+F(\psi)),
$$


then we have the assertions

$$
\begin{cases}(L+(\lambda \omega) I) v=\lambda(\omega \psi+F(\psi)) & \text { in } \Omega, \\ \frac{\partial v}{\partial \mathbf{n}}=0 & \text { on } \partial \Omega .\end{cases}
$$

However, since $\psi(x)$ is a supersolution of the semilinear problem (6.1), it follows that

$$
\begin{aligned}
& (L+(\lambda \omega) I)(v-\psi)=\lambda(\omega \psi+F(\psi))-(L+(\lambda \omega) I) \psi=-(L \psi-\lambda F(\psi)) \leq 0 \text { in } \Omega \\
& B(v-\psi)=-B \psi \leq 0 \quad \text { on } \partial \Omega
\end{aligned}
$$

Thus, by using the strong maximum principle (Theorem 3.3) we find that

$$
H(\lambda, \psi)=v \leq \psi \quad \text { on } \bar{\Omega}
$$

Indeed, by applying Theorem 3.3 with

$$
\alpha:=\lambda \omega, \quad u:=\psi-v,
$$

we obtain that

$$
\min _{\bar{\Omega}}(\psi-v) \leq 0 \Longrightarrow \psi(x) \equiv v(x) \quad \text { on } \bar{\Omega} .
$$

This implies that

$$
\psi(x)>v(x) \text { or } \psi(x) \equiv v(x) \quad \text { on } \bar{\Omega} \text {. }
$$

Similarly, we can prove that

$$
\phi \leq H(\lambda, \phi) \quad \text { on } \bar{\Omega}
$$

The proof of Lemma 6.2 is complete.

Step 3: Now we need an extension of Brouwer's fixed point theorem to the infinite-dimensional case, due to Schauder (see [7, Theorem 3.21], [13, Chapter 4], [15, p. 95, Corollary 2.3.10], [22, p. 254, Theorem 5.2.5], [44, Proposition 3.60]):

Theorem 6.3 (Schauder's fixed point theorem) A compact mapping $f$ of a closed bounded convex set $K$ in a Banach space $X$ into itself has a fixed point $x \in K$ : $f(x)=x$.

Since $H(\lambda, \cdot): C(\bar{\Omega}) \rightarrow C^{1}(\bar{\Omega})$ is compact, it follows from an application of Lemma 6.2 that the mapping $H(\lambda, \cdot):[\phi, \psi] \rightarrow[\phi, \psi]$ is compact. Furthermore, the order interval $[\phi, \psi]$ is bounded, closed and convex in the space $C(\bar{\Omega})$. Therefore, by applying Schauder's fixed point theorem we can find a function $u \in[\phi, \psi]$ such that

$$
u=H(\lambda, u)=\lambda(L+(\lambda \omega) I)^{-1}(\omega u+F(u)) \quad \text { in } Y .
$$

Now the proof of Theorem 6.1 is complete. 


\subsection{Proof of Theorem 6.2}

The proof of Theorem 6.2 is divided into three steps.

Step 1: First, the proof is essentially based on the following theorem ([5, p. 694 , Theorem 24.2]):

Theorem 6.4 Let $(E, P)$ be an ordered Banach space having the positive cone $P$ with non-empty interior $\operatorname{Int}(P)$. If $\sigma$ is a positive number, we let

$$
\bar{P}_{\sigma}=\{u \in P:\|u\| \leq \sigma\} .
$$

Assume that a mapping

$$
f: \bar{P}_{\sigma} \longrightarrow E
$$

satisfies the following two conditions:

(A) $f$ is strongly increasing, that is, if $u, v \in \bar{P}_{\sigma}$ and if $u \leq v$ and $v \neq u$, then $f(v)-f(u)$ is a point of $\operatorname{Int}(P)$.

(B) $f$ is strongly sublinear, that is, $f(0) \geq 0$ and if $u \in \bar{P}_{\sigma}$ and $u \neq 0$, then $f(\tau u)-$ $\tau f(u)$ is a point of $\operatorname{Int}(P)$ for every $0<\tau<1$.

Then the mapping $f$ has at most one positive fixed point.

Step 2: In the proof of Theorem 6.2 , we shall apply Theorem 6.4 with

$$
\begin{aligned}
& E:=Y=C(\bar{\Omega}), \\
& P:=P_{Y}=\{u \in C(\bar{\Omega}): u \geq 0 \text { on } \bar{\Omega}\}, \\
& f(\cdot):=H(\lambda, \cdot) .
\end{aligned}
$$

Substep 2-1: If $\sigma$ is a positive number, we let

$$
\left(\overline{P_{Y}}\right)_{\sigma}=\left\{u \in P_{Y}: u \leq \sigma \text { on } \bar{\Omega}\right\} .
$$

We have only to prove Theorem 6.2 in the space $\left(\overline{P_{Y}}\right)_{\sigma}$ for every $\sigma>0$. Indeed, if $u_{1}$ and $u_{2}$ are two positive solutions of problem (6.1), then we can find a constant $\sigma>0$ such that $u_{1}, u_{2} \leq \sigma$ on $\bar{\Omega}$, so that

$$
u_{1}, u_{2} \in\left(\overline{P_{Y}}\right)_{\sigma} .
$$

If we take a constant $\omega=\omega(\sigma)>0$ given in the slope condition $(R)_{\sigma}$, then we have the following lemma:

Lemma 6.3 The operator $H(\lambda, \cdot)$ maps $\left(\overline{P_{Y}}\right)_{\sigma}$ into $P_{Y}$.

Proof Let $u(x)$ be an arbitrary function in $\left(\overline{P_{Y}}\right)_{\sigma}$. Then we have, by the slope condition $(R)_{\sigma}$ with $\xi:=u$ and $\eta:=0$ and condition (S2),

$$
F(u) \geq F(0)-\omega u \geq-\omega u \text { on } \bar{\Omega},
$$

so that

$$
\omega u+F(u) \geq 0 \quad \text { on } \bar{\Omega} .
$$

Hence it follows from an application of assertion (3.6) with $\alpha:=\lambda \omega$ that

$$
H(\lambda, u)=\lambda(L+(\lambda \omega) I)^{-1}(\omega u+F(u)) \in P_{Y} .
$$

The proof of Lemma 6.3 is complete. 
Substep 2-2: Moreover, we have the following two lemmas:

Lemma 6.4 The operator $H(\lambda, \cdot):\left(\overline{P_{Y}}\right)_{\sigma} \rightarrow P_{Y}$ is strongly increasing.

Proof Lemma 6.4 follows by combining Lemma 6.1 and assertion (3.6) with $\alpha:=$ $\lambda \omega$.

Lemma 6.5 The operator $H(\lambda, \cdot):\left(\overline{P_{Y}}\right)_{\sigma} \rightarrow P_{Y}$ is strongly sublinear.

Proof Let $u(x)$ be an arbitrary function in $\left(\overline{P_{Y}}\right)_{\sigma}$ but $u(x) \neq 0$. Then we have, by the sublinear conditions $(\mathrm{S} 1)$ and $(\mathrm{S} 2)$,

$$
\begin{cases}f(x, \tau u(x)) \geq \tau f(x, u(x)) & \text { if } u(x)>0 \\ f(x, \tau u(x))=f(x, 0) \geq 0 & \text { if } u(x)=0 .\end{cases}
$$

This implies that

$$
\omega \tau u+F(\tau u)-\tau(\omega u+F(u))=F(\tau u)-\tau F(u) \geq 0 \text { and } \not \equiv 0 \quad \text { on } \bar{\Omega} .
$$

Hence it follows from an application of assertion (3.6) with $\alpha:=\lambda \omega$ that

$$
\begin{aligned}
H(\lambda, \tau u)-\tau H(\lambda, u) & =\lambda(L+(\lambda \omega) I)^{-1}(\omega \tau u+F(\tau u)-\tau(\omega u+F(u))) \\
& =\lambda G_{\lambda \omega}^{N}(\omega \tau u+F(\tau u)-\tau(\omega u+F(u))) \in \operatorname{Int}\left(P_{Y}\right) .
\end{aligned}
$$

The proof of Lemma 6.5 is complete.

Step 3: By combining Lemmas 6.3, 6.4 and 6.5, we have proved that the mapping

$$
H(\lambda, \cdot):\left(\overline{P_{Y}}\right)_{\sigma} \longrightarrow P_{Y}
$$

satisfies conditions (A) and (B) of Theorem 6.4 with $E:=Y=C(\bar{\Omega})$ and $Q:=P_{Y}$. Therefore, Theorem 6.2 follows from an application of the same theorem.

The proof of Theorem 6.2 is complete.

\section{Proof of Theorem 1.3}

In this last section, the proof of Theorem 1.3 may be carried out by using Theorems 6.1 and 6.2. The proof is divided into five steps.

Step 1: First, if we let

$$
f(x, \xi)=m(x) \xi-h(x) \xi^{2} \quad \text { for } x \in \bar{\Omega} \text { and } \xi \geq 0,
$$

then it is easy to verify that the function $f(x, \xi)$ satisfies the slope condition $(R)_{\sigma}$ for every $\sigma>0$.

Indeed, we have, for all $x \in \bar{\Omega}$ and $0 \leq \eta<\xi \leq \sigma$,

$$
\begin{aligned}
f(x, \xi)-f(x, \eta) & =m(x)(\xi-\eta)-h(x)\left(\xi^{2}-\eta^{2}\right)=(\xi-\eta)((m(x)-h(x)(\xi+\eta)) \\
& >(\xi-\eta)(m(x)-2 h(x) \xi) \\
& \geq-(\xi-\eta) \cdot \max _{\substack{x \in \bar{\Omega} \\
0 \leq \xi \leq \sigma}}(2 h(x) \xi-m(x)) .
\end{aligned}
$$


Thus, if we take a positive constant

$$
\omega=\omega(\sigma):=\max _{\substack{x \in \bar{\Omega} \\ 0 \leq \xi \leq \sigma}}(2 h(x) \xi-m(x)),
$$

then the slope condition $(R)_{\sigma}$ is satisfied.

Step 2: Secondly, we show that the function $f(x, \xi)$ satisfies the sublinear conditions (S1) and (S2).

It is clear that $f(x, 0)=0$ on $\bar{\Omega}$, which verifies condition (S2). Furthermore, since $h(x) \geq 0$ on $\bar{\Omega}$, we have, for all $x \in \bar{\Omega}, \xi>0$ and $0<\tau<1$,

$$
f(x, \tau \xi)=\tau m(x) \xi-h(x)(\tau \xi)^{2} \geq \tau\left(m(x) \xi-h(x) \xi^{2}\right)=\tau f(x, \xi)
$$

This verifies condition (S1).

Step 3: Thirdly, we assume that

$$
\int_{\Omega} m(x) \psi(x) d x<0
$$

and prove that if a pair $(\lambda, u) \in \mathbf{R} \times X$ is a positive solution of the operator equation (1.8), then it follows that (see Figure 1.2)

$$
\lambda>\lambda_{1}(m) .
$$

By rescaling, we may assume that

$$
|m(x)|<1 \text { on } \bar{\Omega} .
$$

Now assume that a pair $(\lambda, u)$ with $\lambda>0$ and $u \in P_{X} \backslash\{0\}$ satisfies the operator equation (1.8). Then, by the strong positivity of the Green operator it follows that

$$
u \in \operatorname{Int}\left(P_{Y}\right) .
$$

Indeed, if we let

$$
d:=\max _{\substack{x \in \bar{\Omega} \\ 0 \leq s \leq\|u\|_{C(\bar{\Omega})}}}|m(x)-h(x) s|+1,
$$

then we have the inequality

$$
\begin{aligned}
((\lambda d) I+L) u & =\lambda(d u+F(u)) \\
& =\lambda u(m(x)-h(x) u+d)>0 \quad \text { almost everywhere in } \Omega .
\end{aligned}
$$

Hence it follows from assertion (3.6) with $\alpha:=\lambda d$ and $f:=\lambda(d u+F(u))$ that

$$
u=\lambda((\lambda d) I+L)^{-1}(F(u)+d u) \in \operatorname{Int}\left(P_{Y}\right) .
$$

Moreover, we have, by the operator equation (1.8),

$$
\begin{aligned}
(\lambda I+L) u & =\lambda(m(x)+1) u-\lambda h(x) u^{2} \\
& <\lambda(m(x)+1) u=\lambda(M+I) u \quad \text { almost everywhere in } \Omega .
\end{aligned}
$$

By formula (4.9) with $\alpha_{0}:=\lambda$, this implies that

$$
\lambda K_{\lambda} u-u=(\lambda I+L)^{-1}(\lambda(M+I) u-(\lambda I+L) u) \in \operatorname{Int}\left(P_{Y}\right) .
$$


Therefore, the desired assertion (7.1) follows by applying Lemma 4.4 with

$$
\alpha_{0}:=\lambda, \quad w_{0}:=u, \quad \lambda:=\lambda_{1}(m) .
$$

Step 4: Finally, we prove the existence of a positive solution $u(\lambda)$ of the operator equation (1.8) for every $\lambda>\lambda_{1}(m)$, by constructing explicitly supersolutions and subsolutions to the logistic Neumann problem (1.2). It should be noticed that the uniqueness of positive solutions of the operator equation (1.8) is an immediate consequence of Theorem 6.2.

Substep 4-1: First, to construct subsolutions of the logistic Neumann problem (1.2) we consider the following Neumann eigenvalue problem:

$$
\begin{cases}(\mathcal{L}-\lambda m(x)) v=\gamma(\lambda) v & \text { in } \Omega \\ v>0 & \text { in } \Omega, \\ \frac{\partial v}{\partial \mathbf{n}}=0 & \text { on } \partial \Omega .\end{cases}
$$

Then we have the following fundamental result (cf. [45, p. 1205]):

Proposition 7.1 (i) Assume that

$$
\int_{\Omega} m(x) \psi(x) d x<0
$$

If the Neumann eigenvalue problem (7.2) has a solution $v=v(\lambda) \in P_{X} \backslash\{0\}$, then we have the assertion (see Figure 7.1)

$$
\gamma(\lambda) \begin{cases}>0 & \text { for } 0<\lambda<\lambda_{1}(m), \\ =0 & \text { for } \lambda=\lambda_{1}(m), \\ <0 & \text { for } \lambda>\lambda_{1}(m) .\end{cases}
$$

(ii) Assume that

$$
\int_{\Omega} m(x) \psi(x) d x \geq 0
$$

If the Neumann eigenvalue problem (7.2) has a solution $v=v(\lambda) \in P_{X} \backslash\{0\}$, then we have the assertion (see Figure 7.2)

$$
\gamma(\lambda)<0 \quad \text { for } \lambda>0
$$

Proof The proof is divided into three steps.

(1) It is easy to see that the Neumann eigenvalue problem (7.2) is equivalent to the problem

$$
\begin{cases}(\mathcal{L}+\lambda(1-m(x))) v(\lambda)=(\gamma(\lambda)+\lambda) v(\lambda) & \text { in } \Omega, \\ v(\lambda)>0 & \text { in } \Omega, \\ \frac{\partial v(\lambda)}{\partial \mathbf{n}}=0 & \text { on } \partial \Omega .\end{cases}
$$

However, since we have, by condition (4.7),

$$
1-m(x)>0 \text { on } \bar{\Omega},
$$




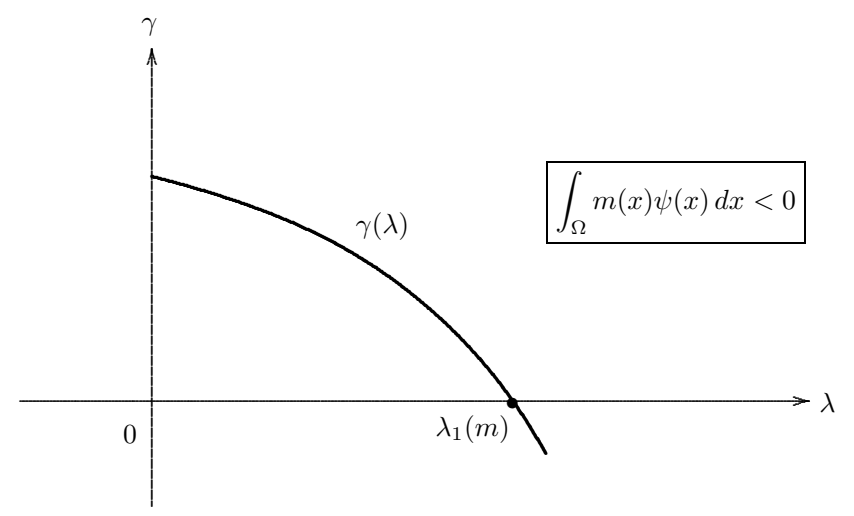

Fig. 7.1 The function $\gamma(\lambda)$ in part (i) of Proposition 7.1

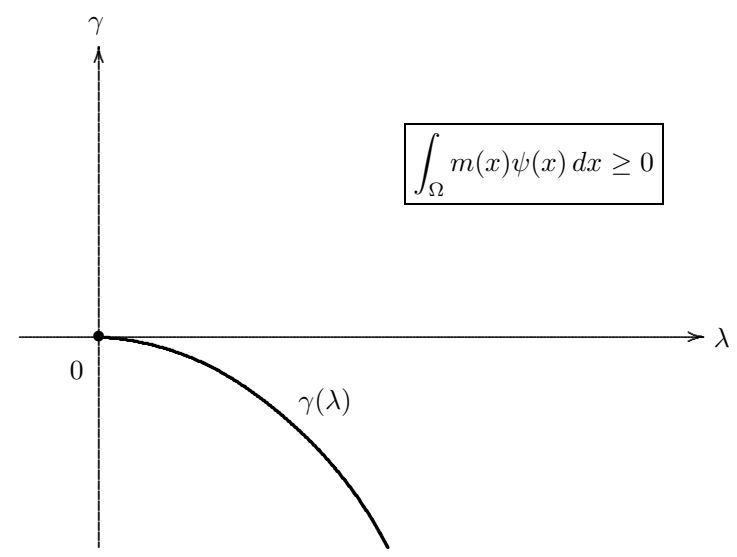

Fig. 7.2 The function $\gamma(\lambda)$ in part (ii) of Proposition 7.1

we find from the proof of assertion (3.6) that the inverse

$$
(L+\lambda(1-m(x)) I)^{-1}: Y \longrightarrow Y
$$

exists, and is strongly positive and compact. Indeed, it suffices to note that the proof of assertion (3.6) remains valid if we replace the positive constant $\alpha$ by the strictly positive function $\lambda(1-m(x))$ in $\Omega$.

Therefore, by applying the Kreĭn-Rutman theorem (Theorem 2.1) to our situation we can find a constant $\mu(\lambda)>0$ and a function $v(\lambda) \in P_{X} \backslash\{0\}$ such that

$$
(L+\lambda(1-m(x)) I) v(\lambda)=\mu(\lambda) v(\lambda) .
$$

Since we have, by formulas (7.4) and (7.5),

$$
\mu(\lambda)=\gamma(\lambda)+\lambda,
$$


then we can rewrite the operator equation (7.5) in the form

$$
\begin{cases}L v(\lambda)=\lambda\left(m(x)+\frac{\gamma(\lambda)}{\lambda}\right) v(\lambda) & \text { in } \Omega, \\ v(\lambda)>0 & \text { in } \Omega, \\ \frac{\partial v(\lambda)}{\partial \mathbf{n}}=0 & \text { on } \partial \Omega .\end{cases}
$$

Since $\lambda>0$, by applying part (i) of Theorem 1.2 with

$$
m(x):=m(x)+\frac{\gamma(\lambda)}{\lambda},
$$

we find that

$$
\begin{aligned}
& \lambda=\lambda_{1}\left(m(x)+\frac{\gamma(\lambda)}{\lambda}\right)>0, \\
& \int_{\Omega}\left(m(x)+\frac{\gamma(\lambda)}{\lambda}\right) \psi(x) d x<0 .
\end{aligned}
$$

Here it should be noticed (see Remark 5.1) that the weight function $m(x)+\frac{\gamma(\lambda)}{\lambda}$ satisfies automatically condition (M).

(2) If condition (7.2) is satisfied and if $0<\lambda<\lambda_{1}(m)$, then it follows from assertion (7.6) that

$$
0<\lambda=\lambda_{1}\left(m(x)+\frac{\gamma(\lambda)}{\lambda}\right)<\lambda_{1}(m)
$$

Therefore, by applying Proposition 5.1 with

$$
\begin{aligned}
& m_{1}(x):=m(x)+\frac{\gamma(\lambda)}{\lambda}, \\
& m_{2}(x):=m(x)
\end{aligned}
$$

we find that

$$
\gamma(\lambda)>0 \quad \text { for } 0<\lambda<\lambda_{1}(m)
$$

Secondly, if $\lambda=\lambda_{1}(m)$, then we obtain from part (i) of Theorem 1.2 that the operator equation (1.5) admits a positive eigenfunction $v \in P_{X} \backslash\{0\}$, that is,

$$
\begin{cases}\left(L-\lambda_{1}(m) m(x)\right) v(\lambda)=0 \cdot v(\lambda) & \text { in } \Omega, \\ v(\lambda)>0 & \text { in } \Omega, \\ \frac{\partial v(\lambda)}{\partial \mathbf{n}}=0 & \text { on } \partial \Omega .\end{cases}
$$

This implies that $\gamma\left(\lambda_{1}(m)\right)=0$.

Finally, if $\lambda>\lambda_{1}(m)$, it follows from assertion (7.7) that

$$
\lambda_{1}\left(m(x)+\frac{\gamma(\lambda)}{\lambda}\right)=\lambda>\lambda_{1}(m) .
$$

By applying Proposition 5.1 with

$$
m_{1}(x):=m(x)
$$




$$
m_{2}(x):=m(x)+\frac{\gamma(\lambda)}{\lambda}
$$

we find that

$$
\gamma(\lambda)<0 \text { for } \lambda>\lambda_{1}(m) .
$$

(3) If condition (7.3) is satisfied, then it follows from assertion (7.7) that

$$
\int_{\Omega} m(x) \psi(x) d x+\frac{\gamma(\lambda)}{\lambda}\left(\int_{\Omega} \psi(x) d x\right)=\int_{\Omega}\left(m(x)+\frac{\gamma(\lambda)}{\lambda}\right) \psi(x) d x<0,
$$

so that

$$
\gamma(\lambda)<-\lambda \frac{\int_{\Omega} m(x) \psi(x) d x}{\int_{\Omega} \psi(x) d x} \leq 0
$$

This proves that

$$
\gamma(\lambda)<0 \quad \text { for } \lambda>0
$$

The proof of Proposition 7.1 is complete.

Substep 4-2: In the case where

$$
\int_{\Omega} m(x) \psi(x) d x<0
$$

it follows from an application of part (i) of Proposition 7.1 that, for every $\lambda>$ $\lambda_{1}(m)$ we can find a constant $\gamma(\lambda)<0$ and a positive function $v(\lambda) \in P_{X} \backslash\{0\}$ such that

$$
(L-\lambda m(x)) v(\lambda)=\gamma(\lambda) v(\lambda) .
$$

Then we obtain that the function $\varepsilon v(\lambda)$ is a subsolution of the logistic Neumann problem (1.2) if $\varepsilon=\varepsilon(\lambda)$ is so small that

$$
0<\varepsilon \leq-\left(\frac{\gamma(\lambda)}{\lambda}\right) \frac{1}{\max _{\bar{\Omega}} h \cdot \max _{\bar{\Omega}} v(\lambda)} .
$$

Indeed, we have the assertions

$$
\begin{aligned}
L(\varepsilon v(\lambda))-\lambda m(x)(\varepsilon v(\lambda))+\lambda h(x)(\varepsilon v(\lambda))^{2} & =\varepsilon v(\lambda)(\gamma(\lambda)+\lambda \varepsilon h(x) v(\lambda)) \\
& \leq 0 \quad \text { in } \Omega
\end{aligned}
$$

and

$$
\frac{\partial}{\partial \mathbf{n}}(\varepsilon v(\lambda))=\varepsilon \frac{\partial v(\lambda)}{\partial \mathbf{n}}=0 \quad \text { on } \partial \Omega
$$

Furthermore, it follows that the constant function

$$
w(x) \equiv \ell:=\frac{\max _{\bar{\Omega}} m}{\min \bar{\Omega} h}
$$

is a supersolution of the logistic Neumann problem (1.2) for all $\lambda>0$, since we have the assertions

$$
\begin{aligned}
L w-\lambda m(x) w+\lambda h(x) w^{2} & =-\lambda m(x) \ell+\lambda h(x) \ell^{2}=\lambda \ell(h(x) \ell-m(x)) \\
& \geq 0 \quad \text { in } \Omega
\end{aligned}
$$


and

$$
\frac{\partial w}{\partial \mathbf{n}}=0 \quad \text { on } \partial \Omega .
$$

Therefore, by applying Theorem 6.1 (the sub-super-solution method) to our situation we can find a positive solution $u(\lambda) \in P_{X} \backslash\{0\}$ of the operator equation (1.8) such that

$$
0<\varepsilon(\lambda) v(\lambda) \leq u(\lambda) \leq \ell \quad \text { in } \Omega .
$$

This proves that the operator equation (1.8) has a unique positive solution $u(\lambda) \in$ Int $\left(P_{Y}\right)$ for every $\lambda>\lambda_{1}(m)$ with the desired uniform estimate (1.11) as in Figure 1.5 .

Step 5: In the case where

$$
\int_{\Omega} m(x) \psi(x) d x \geq 0
$$

the proof of Theorem 1.3 may be carried out, just as in the case $\int_{\Omega} m(x) \psi(x) d x<$ 0 , if we make use of part (ii) of Proposition 7.1.

Indeed, since we have the assertion

$$
\gamma(\lambda)<0 \text { for } \lambda>0 \text { under condition (7.5), }
$$

we can prove that the operator equation (1.8) has a unique positive solution $u(\lambda) \in$ Int $\left(P_{Y}\right)$ for every $\lambda>0$ just as in the proof of Step 4 .

Substep 5-1: If $\int_{\Omega} m(x) \psi(x) d x>0$, then we can show that there is a secondary bifurcation from the line $\{0\} \times \mathbf{R}$ of trivial solutions (see Figure 1.3).

To do this, it suffices to prove the following lemma (cf. [27, Example 28.6]):

Lemma 7.1 Assume that

$$
\int_{\Omega} m(x) \psi(x) d x>0 .
$$

Then the solution $u(\lambda)$ converges to the constant

$$
c=\frac{\int_{\Omega} m(x) \psi(x) d x}{\int_{\Omega} h(x) \psi(x) d x}
$$

in $C(\bar{\Omega})$ as $\lambda \downarrow 0$.

Proof Since we have the uniform estimate (1.11) for all $\lambda>0$, it follows that

$$
\begin{aligned}
\|L u(\lambda)\|_{C(\bar{\Omega})} & =\lambda\left\|m(x) u(\lambda)-h(x) u(\lambda)^{2}\right\|_{C(\bar{\Omega})} \\
& \leq \lambda\left(\ell\|m\|_{C(\bar{\Omega})}+\ell^{2}\|h\|_{C(\bar{\Omega})}\right) .
\end{aligned}
$$

This implies that

$$
\begin{aligned}
\|u(\lambda)\|_{X} & =\|u(\lambda)\|_{C(\bar{\Omega})}+\|L u(\lambda)\|_{C(\bar{\Omega})} \\
& \leq \ell\left(1+\|m\|_{C(\bar{\Omega})}\right)+\ell^{2}\|h\|_{C(\bar{\Omega})} \quad \text { for } 0<\lambda<1 .
\end{aligned}
$$

Therefore, by the compactness argument we can choose a sequence $\left(\lambda_{j}, u\left(\lambda_{j}\right)\right)$, with $0<\lambda_{j}<1$ and $u\left(\lambda_{j}\right) \in \operatorname{Int}\left(P_{Y}\right)$, and a function $v \in Y=C(\bar{\Omega})$ such that

$$
L u\left(\lambda_{j}\right)=\lambda_{j}\left(m(x) u\left(\lambda_{j}\right)-h(x) u\left(\lambda_{j}\right)^{2}\right),
$$




$$
\begin{aligned}
& \lambda_{j} \rightarrow 0, \\
& u\left(\lambda_{j}\right) \longrightarrow v \quad \text { in } C(\bar{\Omega}) .
\end{aligned}
$$

Hence we have, by assertions (7.8),

$$
\left\{\begin{array}{l}
L u\left(\lambda_{j}\right) \longrightarrow 0 \quad \text { in } C(\bar{\Omega}), \\
u\left(\lambda_{j}\right) \longrightarrow v \quad \text { in } C(\bar{\Omega}),
\end{array}\right.
$$

and so, by the closedness of $L$,

$$
\left\{\begin{array}{l}
v \in \mathcal{D}(L), \\
L v=0 .
\end{array}\right.
$$

This proves that we have, for some constant $c \geq 0$,

$$
v(x) \equiv c \quad \text { in } \Omega,
$$

since $u\left(\lambda_{j}\right) \in \operatorname{Int}\left(P_{Y}\right)$ and $\mathcal{N}(L)=$ span [1]. However, it follows from an application of Lemma 4.9 with $m(x):=-m(x)$ that zero is an $M$-simple eigenvalue of $L$. Hence, by applying Theorem 2.2 due to Crandall-Rabinowitz [19] with

$$
\begin{aligned}
& X=Y:=C(\bar{\Omega}), \\
& F(\lambda, u):=L u-\lambda\left(m(x) u-h(x) u^{2}\right), \\
& F_{u}(0,0):=L, \quad F_{\lambda u}(0,0):=-M, \\
& x_{0}:=1,
\end{aligned}
$$

we conclude that $c=0$ is excluded. Indeed, it suffices to note that there are two lines $\mathbf{R} \times\{0\}$ and $\{0\} \times \mathbf{R}$ of trivial solutions bifurcating at the point $(0,0)$.

Finally, it remains to prove that the positive constant $c$ is given by formula (1.10). Since $\psi \in \mathcal{N}\left(L^{*}\right)$, it follows from formula (7.8a) that

$$
\begin{aligned}
& \lambda_{j}\left(\int_{\Omega} m(x) u\left(\lambda_{j}\right) \psi(x) d x-\int_{\Omega} h(x) u\left(\lambda_{j}\right)^{2} \psi(x) d x\right) \\
= & \int_{\Omega} L u\left(\lambda_{j}\right) \cdot \psi(x) d x=\int_{\Omega} u\left(\lambda_{j}\right) \cdot L^{*} \psi d x \\
= & 0 \quad \text { for } 0<\lambda_{j}<1,
\end{aligned}
$$

so that

$$
\int_{\Omega} m(x) u\left(\lambda_{j}\right) \psi(x) d x=\int_{\Omega} h(x) u\left(\lambda_{j}\right)^{2} \psi(x) d x .
$$

Therefore, by passing to the limit $j \rightarrow \infty$ in formula (7.10) we obtain from assertions (7.8c) and (7.9) that

$$
c \int_{\Omega} m(x) \psi(x) d x=c^{2} \int_{\Omega} h(x) \psi(x) d x .
$$

This proves the desired formula (1.10), since $c>0$.

The proof of Lemma 7.1 is complete.

Substep 5-2: If $\int_{\Omega} m(x) \psi(x) d x=0$, by arguing as in the proof of Lemma 7.1 we can prove the following lemma: 
Lemma 7.2 Assume that

$$
\int_{\Omega} m(x) \psi(x) d x=0
$$

Then the solution $u(\lambda)$ converges to zero in $C(\bar{\Omega})$ as $\lambda \downarrow 0$.

Proof Indeed, it suffices to note that formula (7.11) implies $c=0$, since we have the inequality

$$
\int_{\Omega} h(x) \psi(x) d x \geq \min _{\bar{\Omega}} h \cdot \int_{\Omega} \psi(x) d x>0 .
$$

The proof of Lemma 7.2 is complete.

By virtue of Lemma 7.2, we obtain that there is a positive solution curve $\{(\lambda, u(\lambda)): \lambda>0\}$ bifurcating at the point $(0,0)$ from the line $\mathbf{R} \times\{0\}$ of trivial solutions, as is shown in Figure 1.4. Indeed, we may let $c:=0$ in Figure 1.3.

Now the proof of Theorem 1.3 is complete.

Acknowledgements I am grateful to the referees for their valuable suggestions and for informing me of additional references, which improved the presentation of this paper.

\section{References}

1. Acquistapace, P.: On BMO regularity for linear elliptic systems. Ann. Mat. Pura Appl. 161, 231-269 (1992)

2. Adams, R. A., Fournier, J. J. F.: Sobolev spaces, second edition. Pure and Applied Mathematics, vol. 140, Elsevier/Academic Press, Amsterdam (2003)

3. Afrouzi, G. A., Brown, K. J.: On principal eigenvalues for boundary value problems with indefinite weight and Robin boundary conditions. Proc. Amer. Math. Soc. 127, 125-130 (1999)

4. Agmon, S., Douglis, A., Nirenberg, L.: Estimates near the boundary for solutions of elliptic partial differential equations satisfying general boundary conditions I. Comm. Pure Appl. Math. 12, 623-727 (1959)

5. Amann, H.: Fixed point equations and nonlinear eigenvalue problems in ordered Banach spaces. SIAM Rev. 18, 620-709 (1976)

6. Amann, H.: Nonlinear elliptic equations with nonlinear boundary conditions. In: New developments in differential equations, W. Eckhaus (ed.), pp. 43-63. North-Holland Mathematics Studies, vol. 21, North-Holland, Amsterdam New York Oxford (1976)

7. Ambrosetti, A., Malchiodi, A.: Nonlinear analysis and semilinear elliptic problems. Cambridge Studies in Advanced Mathematics, no. 104, Cambridge University Press, Cambridge (2007)

8. Ambrosetti, A., Prodi, G.: A primer of nonlinear analysis, paperback edition (with corrections). Cambridge Studies in Advanced Mathematics, No. 34, Cambridge University Press, Cambridge (1995)

9. Bergh, J., Löfström, J.: Interpolation spaces, an introduction. Springer-Verlag, Berlin Heidelberg New York (1976)

10. Bony, J.-M.: Principe du maximum dans les espaces de Sobolev. C. R. Acad. Sc. Paris Sér. A-B 265, A333-A336 (1967)

11. Bramanti, M., Cerutti, M. C.: $W_{p}^{1,2}$ Solvability for the Cauchy-Dirichlet problem for parabolic equations with VMO coefficients. Comm. Partial Differential Equations 18, 1735-1763 (1993)

12. Brown, K. J., Lin, S. S.: On the existence of positive eigenfunctions for an eigenvalue problem with indefinite weight function. J. Math. Anal. Appl. 75, 112-120 (1980)

13. Brown, R. F.: A topological introduction to nonlinear analysis, third edition. Springer, Cham (2014) 
14. Calderón, A. P., Zygmund, A.: On the existence of certain singular integrals. Acta Math. 88, 85-139 (1952)

15. Chang, K.-C.: Methods in nonlinear analysis. Springer Monogr. Math., Springer-Verlag, Berlin (2005)

16. Chiarenza, F., Frasca, M., Longo, P.: Interior $W^{2, p}$ estimates for nondivergence elliptic equations with discontinuous coefficients. Ricerche Mat. 60, 149-168 (1991)

17. Chiarenza, F., Frasca, M., Longo, P.: $W^{2, p}$-solvability of the Dirichlet problem for nondivergence elliptic equations with VMO coefficients. Trans. Amer. Math. Soc. 336, 841-853 (1993)

18. Chow, S. N., Hale, J. K.: Methods of bifurcation theory. Springer-Verlag, New York Heidelberg Berlin (1982)

19. Crandall, M. G., Rabinowitz, P. H.: Bifurcation from simple eigenvalues. J. Functional Analysis 8, 321-340 (1971)

20. Crandall, M. G., Rabinowitz, P. H.: Bifurcation, perturbation of simple eigenvalues, and linearized stability. Arch. Rat. Mech. Anal. 52, 161-180 (1973)

21. Di Fazio, G., Palagachev, D. K.: Oblique derivative problem for elliptic equations in nondivergence form with VMO coefficients. Comment. Math. Univ. Carolin. 37, 537-556 (1996)

22. Drábek, P., Milota, J.: Methods of nonlinear analysis, Applications to differential equations, second edition. Birkhäuser Advanced Texts: Basler Lehrbücher. Birkhäuser/Springer Basel AG, Basel (2013)

23. Friedman, A.: Partial differential equations. Dover Publications Inc., Mineola, New York $(1969 / 2008)$

24. Garnett, J. B.: Bounded analytic functions. Academic Press, New York London Toronto Sydney San Francisco (1981)

25. Gilbarg, D., Trudinger, N. S.: Elliptic partial differential equations of second order, reprint of the 1998 edition. Classics in Mathematics, Springer-Verlag, New York Berlin Heidelberg Tokyo (1998)

26. Gohberg, I. C., Kreı̆n, M. G.: The basic propositions on defect numbers, root numbers and indices of linear operators (Russian). Uspehi Mat. Nauk., 12, 43-118 (1957). English translation: Amer. Math. Soc. Transl. (2) 13, 185-264 (1960)

27. Hess, P.: Periodic-parabolic boundary value problems and positivity. Pitman Research Notes in Mathematical Series 247, Longman Scientific \& Technical, Harlow, Essex (1991)

28. Hess, P., Kato, T.: On some linear and nonlinear eigenvalue problems with an indefinite weight function. Comm. Partial Differential Equations 5, 999-1030 (1980)

29. John, F., Nirenberg, L.: On functions of bounded mean oscillation. Comm. Pure and Appl. Math. 14, 175-188 (1961)

30. Kato, T.: Schrödinger operators with singular potentials. Israel J. Math. 13, 135-148 (1972)

31. Kato, T.: Perturbation theory for linear operators, 1980 edition. Classics in Mathematics, Springer-Verlag, Berlin Heidelberg New York (1980)

32. Kreĭn, M. G., Rutman, M. A.: Linear operators leaving invariant a cone in a Banach space. Amer. Math. Soc. Transl. 10, 199-325 (1962)

33. Lieberman, G. M.: Local estimates for subsolutions and supersolutions of oblique derivative problems for general second order elliptic equations. Trans. Amer. Math. Soc. 304, 343353 (1987)

34. Maugeri, A., Palagachev, D. K.: Boundary value problem with an oblique derivative for uniformly elliptic operators with discontinuous coefficients. Forum Math. 10, 393-405 (1998)

35. Maugeri, A., Palagachev, D. K., Softova, L. G.: Elliptic and parabolic equations with discontinuous coefficients. Mathematical Research, vol. 109, Wiley-VCH, Berlin (2000)

36. Meyers, N.: An $L^{p}$-estimate for the gradient of solutions of second order elliptic divergence equations. Ann. Scuola Norm. Sup. Pisa 17, 189-206 (1963)

37. Miranda, C.: Sulle equazioni ellittiche del secondo ordine di tipo non variazionale a coefficienti discontinui. Ann. Mat. Pura Appl. 63, 353-386 (1963)

38. Nirenberg, L.: Topics in nonlinear functional analysis, revised reprint of the 1974 original. Courant Lecture Notes in Mathematics, no, 6, New York University, Courant Institute of Mathematical Sciences, New York. American Mathematical Society, Providence, Rhode Island (2001)

39. Ragusa, M. A.: Elliptic boundary value problem in Vanishing Mean Oscillation hypothesis. Comment. Math. Univ. Carolin. 40, 651-663 (1999) 
40. Ragusa, M. A., Scapellato, A.: Mixed Morrey spaces and their applications to partial differential equations. Nonlinear Anal. TMA 151, 51-65 (2017)

41. Sarason, D.: Functions of vanishing mean oscillation. Trans. Amer. Math. Soc. 207, 391$405(1975)$

42. Saut, J. C., Scheurer, B.: Remarks on a non linear equation arising in population genetics. Comm. Partial Differential Equations 3, 907-931 (1978)

43. Schechter, M.: Principles of functional analysis, second edition. Graduate Studies in Mathematics, vol. 36, American Mathematical Society, Providence, Rhode Island (2002)

44. Schwartz, J. T.: Nonlinear functional analysis. Gordon and Breach, New York (1969)

45. Senn, S.: On a nonlinear elliptic eigenvalue problem with Neumann boundary conditions, with an application to population genetics. Comm. Partial Differential Equations 8, 11991228 (1983)

46. Senn, S., Hess, P.: On positive solutions of a linear elliptic eigenvalue problem with Neumann boundary conditions. Math. Ann. 258, 459-470 (1982)

47. Taira, K.: Boundary value problems for elliptic integro-differential operators. Math. Z. 222, 305-327 (1996)

48. Taira, K.: Introduction to semilinear elliptic boundary value problems. Taiwanese J. Math 2, 127-172 (1998)

49. Taira, K.: Introduction to diffusive logistic equations in population dynamics. Korean J. Comput. Appl. Math. 9, 289-347 (2002)

50. Taira, K.: Logistic Dirichlet problems with discontinuous coefficients. J. Math. Pures Appl. 82, $1137-1190(2003)$

51. Taira, K.: Diffusive logistic equations with degenerate boundary conditions. Mediterr. J. Math. 1, 315-365 (2004)

52. Taira, K.: Degenerate elliptic eigenvalue problems with indefinite weights. Mediterr. J. Math. 5, 133-162 (2008)

53. Taira, K.: Semigroups, boundary value problems and Markov processes, second edition Springer Monographs in Mathematics series, Springer-Verlag, Berlin Heidelberg New York (2014)

54. Taira, K.: Analytic semigroups and semilinear initial-boundary value problems, second edition. London Mathematical Society Lecture Note Series, vol. 434, Cambridge University Press, Cambridge (2016)

55. Taira, K.: Boundary value problems and Markov processes: Functional analysis methods for Markov processes, third edition. Lecture Notes in Mathematics, no. 1499, SpringerVerlag, Berlin Heidelberg New York (2020)

56. Taira, K.: Oblique derivative problems and Feller semigroups with discontinuous coefficients. Ricerche Mat. https://doi.org/10.1007/s11587-020-00509-5

57. Talenti, G.: Equazioni lineari ellittiche in due variabili. Matematiche (Catania) 21, 339$376(1966)$

58. Tanabe, H.: Functional analytic methods for partial differential equations. Marcel Dekker, New York Basel (1997)

59. Torchinsky, A.: Real-variable methods in harmonic analysis. Dover Books on Mathematics, Dover Publications Inc., Mineola, New York (1986/2004)

60. Triebel, H.: Theory of function spaces. Monographs in Mathematics, vol. 78. BirkhäuserVerlag, Basel Boston Stuttgart (1983)

61. Troianiello, G. M.: Elliptic differential equations and obstacle problems. Plenum Press, New York (1987)

62. Yosida, K.: Functional analysis, reprint of the sixth (1980) edition. Classics in Mathematics, Springer-Verlag, Berlin Heidelberg New York (1980) 